\title{
Development of trigger software for the Silicon and Fibre Trackers and a study of $B$ meson lifetimes for the $\mathrm{D} \emptyset$ experiment
}

\author{
Robert Arthur Illingworth \\ Imperial College of Science, Technology and Medicine
}

A thesis submitted for the degree of

Doctor of Philosophy

of The University of London

and the Diploma of Imperial College.

January, 2002 


\title{
Development of trigger software for the Silicon and Fibre Trackers and a study of $B$ meson lifetimes for the $\mathrm{D} \varnothing$ experiment
}

\author{
Robert Arthur Illingworth \\ Imperial College of Science, Technology and Medicine
}

January, 2002

\begin{abstract}
The $\mathrm{D} \varnothing$ detector has recently undergone a major upgrade to maximise its potential to fully exploit Run II at the Tevatron $2 \mathrm{TeV}$ proton-antiproton collider. The upgrade includes a completely new central tracking system with an outer scintillating fibre tracker and an inner silicon vertex detector. This thesis describes the development of the software to 'unpack' the raw data from the central tracking detectors into a useful form, and the development of the Level 3 trigger algorithms to cluster the hit information from these detectors.

One of the many areas of physics that is being studied by the $D \varnothing$ experiment is the physics of $B$ mesons, particularly that involving $\mathrm{CP}$ violation. The second part of the thesis details a constrained mass fitting tool written to aid the reconstruction of $B$ particles, and a Monte Carlo study into measuring the lifetime of $B^{+}$and $B^{0}$ mesons.

This thesis lays the foundations for the means by which physics is extracted from the vast amount of Tevatron data - the trigger - and illustrates how analyses will proceed through the key reconstruction of heavy quarks.
\end{abstract}




\section{Acknowlegements}

I would like to thank...

My supervisor, John Hassard, and Trevor Bacon, Gavin Davies, and Vivek Jain, who read some or all of this and pointed out many mistakes, incomprehensible sentences, and general inanities.

Everybody at Imperial and at Fermilab who provided help, answered my stupid questions, or supplied encouragement, support, or future employment, including: Ela Barberis, Daniela Bauer, Ray Beuselinck, Freya Bleckman, Amber Boehnlein, Gustaaf Brooijmans, Dave Colling, Marcel Demarteau, Harald Fox, Jonathan Hays, Mike Hildreth, Rick Jesik, Slava Kulik, Aurelio Juste, Meena Narain, Michele Petteni, Olivia Vizcarra and all the others who I've missed off this list.

PPARC for their financial support and Peter Dornan for accepting me into the Imperial College HEP group.

And Louise, Barry, Elaine, Alex, Rod, Richard and a few others for far too much alcohol filled, thesis delaying, time. 


\section{Contents}

$\begin{array}{ll}\text { Abstract } & 2\end{array}$

$\begin{array}{ll}\text { Acknowlegements } & 3\end{array}$

$\begin{array}{ll}\text { Contents } & 4\end{array}$

$\begin{array}{ll}\text { List of Figures } & 10\end{array}$

List of Tables

$\begin{array}{lll}\text { Chapter 1. Introduction } & 16\end{array}$

1.1 The Standard Model and beyond 16

$\begin{array}{lll}1.2 & \text { The Tevatron upgrade } & 17\end{array}$

$\begin{array}{lll}1.3 & \text { The D } \varnothing \text { upgrade } & 19\end{array}$

1.4 The scope of this thesis 20

$\begin{array}{ll}\text { 1.4.1 Triggering and reconstruction software } & 20\end{array}$

$\begin{array}{lll}\text { 1.4.2 } & B \text { physics and CP violation } & 20\end{array}$ 
Chapter 2. CP violation, $B$ mesons, and the CKM model

2.1 Introduction to $\mathrm{CP}$ violation

2.1.1 CP violation in neutral kaons

2.2 Discrete symmetries

2.2.1 C and P symmetry

2.2.2 T symmetry

2.3 The CPT theorem

2.4 CP violation in the Standard Model

$2.5 \mathrm{CP}$ violating processes

2.5.1 CP violation in mixing 30

2.5.2 CP violation in the decay 31

2.5.3 CP violation in the interference of mixing and decay 32

$\begin{array}{lll}2.6 & B \text { meson decays } & 33\end{array}$

2.6.1 Production at the Tevatron 33

2.6.2 Lifetimes 33

2.6.3 CP violation in the $B$ meson system 35

$\begin{array}{lll}2.7 & \text { Experimental results } & 37\end{array}$

$\begin{array}{lll}\text { 2.7.1 Past and current and future experiments } & 38\end{array}$

$\begin{array}{lll}2.7 .2 & \text { Current results } & 39\end{array}$

2.7.3 CP violation measurements with the $\mathrm{D} \varnothing$ detector 40 
3.1 Overview of the upgraded detector

3.2 Central tracking

3.2.1 Solenoid

3.2.2 Silicon Microstrip Tracker

3.2.3 Central fibre tracker

3.2.4 Preshower detectors

3.3 Calorimeter

3.3.1 Central calorimeter

3.3.2 End calorimeter

3.3.3 Intercryostat detector

3.4 Muon system

3.4.1 Wide angle muon system

3.4.2 Forward muon system 
Chapter 4. Silicon Microstrip Tracker readout software

4.1 SMT electronics and readout

4.2 Raw data format

4.2.1 VRB header

4.2.2 HDI data

4.3 Unpacking software design

4.3.1 Unpacking

4.3.2 Offline unpacking

4.3.3 Level 3 unpacking

4.3.4 Packing

4.4 Clustering in the Level 3 trigger

4.4.1 SMT Coordinate systems

4.4.2 Two dimensional clustering

4.4.3 Three dimensional clustering

4.5 Results from data

4.5.1 Results from the cosmic ray test

4.5.2 Results from first collisions

5.1 CFT electronics and readout $\quad 93$ 
5.1.2 CFT raw data format

5.2 Unpacking software

5.2.1 Offline unpacking

5.2.2 Level 3 trigger unpacking

5.3 Level 3 trigger clustering

5.3.1 Results

7.1 Introduction

7.2 Monte Carlo data sample 
7.8.1 Two dimensional lifetime distribution

7.8.2 Three dimensional lifetime distribution

134

7.8.3 Likelihood fit to lifetime

Chapter 8. Conclusions

8.1 $B$ physics and CP violation measurements at $\mathrm{D} \varnothing$

8.2 Measuring $\sin 2 \beta$

8.2.1 Flavour tagging

$8.2 .2 \sin 2 \beta$

8.3 Other $B$ and CP measurements at D $\varnothing$

8.3.1 $B_{s}$ mixing

8.3.2 $\quad B_{d}^{0} \rightarrow \pi^{+} \pi^{-}, B_{s}^{0} \rightarrow K^{+} K^{-}$

8.3.3 $\quad B_{s} \rightarrow J / \psi \phi$

8.3.4 Other $B$ measurements 


\section{List of Figures}

2.1 The CKM unitarity triangle in the Wolfenstein parameterization

2.2 Box diagrams for $\bar{K}^{0} \rightarrow K^{0}$ mixing via top quarks

2.3 Leading order diagrams for $b \bar{b}$ production

2.4 Spectator model of $B$ decay

2.5 Non-spectator $B$ decays

2.6 Tree and penguin diagrams for $B^{0} \rightarrow \pi^{+} \pi^{-}$

2.7 Global fit for $(\bar{\rho}, \bar{\eta})$

3.1 Side view of the upgraded detector

3.2 The tracking system

3.3 The Silicon Microstrip Tracker (SMT) 46

3.4 The forward preshower detector

3.5 The calorimeter 
4.3 Flowchart for the SMT unpacking algorithm

4.4 The class design of the SmtRawUnp2Data package

4.5 Key for class diagrams

4.6 The class design of the Level 3 SMT unpacking and clustering tool

4.7 SVX chip pedestals

4.8 The class design of the SmtData2RawUnp package

4.9 The local coordinate system for ladders and wedges.

4.10 Calculation of hit position

4.11 The global SMT coordinate system

4.12 Monte Carlo spatial resolution of the axial barrel clusters

4.13 Monte Carlo efficiency and misidentification of axial clusters

4.14 Monte Carlo spatial resolution in $z$ of $90^{\circ}$ stereo barrel clusters

4.15 Monte Carlo spatial resolution of F disks

4.16 Monte Carlo spatial resolution of $\mathrm{H}$ disks

4.17 Monte Carlo spatial resolution in $z$ of $2^{\circ}$ stereo barrel clusters

4.18 A track from the cosmic ray test

4.19 Comparison of Level 3 and offline cosmic ray hit positions

4.21 SMT unpacking and clustering time (barrels only) for different Monte Carlo event types

4.22 The unpacking and clustering time (barrels only) for $Z \rightarrow \mu \mu$ events with varying numbers of added minimum bias interactions. 
4.23 The unpacking and clustering time (barrels only) for $Z \rightarrow \mu \mu$ events with varying added noise level

4.24 Level 3 SMT unpacking and 2D clustering time for real data (barrels only) 92

5.1 Diagram of the CFT readout system

5.2 Flowchart for the CFT offline unpacking algorithm

5.3 The class design of the Level 3 CFT unpacking and clustering tool

5.4 Monte Carlo $t \bar{t}$ event reconstructed with the Level 3 CFT tracker

5.5 CFT track - cluster residuals for axial clusters

5.6 Event display from running the CFT unpacking, clustering, and tracking on early data (taken 1 December 2001) with the full axial CFT instrumented and being read out

5.7 CFT unpacking and clustering time for different Monte Carlo event types.106

5.8 The unpacking and clustering time for $Z \rightarrow \mu \mu$ events with varying numbers of added minimum bias interactions.

5.9 The unpacking and clustering time for $Z \rightarrow \mu \mu$ events with varying levels of added noise

$6.1 x$-momentum residuals $\left(p_{\text {reconstructed }}-p_{\text {generated }}\right)$ for reconstructed Monte Carlo $J / \psi$

$6.2 y$-momentum residuals $\left(p_{\text {reconstructed }}-p_{\text {generated }}\right)$ for reconstructed Monte Carlo $J / \psi$

$6.3 z$-momentum residuals $\left(p_{\text {reconstructed }}-p_{\text {generated }}\right)$ for reconstructed Monte Carlo $J / \psi$ 
6.4 Momentum pulls $\left(p_{\text {reconstructed }}-p_{\text {generated }}\right) / \sigma_{p}$ for vertex fitted Monte Carlo $J / \psi$

6.5 Momentum pulls $\left(p_{\text {reconstructed }}-p_{\text {generated }}\right) / \sigma_{p}$ for vertex fitted, mass constrained Monte Carlo $J / \psi$

7.1 $p_{\mathrm{T}}$ spectrum of the muons and kaons from the $B^{+}$Monte Carlo sample

$7.2 \mathrm{~J} / \psi$ invariant mass

7.3 Mass of the $J / \psi$ s used to make $B^{+}$'s

$7.4 \mathrm{~J} / \psi$ invariant mass using the muon detectors from real data

7.5 $\chi^{2}$ distribution of constrained vertex fit to form $B^{+}$candidates

7.6 Reconstructed invariant mass of all $B^{+}$candidates (Monte Carlo)

7.7 Reconstructed $B^{+}$invariant mass

7.8 Relationship between the actual and reconstructed vertices and the $B^{+}$ momentum

7.9 Bias on primary vertex position

7.10 Primary vertex resolution

7.11 Secondary vertex resolution

7.12 Reconstructed $B^{+}$mass from combined signal and background samples

7.13 Reconstructed $B^{+}$proper decay length from the combined signal and background, and from direct $J / \psi$ production alone

7.14 2D proper decay length for $B^{+}$

7.15 Difference between reconstructed $B^{+}$2D proper decay length and Monte Carlo proper decay length 
7.17 Difference between reconstructed $B^{+}$2D proper decay length and Monte Carlo proper decay length, divided by the error on the reconstructed length.

$7.183 \mathrm{D}$ proper decay length for $B^{+}\left(B^{+} \rightarrow J / \psi K^{+}\right.$events only $)$

7.19 Difference between reconstructed $B^{+} 3 \mathrm{D}$ proper decay length and Monte Carlo proper decay length

7.20 The errors on the 3D proper decay length

7.21 Difference between reconstructed $B^{+}$proper decay length and Monte Carlo 3D proper decay length, divided by the error on the reconstructed length.

7.22 Background and signal fits to the transverse proper decay length

7.23 Background and signal fits to the transverse proper decay length 


\section{List of Tables}

1.1 Tevatron parameters

2.1 Experimental tests of CPT invariance

2.2 Experimental results for $\sin 2 \beta$

3.1 Central calorimeter parameters

3.2 End calorimeter parameters

4.1 SMT sensor parameters

7.1 Parameters used in proper lifetime fits

7.2 Results of likelihood fit to transverse proper decay length

7.3 Results of likelihood fit to three dimensional proper decay length

8.1 Measured effective tagging efficiency for CDF Run I, and the predicted efficiencies for CDF and DØ in Run II 


\section{Chapter 1}

\section{Introduction}

\subsection{The Standard Model and beyond}

The Standard Model of particle physics has proved to be an enormously successful theory and has weathered all experimental tests thrown at it for a long period of time. However, the theory is not without problems. It contains an unpleasantly large number of free parameters, and it fails to answer some of the questions about the fundamental nature of matter, such as:

1. Why are there three generations of fermions?

2. Why are the masses of the particles what they are?

In an attempt to improve on the Standard Model, various theories have been developed which extend it - supersymmetric theories are a particularly well known example of these ${ }^{1}$. To date there is still no convincing experimental evidence of any phenomena which requires any of these extensions.

\footnotetext{
${ }^{1}$ Although supersymmetric extensions to the standard model do not necessarily do anything to reduce the number of free parameters.
} 
In order to continue the process of testing the Standard Model and searching for physics beyond it, a number of new (or upgraded) accelerators and detectors have either recently begun operations or are now in the process of construction. These include

1. The asymmetric $e^{+} e^{-}$colliders PEP-II and KEKB and their associated detectors BABAR and Belle, which both began operating in 1999.

2. A luminosity upgrade to the $2 \mathrm{TeV}$ centre-of-mass energy proton-antiproton collider, the Tevatron, along with significant upgrades to its two general purpose detectors, DØ and CDF, which resumed operations early in 2001.

3. The $14 \mathrm{TeV}$ centre-of-mass energy proton-proton Large Hadron Collider (the LHC), with two general purpose detectors, ATLAS and CMS, and a dedicated b-physics experiment LHCb, which are scheduled to begin running in 2006.

These new detectors hope to explore fully the remaining parts of the Standard Model, and to discover evidence for the new theories which may replace or extend the Standard Model.

\subsection{The Tevatron upgrade}

The Tevatron accelerator originally began operations in 1983 providing a $1 \mathrm{TeV}$ proton beam to fixed target experiments. In 1985 it was first used as a protonantiproton collider running at up to $900 \mathrm{GeV}$ per beam. It has recently received a major upgrade to its capabilities, raising the centre of mass energy from 1.8 to 1.96 $\mathrm{TeV}$ and greatly increasing the luminosity to $\sim 2 \times 10^{32} \mathrm{~cm}^{-2} \mathrm{~s}^{-1}$ and beyond.

Table 1.1 gives the parameters of the Tevatron for Run I (1993-1995), Run IIa in both 396 ns and 132 ns bunch spacing modes, and for the projected high luminosity Run IIb. Run IIb operation is expected to begin in 2004 - the switch to $132 \mathrm{~ns}$ mode may wait until then, or it may begin earlier as part of Run IIa. 


\begin{tabular}{|l|c|c|c|c|l|}
\hline Run & Ib & $\begin{array}{c}\text { IIa } \\
(396 \mathrm{~ns})\end{array}$ & $\begin{array}{c}\text { IIa } \\
(132 \mathrm{~ns})\end{array}$ & IIb & \\
\hline \hline Proton bunches & 6 & 36 & 140 & 140 & \\
\hline $\begin{array}{l}\text { Protons / } \\
\text { bunch }\end{array}$ & $2.3 \times 10^{11}$ & $2.7 \times 10^{11}$ & $2.7 \times 10^{11}$ & $2.7 \times 10^{11}$ & \\
\hline $\begin{array}{l}\text { Antiproton } \\
\text { bunches }\end{array}$ & 6 & 36 & 103 & 103 & \\
\hline $\begin{array}{l}\text { Antiprotons / } \\
\text { bunch }\end{array}$ & $5.5 \times 10^{10}$ & $3.0 \times 10^{10}$ & $4.0 \times 10^{10}$ & $1.0 \times 10^{11}$ & \\
\hline $\begin{array}{l}\text { Total } \\
\text { antiprotons }\end{array}$ & $3.3 \times 10^{11}$ & $1.1 \times 10^{12}$ & $4.2 \times 10^{12}$ & $1.1 \times 10^{13}$ & \\
\hline $\begin{array}{l}\text { Typical } \\
\text { luminosity }\end{array}$ & $0.16 \times 10^{32}$ & $0.86 \times 10^{32}$ & $2.1 \times 10^{32}$ & $5.2 \times 10^{32}$ & $\mathrm{~cm}^{-2} \mathrm{~s}^{-1}$ \\
\hline $\begin{array}{l}\text { Integrated } \\
\text { luminosity }\end{array}$ & 3.2 & 17.3 & 42 & 105 & $\mathrm{pb}^{-1} / \mathrm{week}^{-1}$ \\
\hline Bunch spacing & 3500 & 396 & 132 & 132 & $\mathrm{nsec}$ \\
\hline $\begin{array}{l}\text { Interactions / } \\
\text { crossing }\end{array}$ & 2.5 & 2.3 & 1.9 & 4.8 & \\
\hline
\end{tabular}

Table 1.1: Tevatron parameters for Run I and Run II [1]

The upgrades to the accelerator include:

- Replacing the old Main Ring accelerator ${ }^{2}$ with a new $150 \mathrm{GeV}$ accelerator, the Main Injector, which is more suited to the task of supplying protons and anti-protons to the Tevatron ${ }^{3}$.

- A new antiproton storage ring, the Recycler, which shares the new tunnel with the Main Injector. The anti-proton intensity is the primary limitation on the luminosity at the Tevatron, and the Recycler is able to store a large number of anti-protons, and to recover the majority of the unspent anti-protons from the Tevatron after a store has completed.

\footnotetext{
${ }^{2}$ Which shared the tunnel with the Tevatron ring and actually passed through the calorimeter cryostat at $\mathrm{D} \varnothing$.
}

${ }^{3}$ The Main Injector is also able to deliver a $120 \mathrm{GeV}$ beam to fixed target experiments. 
- Other improvements to the anti-proton source to increase the anti-proton creation rate, and to the Tevatron to cope with the larger number of bunches and shorter time between bunch crossings in the machine.

\subsection{The $\mathrm{D} \emptyset$ upgrade}

The DØ experiment [2] originally began operation in 1992. The strengths of the original experiment were in its high precision calorimeter and the large angular coverage of the muon detectors. However the central tracking suffered from the absence of a solenoidal magnetic field in the central region. This prevented the measurement of the momentum of charged particles, and the determination of their charge.

To accompany the upgraded accelerator described in the previous section, the DØ experiment is also being upgraded $[3,4,5]$. The upgrade has been designed to enhance the tracking and triggering capabilities of the detector while building on the strengths of the experiment in calorimetry and muon coverage and to operate reliably at the higher luminosity and shorter bunch crossing times of the upgraded accelerator. Chapter 3 describes the upgraded detector.

The detector began Run II (first collisions were in April 2001) in a mechanically complete state, but with almost none of the fibre tracker and preshower detector readout electronics operational and a severely limited trigger and DAQ system. With only the silicon vertex detector, tracking abilities were severely limited. Commissioning work continued until October, when the readout electronics for the fibre tracker were installed during an accelerator shutdown. 


\subsection{The scope of this thesis}

This thesis describes the work I did helping to prepare for the start of Run II at the $\mathrm{D} \varnothing$ experiment. This work falls into two parts: provision of silicon and fibre tracker code for the reconstruction and Level 3 trigger software, and Monte Carlo simulation studies for $B$ meson reconstruction and lifetime measurement.

\subsubsection{Triggering and reconstruction software}

The data emerges from the detector as a long string of numbers. These are useless until they have been decoded, or 'unpacked' into some meaningful format. I wrote the majority of the unpacking code for the $\mathrm{D} \varnothing$ silicon and fibre trackers for the offline reconstruction program, and all of the central tracking unpacking code used in the Level 3 trigger. Additionally, I wrote the Level 3 trigger hit clustering algorithm for the fibre tracker and the first stage of the Level 3 clustering algorithm for the silicon tracker. The description of the raw data format for these detectors, the developement of the algorithms, and the results achieved with them are given in chapters 4 and 5 .

\subsubsection{B physics and CP violation}

One of the subjects the $\mathrm{D} \varnothing$ detector will be used for is the study of $B$ mesons, in particular to measure $\mathrm{CP}$ violation in the $B^{0} \bar{B}^{0}$ system. As a contribution towards this, I conducted a Monte Carlo study into the reconstruction of $B^{+}$mesons, and the measurement of their lifetime. This involved developing mass constrained track fitting code within the D $\varnothing$ software environment, and writing a maximum likelihood fitting routine in order to extract the lifetimes. Chapter 2 contains some of the theory involved in CP violation, and chapters 6 and 7 give the results of the Monte Carlo study. 


\section{Chapter 2}

\section{$\mathrm{CP}$ violation, $B$ mesons, and the CKM model}

\subsection{Introduction to $\mathrm{CP}$ violation}

The concept of symmetries is the basis for much of modern quantum theory. The three discrete symmetries $\mathrm{C}$ (particle-antiparticle conjugation), $\mathrm{P}$ (parity) and $\mathrm{T}$ (time reversal) have all played a significant role since the beginning of quantum mechanics. The CPT theorem of quantum field theory states that all physics is invariant under the combined transformation CPT. However the separate symmetries are not necessarily conserved. Parity violation was first observed in 1957 [6], and it was soon established that the weak interaction involves eigenstates of $\mathrm{CP}$ rather than C and P separately. The discovery, in 1964 [7], that CP was violated in the decays of neutral kaons was therefore surprising, and even today CP violation is not well understood. After its discovery in the kaon system, it is only recently that it has been observed $[8,9,10]$ within the neutral $B$ system, and although it can be accommodated within the Standard Model it remains among the least well tested parts of the model. 
$\mathrm{CP}$ violation has an additional importance in cosmology, as it is one of the proposed mechanisms which seek to explain the dominance of matter over antimatter in the Universe.

\subsubsection{CP violation in neutral kaons}

Experimentally, two neutral kaon states, $K_{S}$ and $K_{L}$ with short and long lifetimes respectively, have been observed. These were originally believed to be the $\mathrm{CP}$ eigenstates $\left|K_{1}^{0}\right\rangle(\mathrm{CP}=+1)$ and $\left|K_{2}^{0}\right\rangle(\mathrm{CP}=-1) . K_{S}$ should therefore decay only to $\mathrm{CP}$ +1 states, such as 2 pions, and $K_{L}$ only to $\mathrm{CP}-1$ states, such as 3 pions ${ }^{1}$. However, it was found [7] that $K_{L}$ sometimes decays as $K_{L} \rightarrow 2 \pi$. Later on, CP violation was also observed in the semi-leptonic decay $K_{L} \rightarrow \pi^{\mp} \ell^{ \pm} \nu$. Therefore $K_{L}$ and $K_{S}$ are not the pure CP eigenstates, and the weak interaction does not necessarily conserve CP symmetry.

The experimental evidence for $\mathrm{CP}$ violation with neutral kaons is in the non-zero values of the following decay amplitudes

$$
\begin{aligned}
\eta_{+-} & =\frac{A\left(K_{L} \rightarrow \pi^{+} \pi^{-}\right)}{A\left(K_{S} \rightarrow \pi^{+} \pi^{-}\right)} \\
& =\left|\eta_{+-}\right| e^{i \phi_{+-}} \\
\eta_{00} & =\frac{A\left(K_{L} \rightarrow \pi^{0} \pi^{0}\right)}{A\left(K_{S} \rightarrow \pi^{0} \pi^{0}\right)} \\
& =\left|\eta_{00}\right| e^{i \phi_{00}} \\
\delta(\ell) & =\frac{\Gamma\left(K_{L} \rightarrow \pi^{-} \ell^{+} \nu\right)-\Gamma\left(K_{L} \rightarrow \pi^{+} \ell^{-} \nu\right)}{\Gamma\left(K_{L} \rightarrow \pi^{-} \ell^{+} \nu\right)+\Gamma\left(K_{L} \rightarrow \pi^{+} \ell^{-} \nu\right)}
\end{aligned}
$$

\footnotetext{
${ }^{1}$ Actually, the three pion state $\pi^{+} \pi^{-} \pi^{0}$ can be a $\mathrm{CP}=+1$ eigenstate (if the orbital angular momentum of the pions is $>0$ then both $\mathrm{CP}=+1$ and -1 are possible), and $K_{S}$ does decay to this with a small branching ratio. However, there are no 2 pion states with $\mathrm{CP}=-1$ and a $K_{L}$ decaying to 2 pions is an absolute sign of CP violation. Additionally, the decay $K_{S} \rightarrow \pi^{0} \pi^{0} \pi^{0}$ must be $\mathrm{CP}$ violating, but has so far not been experimentally demonstrated [11].
} 
The current world average values for these are [12]

$$
\begin{aligned}
\left|\eta_{+-}\right| & =(2.276 \pm 0.017) \times 10^{-3} & \phi_{+-} & =(43.3 \pm 0.5)^{\circ} \\
\left|\eta_{00}\right| & =(2.262 \pm 0.017) \times 10^{-3} & \phi_{00} & =(43.2 \pm 1.0)^{\circ} \\
\delta(e) & =(0.304 \pm 0.025) \% & \delta(\mu) & =(0.333 \pm 0.014) \%
\end{aligned}
$$

The neutral kaon system thus provides clear evidence that CP is not conserved in nature.

\section{$2.2 \quad$ Discrete symmetries}

Parity, charge conjugation and time reversal are all discrete symmetries. Such symmetries can be represented by operators in quantum mechanics [13].

\subsubsection{C and P symmetry}

$\mathrm{C}$ and $\mathrm{P}$ may be represented by operators $\mathcal{C}$ and $\mathcal{P}$ which have similar properties. For $\mathrm{C}$ and $\mathrm{P}$ to be good quantum numbers, the operators $\mathcal{C}$ and $\mathcal{P}$ must commute with the Hamiltonian, so

$$
[\mathcal{P}, H]=0, \quad[\mathcal{C}, H]=0
$$

Since the weak interaction violates parity, equation (2.1) cannot hold, and so no operators $\mathcal{P}$ or $\mathcal{C}$ can be defined for it. However, the electromagnetic interactions do conserve parity. This means that $\mathcal{P}$ can be defined by only considering the kinematic and electromagnetic part of the overall Lagrangian. Other pieces of the Lagrangian can then be tested to see if they are invariant under $\mathcal{P}$ - if not then they are parity violating. Similarly $\mathcal{C}$ can be defined for the kinematic and electromagnetic 
Lagrangian only, and terms in the Lagrangian which are not invariant under $\mathcal{C}$ violate C.

\section{Properties of $\mathbf{P}$}

$\mathcal{P}$ is unitary, so that

$$
\mathcal{P}^{\dagger}=\mathcal{P}^{-1}
$$

Acting on a state twice with $\mathcal{P}$ returns it to the original state

$$
\mathcal{P}^{2}|\mathbf{r}\rangle=|\mathbf{r}\rangle
$$

Parity is to a large extent arbitary. For example, the proton, neutron and $\Lambda$ are all assigned parity +1 and this determines the parity of all other mesons and baryons containing $u, d$ and $s$ quarks. However, for the case of mesons which contain a quark and anti-quark of the same flavour, the parity is absolute (the pion has parity -1).

\section{Properties of $\mathrm{C}$}

$\mathcal{C}$ is also unitary, and charge conjugating a state twice gives the original state

$$
\mathcal{C}^{2}\left|e^{-}\right\rangle=\left|e^{-}\right\rangle
$$

Therefore

$$
\begin{aligned}
& \mathcal{C}\left|e^{-}\right\rangle=e^{i \theta}\left|e^{+}\right\rangle \\
& \mathcal{C}\left|e^{+}\right\rangle=e^{-i \theta}\left|e^{-}\right\rangle
\end{aligned}
$$


$\mathcal{C}$ does not commute with the charge operator $\mathcal{Q}$ as

$$
\begin{aligned}
& \mathcal{Q}\left|e^{-}\right\rangle=-e\left|e^{-}\right\rangle \\
& \mathcal{Q}\left|e^{+}\right\rangle=+e\left|e^{+}\right\rangle
\end{aligned}
$$

Particles in a simultaneous eigenstate of $\mathcal{H}$ and $\mathcal{Q}$ cannot be in a simultaneous eigenstate of $\mathcal{C}$, so only those with zero electric charge can have a definite value of C. This is true not only for electric charge, but also for any other sort of conserved charge, such as lepton number, baryon number, strangeness and any other quantum number conserved by the electromagnetic and strong forces. Thus, the $\pi^{0}$ and photon are eigenstates of $\mathcal{C}$, but the neutron and neutrino are not.

\subsubsection{T symmetry}

The time reversal operator is more complicated than parity or charge conjugation. Under the translation $t \rightarrow-t$ the Schrödinger equation transforms to

$$
i \frac{\partial \psi}{\partial t}=-\frac{\nabla^{2} \psi}{2 m} \quad \rightarrow \quad-i \frac{\partial \psi}{\partial t}=-\frac{\nabla^{2} \psi}{2 m}
$$

In classical mechanics the motion of a free particle is invariant under time reversal, so this property should hold in quantum mechanics too. The invariance can be retained if the time reversal is a combination of $t \rightarrow-t$ and complex conjugation, which gives the time reversed equation

$$
i \frac{\partial \psi^{*}}{\partial t}=-\frac{\nabla^{2} \psi^{*}}{2 m}
$$

$\mathcal{T}$ is therefore not a unitary operator, but an antiunitary operator. An antiunitary operator can be represented as the product of a unitary operator and an operator which gives the complex conjugate of everything to the right of it. 
$\mathcal{T}$ does not have meaningful eigenvalues, for example if

$$
\mathcal{T}|\psi\rangle=k|\psi\rangle
$$

where $k$ is a complex number, then

$$
\mathcal{T}\left(e^{i \zeta}|\psi\rangle\right)=e^{-i \zeta} \mathcal{T}|\psi\rangle=k e^{-2 i \zeta}\left(e^{i \zeta}|\psi\rangle\right)
$$

but as $|\psi\rangle$ and $e^{i \zeta}|\psi\rangle$ represent the same state, they cannot have different eigenvalues.

Because of this, $\mathrm{T}$ invariance does not lead to a conservation law. There is no quantum number to be conserved in a $\mathrm{T}$ invariant system.

\subsection{The CPT theorem}

The CPT theorem states that any quantum field theory which obeys certain general properties, including Lorentz invariance and local (anti-)commutation relations obeying the spin-statistics connection, is CPT invariant. It is very difficult to produce a realistic quantum field theory in which CPT is violated, and so it is generally assumed that the CPT invariance holds. However it is possible that future theories might not possess CPT invariance. String theory is non-local and may also display spontaneous breaking of the Lorentz symmetry, and so might violate CPT.

If CPT invariance does hold then it has the consequence that violating one of the individual symmetries implies the violation of the others. If $\mathrm{CP}$ is violated then $\mathrm{T}$ must be violated also. However, the CPT theorem applies to quantum field theories, not to observables. For example, an observation of CP violation does not imply that $\mathrm{T}$ is also violated in that process, but it does imply that the Lagrangian for the process must violate both $\mathrm{CP}$ and $\mathrm{T}$.

The CPT theorem predicts certain experimentally testable predictions. The most basic of these is that the mass and lifetime of a particle must be equal to those 
of its antiparticle. Another consequence is that the magnetic dipole moment for the particle should be opposite to that of the antiparticle, which is usually given in terms of the gyromagnetic moment $g$. These properties have been measured for some particles, as shown in table 2.1; there is no significant violation of CPT invariance.

\subsection{CP violation in the Standard Model}

If CPT symmetry is conserved, but CP is violated, then time reversal must also be violated. Due to the antiunitary nature of the time reversal operator the Hamiltonian of a $\mathrm{CP}$ violating interaction must be complex - the time reversed form is then different from the original form.

This can be introduced in the Standard Model by considering the Lagrangian for the weak interaction and the Yukawa couplings of the quarks $[13,14,15]$. The left handed quarks form an $S U(2)$ doublet

$$
q_{L}^{\prime}=\left(\begin{array}{c}
u_{L}^{\prime} \\
d_{L}^{\prime}
\end{array}\right)
$$

where the primes indicate that these are mixtures of the mass eigenstates (see later). The right handed quarks are $S U(2)$ singlets, $u_{R}^{\prime}$ and $d_{R}^{\prime}$. The Lagrangian for the

\begin{tabular}{|c|c|c|c|}
\hline$\psi$ & $\left(m_{\psi}-m_{\bar{\psi}}\right) / m_{\text {average }}$ & $\left(\tau_{\psi}-\tau_{\bar{\psi}}\right) / \tau_{\text {average }}$ & $\left(g_{\psi}-g_{\bar{\psi}}\right) / g_{\text {average }}$ \\
\hline$e^{+}$ & $<8 \times 10^{-9}$ & - & $(-0.5 \pm 2.1) \times 10^{-12}$ \\
$\mu^{+}$ & - & $(2 \pm 8) \times 10^{-5}$ & $(-2.6 \pm 1.6) \times 10^{-8}$ \\
$\pi^{+}$ & $(2 \pm 5) \times 10^{-4}$ & $(6 \pm 7) \times 10^{-4}$ & - \\
$n$ & $(9 \pm 5) \times 10^{-5}$ & - & - \\
\hline
\end{tabular}

Table 2.1: Experimental tests of CPT invariance [12] 
charged current weak interaction is

$$
\mathcal{L}_{\text {c. с. }}=\frac{g}{\sqrt{2}}\left(W_{\mu}^{+} \bar{u}_{L}^{\prime} \gamma^{\mu} d_{L}^{\prime}+W_{\mu}^{-} \bar{d}_{L}^{\prime} \gamma^{\mu} u_{L}^{\prime}\right)
$$

and for the neutral current weak interaction is

$$
\begin{aligned}
\mathcal{L}_{\text {n. c. }}= & \frac{2}{2 \cos \theta_{W}} Z_{\mu}\left(\bar{u}_{L}^{\prime} \gamma^{\mu} u_{L}^{\prime}-\bar{d}_{L}^{\prime} \gamma^{\mu} d_{L}^{\prime}\right. \\
& \left.-\frac{\sin ^{2} \theta_{W}}{3}\left(2\left(\bar{u}_{L}^{\prime} \gamma^{\mu} u_{L}^{\prime}+\bar{d}_{L}^{\prime} \gamma^{\mu} d_{L}^{\prime}\right)-\left(\bar{u}_{R}^{\prime} \gamma^{\mu} u_{R}^{\prime}+\bar{d}_{R}^{\prime} \gamma^{\mu} d_{R}^{\prime}\right)\right)\right)
\end{aligned}
$$

The masses of the quarks are due to the Yukawa couplings with the vacuum expectation value of the Higgs field

$$
\mathcal{L}_{\mathrm{Y}}=-\bar{d}_{L}^{\prime} M_{d}^{\prime} d_{R}^{\prime}-\bar{u}_{L}^{\prime} M_{u}^{\prime} u_{R}^{\prime}+\text { h. c. }
$$

where 'h. c.' denotes the hermitian conjugate.

There are at least three generations of quarks, and experimentally there is good evidence that there are only three $[16,17,18]$, so the quark states $q^{\prime}$ are actually $3 \times 1$ column matrices and the mass terms $M^{\prime}$ are $3 \times 3$ matrices in generation space. The Standard Model provides no constraints on the flavour structure of the Yukawa couplings and so $M_{d}^{\prime}$ and $M_{u}^{\prime}$ are completely arbitrary. They can, however, be diagonalised by a bi-unitary transformation

$$
\begin{aligned}
& M_{u}=U_{L}^{u^{\prime \dagger}} M_{u}^{\prime} U_{L}^{u^{\prime}}=\operatorname{diag}\left(m_{u}, m_{c}, m_{t}\right) \\
& M_{d}=U_{L}^{d^{\prime} \dagger} M_{d}^{\prime} U_{L}^{d^{\prime}}=\operatorname{diag}\left(m_{d}, m_{s}, m_{b}\right)
\end{aligned}
$$

The quark mass eigenstates are therefore not the same as the eigenstates of the weak interaction, and the charged current (2.14) can be rewritten

$$
\mathcal{L}_{\text {c. c. }}=\frac{g}{\sqrt{2}}\left(W_{\mu}^{+} \bar{u}_{L} \gamma^{\mu} V d_{L}+W_{\mu}^{-} \bar{d}_{L} \gamma^{\mu} V^{\dagger} u_{L}\right)
$$


where $V$ is the Cabibbo-Kobayashi-Maskawa (CKM) matrix [19]

$$
V=U_{L}^{u^{\prime \dagger}} U_{L}^{d^{\prime}}=\left(\begin{array}{ccc}
V_{\mathrm{ud}} & V_{\mathrm{us}} & V_{\mathrm{ub}} \\
V_{\mathrm{cd}} & V_{\mathrm{cs}} & V_{\mathrm{cb}} \\
V_{\mathrm{td}} & V_{\mathrm{ts}} & V_{\mathrm{tb}}
\end{array}\right)
$$

Here $V_{i j}$ is the matrix element coupling the $i$ th up type quark to the $j$ th down type quark.

Substituting the mass eigenstates into the neutral current (2.15) produces the trivial mixing matrix $V_{\text {n. c. }}=U_{L}^{u^{\prime} \dagger} U_{L}^{u^{\prime}}=U_{L}^{d^{\prime} \dagger} U_{L}^{d^{\prime}}=1$, so there are no flavour changing neutral currents (at tree level).

Although the CKM matrix has 18 parameters the constraints of unitarity and the fact that overall phases are irrelevant means that there are only 4 independent parameters. This is usually shown explicitly by using a specific parameterization of the matrix. The approximate Wolfenstein parameterization [20] is common

$$
\begin{aligned}
V_{\mathrm{CKM}} & =\left(\begin{array}{ccc}
1-\frac{1}{2} \lambda^{2} & \lambda & A(\rho-i \eta) \lambda^{3} \\
-\lambda & 1-\frac{1}{2} \lambda^{2} & A \lambda^{2} \\
A(1-\rho-i \eta) \lambda^{3} & -A \lambda^{2} & 1
\end{array}\right) \\
& +\left(\begin{array}{ccc}
-\frac{1}{8} \lambda^{4} & 0 & 0 \\
A^{2}\left(\frac{1}{2}-\rho-i \eta\right) \lambda^{5} & -\frac{1}{8}\left(1+4 A^{2}\right) \lambda^{4} & 0 \\
\frac{1}{2} A(\rho+i \eta) \lambda^{5} & A\left(\frac{1}{2}-\rho-i \eta\right) \lambda^{4} & -\frac{1}{2} A^{2} \lambda^{4}
\end{array}\right)+\mathcal{O}\left(\lambda^{6}\right)
\end{aligned}
$$

with $\lambda=\sin \theta_{\mathrm{C}}$, the Cabbibo angle.

The unitarity constraints can also be shown in graphical form [21]. There are six constraints of the form

$$
V_{\mathrm{ud}} V_{\mathrm{ub}}^{*}+V_{\mathrm{cd}} V_{\mathrm{cb}}^{*}+V_{\mathrm{td}} V_{\mathrm{tb}}^{*}=0
$$

This particular condition is interesting because $V_{\mathrm{ud}} \simeq V_{\mathrm{tb}} \simeq 1$. When drawn in the complex plane and rescaled by dividing through by $V_{\mathrm{cd}} V_{\mathrm{cb}}^{*}$, the condition becomes 
a triangle in the complex plane with vertices at $(0,0),(1,0)$ and $(\bar{\rho}, \bar{\eta})$, with $\bar{\rho}=$ $\rho\left(1-\frac{1}{2} \lambda^{2}\right), \bar{\eta}=\eta\left(1-\frac{1}{2} \lambda^{2}\right.$ ) (see figure 2.1). Although there are six possible unitarity triangles, this one is commonly referred to as the unitarity triangle.

The unitarity triangle can be used as a test of how well the Standard Model describes $\mathrm{CP}$ violation. If the three sides do not join up to form a triangle, then the requirement of unitarity has been broken. This would indicate the existence of physics beyond the Standard Model [14, 22].

\subsection{CP violating processes}

There are three basic types of $\mathrm{CP}$ violation that can occur in the decay of mesons $[13,15]$. These are:

1. $\mathrm{CP}$ violation in the mixing of neutral mesons.

2. CP violation in the decay, which occurs for both neutral and charged mesons.

3. CP violation in the interference of decays with and without mixing.

\subsubsection{CP violation in mixing}

$\mathrm{CP}$ violation can occur in the mixing of neutral mesons, such as kaons. In this case the mass eigenstates are not CP eigenstates. The mixing is due to box diagrams such

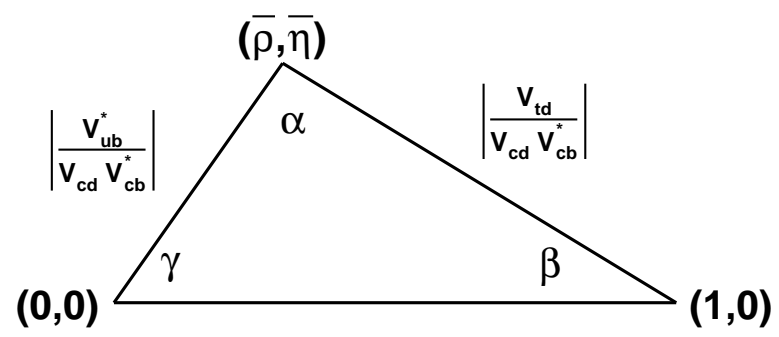

Figure 2.1: The CKM unitarity triangle in the Wolfenstein parameterization 
as in figure 2.2. This is the predominant cause of $\mathrm{CP}$ violation in the neutral kaon system. It appears as a difference in the mixing rates for $K^{0} \rightarrow \bar{K}^{0}$ and $\bar{K}^{0} \rightarrow K^{0}$. Since the mixing is through $c$ quarks for kaons the CP violation depends on the magnitude of the complex component of the $V_{c d}$ CKM element, which is small, but in the neutral $B$ system the mixing is mediated by $t$ quarks and the $V_{t d}$ element creates a much larger $\mathrm{CP}$ violating effect. This type of $\mathrm{CP}$ violation is called $C P$ violation in the mixing, or indirect CP violation.

\subsubsection{CP violation in the decay}

Because CP violation enters the Standard Model as a phase it only appears as an interference effect. One way to get such interference is between the partial decay rates $\mathrm{X} \rightarrow f$ and $\overline{\mathrm{X}} \rightarrow \bar{f}$. The decay amplitudes are

$$
A=\left\langle f\left|\mathcal{H}_{\text {weak }}\right| \mathrm{X}\right\rangle \quad \bar{A}=\left\langle\bar{f}\left|\mathcal{H}_{\text {weak }}\right| \overline{\mathrm{X}}\right\rangle
$$

If two processes contribute to this decay then

$$
\begin{aligned}
& A_{\text {total }}=\left|A_{1}\right| e^{i \Delta_{1}} e^{i \Phi_{1}}+\left|A_{2}\right| e^{i \Delta_{2}} e^{i \Phi_{2}} \\
& \bar{A}_{\text {total }}=\left|A_{1}\right| e^{i \Delta_{1}} e^{-i \Phi_{1}}+\left|A_{2}\right| e^{i \Delta_{2}} e^{-i \Phi_{2}}
\end{aligned}
$$

where $\Delta_{i}$ is the 'strong phase' due to final state interactions, and $\Phi_{i}$ is the 'weak phase' from the CKM matrix, and so opposite in sign between the particle and
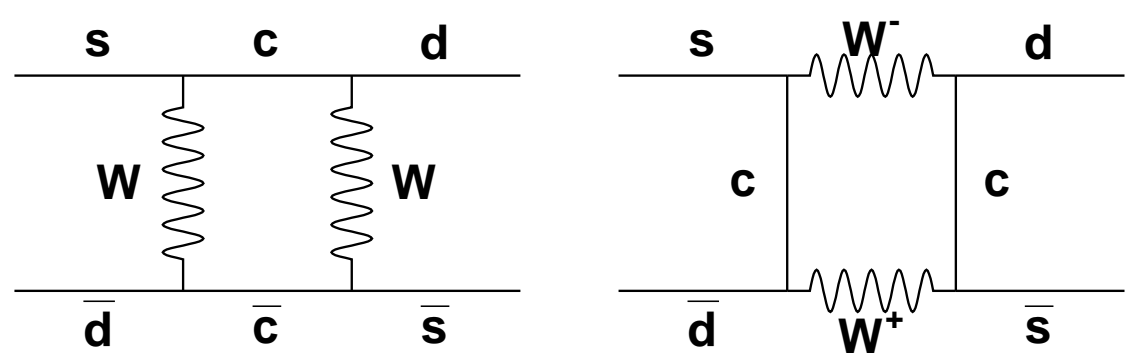

Figure 2.2: Box diagrams for $\bar{K}^{0} \rightarrow K^{0}$ mixing via top quarks 
anti-particle decays. Therefore

$$
\begin{aligned}
& \left|A_{\text {total }}\right|^{2}=\left|A_{1}\right|^{2}+\left|A_{2}\right|^{2}+\left|A_{1}\right|\left|A_{2}\right| \cos (\delta+\phi) \\
& \left|\bar{A}_{\text {total }}\right|^{2}=\left|A_{1}\right|^{2}+\left|A_{2}\right|^{2}+\left|A_{1}\right|\left|A_{2}\right| \cos (\delta-\phi)
\end{aligned}
$$

with $\delta=\Delta_{2}-\Delta_{1}$ and $\phi=\Phi_{2}-\Phi_{1}$. Hence

$$
\left|\bar{A}_{\text {total }}\right|^{2}-\left|A_{\text {total }}\right|^{2}=4\left|A_{1}\right|\left|A_{2}\right| \sin \delta \sin \phi
$$

There is an asymmetry in the decay rates if both $\phi \neq 0$ and $\delta \neq 0$. This form of $\mathrm{CP}$ violation is called $C P$ violation in the decay or direct $C P$ violation. Unlike $\mathrm{CP}$ violation in the mixing, this can occur with charged mesons as well as neutral ones.

\subsubsection{CP violation in the interference of mixing and decay}

If a neutral meson and its anti-particle can both decay to the same final state $f$ then interference can occur between the mixing and decay amplitudes, for example between

$$
\mathrm{X} \rightarrow f \quad \text { and } \quad \mathrm{X} \rightarrow \overline{\mathrm{X}} \rightarrow f
$$

This type of CP violation is called CP violation in the interference of mixing and decay.

The theoretically clean situation is when $f$ is a $\mathrm{CP}$ eigenstate and $\mathrm{CP}$ is conserved in the mixing and the decay separately (for example $K_{L} \rightarrow \pi^{0} \nu \bar{\nu}$ and $B^{0} \rightarrow J / \psi K_{S}$ ). The CP effects are then purely due to the phase in the CKM matrix. 


\section{6 $\quad B$ meson decays}

\subsubsection{Production at the Tevatron}

The leading order QCD processes in heavy quark production are shown in figure 2.3 [23]. The dominant process for the production of $b$ quarks at the Tevatron centre of mass energy is gluon-gluon fusion (as opposed to top quark production, where quark-antiquark annihilation dominates) [24].

Among the predictions of leading order QCD is that the heavy quark and antiquark are produced back to back in the parton-parton rest frame, and so back to back in the plane transverse to the collider beam. The average transverse momentum of the quark is approximately equal to its mass $\left\langle p_{T}(q)\right\rangle \sim m_{q}$, so the average transverse $b$ quark momentum is $\sim 4-5 \mathrm{GeV}$.

However, the next to leading order 'corrections' to the calculations can be as large, or larger, than the leading order terms [23]. This leads to significant departures from the behaviour described by leading order calculations, including $b \bar{b}$ pairs produced close in phase space rather than back to back in the parton-parton frame. Even the next to leading order computation is not totally satisfactory, as the experimental results [25] for the cross-section agree in shape, but are greater in value, than the calculated quantity [26].

\subsubsection{Lifetimes}

Measurements of the lifetimes of $B$ mesons are needed to determine the CKM matrix element $\left|V_{c b}\right|$ or to obtain the time dependence of $B \bar{B}$ oscillations. The simplest model of $B$ decays is the spectator quark model, where the $b$ quark decays to a $c$ quark by emitting a $W$ boson, which converts to either a $\ell \nu$ or $q \bar{q}$ pair, while the 'other' quark in the $B$ meson plays no part in the interaction. In this model, the $b$ quark decays as a free particle (see figure 2.4), and the lifetime of any $B$ meson 

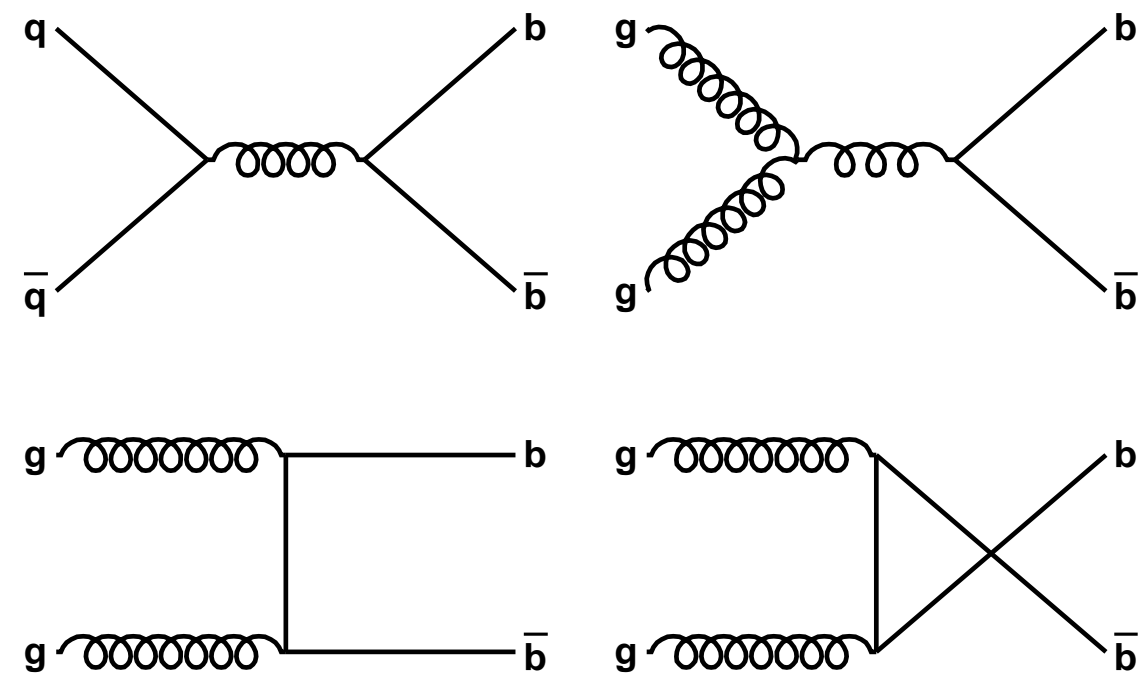

Figure 2.3: Leading order diagrams for $b \bar{b}$ production

would be given as (in analogy to the muon lifetime)

$$
\Gamma=\frac{G_{F}^{2} m_{b}^{2}}{192 \pi^{3}} \cdot\left|V_{c b}\right|^{2} \cdot \mathcal{F}
$$

where $\mathcal{F}$ is a phase space factor. (This neglects $b \rightarrow u$ transitions, equivalent to assuming $\left|V_{u b}\right|^{2}$ is small.)

The spectator model also predicts the lifetime of charm hadrons to be equal, but those that have been measured [12] show significant differences, for example

$$
\tau\left(D^{-}\right) \sim 2.5 \tau\left(D^{0}\right)
$$

This implies that there are very significant non-spectator effects present in the decay of charmed mesons, and it therefore seems likely that there are also non-spectator effects present in $B$ decays, and thus lifetime differences between the different $B$ mesons. Figure 2.5 shows examples of non-spectator decays which will give rise to differing lifetimes for different $B$ mesons. However, the $b$ quark mass is significantly greater than the $c$ quark mass, and this is expected to suppress the non-spectator 


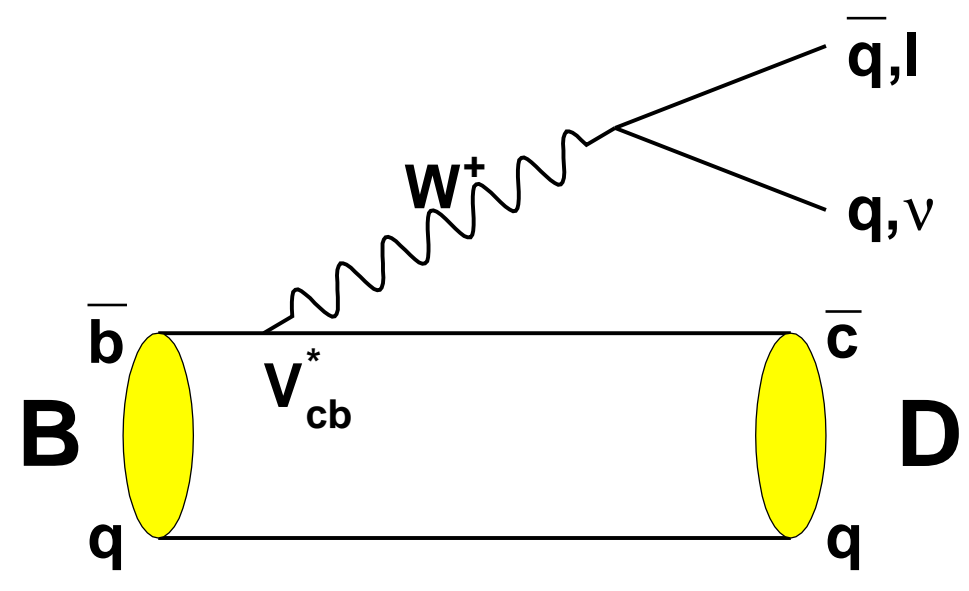

Figure 2.4: Spectator model of $B$ decay

effects, making the $B$ lifetime differences much less than those for charm mesons.

The heavy quark expansion technique has been used to predict the lifetime differences between the different $B$ mesons $[27,28]$. The expected difference between $B^{0}$ and $B^{+}$is of the order of $5-10 \%$, and between $B^{0}$ and $B_{s}^{0}$ a much smaller difference of less than $1 \%$.

The current world average measurements of the $B$ meson lifetimes are [12]

$$
\begin{aligned}
\tau\left(B^{+}\right) & =(1.655 \pm 0.027) \mathrm{ps} \\
\tau\left(B^{0}\right) & =(1.540 \pm 0.024) \mathrm{ps} \\
\tau\left(B^{+}\right) / \tau\left(B^{0}\right) & =1.074 \pm 0.028 \\
\tau\left(B_{s}^{0}\right) & =(1.464 \pm 0.057) \mathrm{ps}
\end{aligned}
$$

\subsubsection{CP violation in the $B$ meson system}

The neutral $B$ meson system is expected to provide a much better determination of the CKM matrix parameters relating to $\mathrm{CP}$ violation than the neutral kaon system. This is because the $\mathrm{CP}$ violating effects are much larger for neutral $B$ s than for 


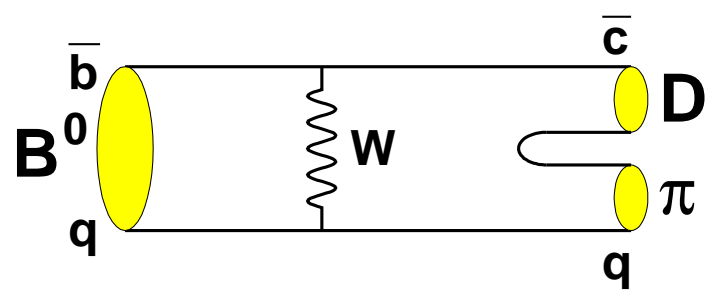

(a) $B^{0}$ decaying through internal $W$ exchange

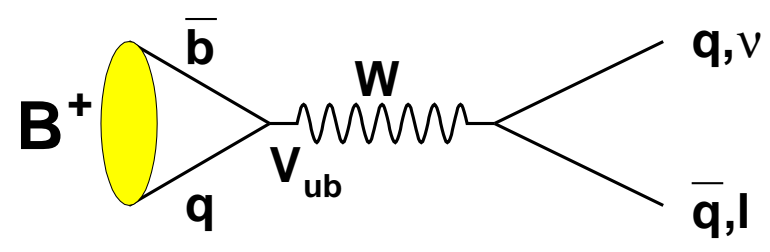

(b) $B^{+}$annihilation

Figure 2.5: Non-spectator $B$ decays leading to different lifetimes for different $B$ mesons

neutral $K \mathrm{~s}[15,29]$.

The $B$ meson system differs in many respects from the kaon system. There are two types of neutral $B$ meson $B_{d}^{0}(\equiv \bar{b} d)$ and $B_{s}^{0}(\equiv \bar{b} s)$. Whereas with kaons the large difference in phase space between the final states of $K_{S}$ and $K_{L}$ gives rise to a large lifetime difference, with the $B$ system the vast majority of final states are the same for both $B^{0}$ and $\bar{B}^{0}$, and so the lifetime difference is small. The mass difference is much larger than for the kaons, with the difference in the $B_{d}^{0}$ system being $\Delta M_{d}=(0.472 \pm 0.017) \times 10^{12} \hbar \mathrm{s}^{-1}$, and the difference in the $B_{s}^{0}$ system $\Delta M_{s}=>10.6 \times 10^{12} \hbar \mathrm{s}^{-1}$. The oscillation parameters are $x_{d}=\Delta M_{d} / \Gamma_{d}=$ $0.730 \pm 0.029$ for the $B_{d}^{0}$ and $x_{s}=\Delta M_{s} / \Gamma_{s}>15.7$ for the $B_{s}^{0}[12]$.

In the $B$ system the amount of indirect $\mathrm{CP}$ violation is predicted to be small $\left(<10^{-3}\right)$, and there has so far been no measured evidence of direct CP violation $[30,31]$. The most theoretically clean processes are in the interference of mixing and decay to a $\mathrm{CP}$ eigenstate. In this mode, the time dependent rates 
$\Gamma(t)=\Gamma\left(B^{0}(t) \rightarrow f\right)$ and $\bar{\Gamma}(t)=\Gamma\left(\bar{B}^{0}(t) \rightarrow f\right)$ are given by

$$
\begin{aligned}
& \Gamma(t) \sim e^{-\Gamma t}\left(1+R \cos \left(\Delta M_{d}\right)-I \sin \left(\Delta M_{d} t\right)\right) \\
& \bar{\Gamma}(t) \sim e^{-\Gamma t}\left(1-R \cos \left(\Delta M_{d}\right)+I \sin \left(\Delta M_{d} t\right)\right)
\end{aligned}
$$

where $R$ is the amount of direct $\mathrm{CP}$ violation and $I$ is the amount of interference $\mathrm{CP}$ violation.

For example, in the decay $B^{0} \rightarrow J / \psi K_{S}$ the only contribution to the weak phase is due to a single tree level diagram, and this has $R=0$ and $I=\sin 2 \beta$. However, for $B^{0} \rightarrow \pi^{+} \pi^{-}$there is an additional contribution from the 'penguin' diagram shown in figure 2.6. This penguin diagram has different strong and weak phases from the tree diagram, and so here $R \neq 0$. For this decay, $I=\sin 2(\alpha+\delta)$ where $\delta$ is a phase shift due to the direct $\mathrm{CP}$ violation. Unfortunately the theoretical calculation of $\delta$ is difficult $[29,32]$.
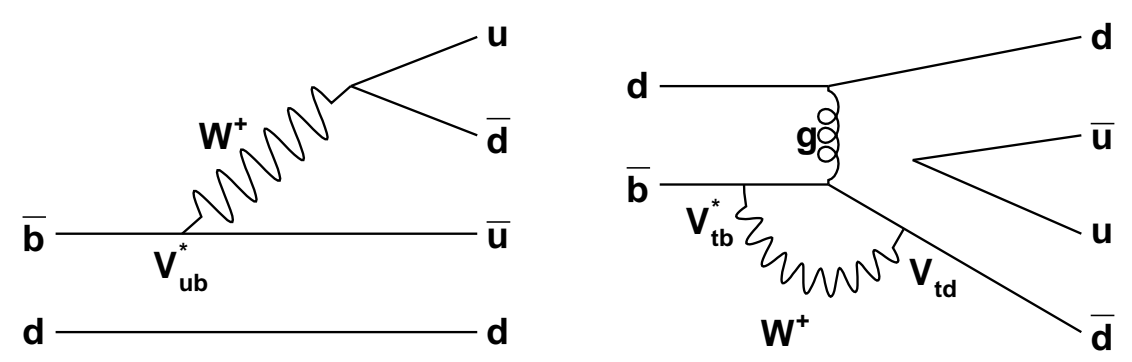

Figure 2.6: Tree and penguin diagrams for $B^{0} \rightarrow \pi^{+} \pi^{-}$

\subsection{Experimental results}

The Standard Model explains CP violation in terms of the phase in the CKM matrix. Testing the model involves measuring the magnitude of the CKM matrix elements and determining the relative phases. There are a number of existing and planned experiments which aim to measure the CKM parameters accurately enough to over- 
constrain the model and determine whether it does fully describe the phenomenon of $\mathrm{CP}$ violation, or whether a new model is required.

\subsubsection{Past and current and future experiments}

Up until very recently the study of CP violation was mainly carried out at fixed target experiments studying $K$ mesons, and the latest generation of these experiments, $\mathrm{KTeV}$ at Fermilab and NA48 at CERN, are still producing results. However, due to the small magnitude of the CP violating effects using kaons, recently attention has turned to studying $\mathrm{CP}$ violation using $B$ mesons. Because of the short lifetime of the $B \mathrm{~s}$, studying their decays in detail requires the use of precision vertex detectors, a technique pioneered at the TASSO detector at DESY [33].

The majority of $B$ physics experiments so far have been based at $e^{+} e^{-}$colliders, operating either at the $\Upsilon(4 S)$ resonance (which has a branching ratio of $\sim 96 \%$ to $B^{+}$and $B^{0}$ ), such as the CLEO detector at Cornell, or at the $Z^{0}$ pole, like the LEP experiments. Additionally, some $B$ physics studies were done at hadron colliders, particularly by the CDF experiment at Fermilab.

None of these experiments were able to measure $\mathrm{CP}$ violation in the $B$ meson system (although CDF was able to produce a result for $\sin 2 \beta$, albeit with a very large error [8]). Measuring $\mathrm{CP}$ violation at a symmetric collider at the $\Upsilon(4 S)$ is not possible because the $B \bar{B}$ pairs produced are in a coherent state and almost stationary. The coherent state means that it is necessary to measure the difference in the decay lengths of the two $B \mathrm{~s}$, and the fact that they are almost stationary makes this length too small to measure accurately. Because of this, the new ' $B$ factories', PEPII at SLAC and KEKB at KEK, are both asymmetric $e^{+} e^{-}$colliders at the $\Upsilon(4 S)$ resonance. The asymmetric beam energies mean that the $B$ s are produced with a Lorentz boost in the frame of the detector, and consequently the difference in their decay lengths is large enough to be measured. The detectors for each collider, BABAR and Belle, both began taking data in 1999 and have published their first 
results (see the next section).

In addition to the $B$ factories, Run II of the Tevatron will provide an opportunity to measure $\mathrm{CP}$ violation from $p \bar{p}$ collisions. Both the $\mathrm{D} \varnothing$ and CDF detectors have been upgraded, and both hope to be able to measure $\sin 2 \beta$ to comparable accuracy to the $B$ factories. Additionally, the $\Upsilon(4 s)$ does not decay to $B_{s}$ mesons at all, so the Tevatron experiments are the only current experiments that can study these.

In the longer term, there are two dedicated $B$ experiments planned for hadron colliders - LHCb planned for the $14 \mathrm{TeV}$ pp LHC collider at CERN, and BTeV as a future Tevatron experiment. Both $\mathrm{LHCb}$ and $\mathrm{BTeV}$ are fundametally different in design from the existing collider experiments - both are forward spectrometers (LHCb will have one arm, BTeV two) using a dipole magnet. Both should be able to measure all three angles of the unitarity triangle and so overconstrain the Standard Model description of CP violation.

\subsubsection{Current results}

Including the constraints caused by unitarity (and assuming three generations), the magnitudes of the CKM elements at the $90 \%$ confidence level are [12]

$$
\left(\begin{array}{ccc}
0.9742 \text { to } 0.9757 & 0.219 \text { to } 0.226 & 0.002 \text { to } 0.005 \\
0.219 \text { to } 0.225 & 0.9734 \text { to } 0.9749 & 0.037 \text { to } 0.043 \\
0.004 \text { to } 0.014 & 0.035 \text { to } 0.043 & 0.9990 \text { to } 0.9993
\end{array}\right)
$$

The CP violating effects in the CKM matrix can be described by the unitarity triangle (figure 2.1). Experimental results for the angle $\beta$ are shown in table 2.2. All come from the asymmetry in $B^{0} \rightarrow J / \psi K_{S}$ decays. Figure 2.7 shows a global fit in the $(\bar{\rho}, \bar{\eta})$ plane showing the constraints on the unitarity triangle. The experimental results so far are compatible with the Standard Model description of CP violation and show no sign of new physics. 


\begin{tabular}{|c|c|c|}
\hline Experiment & Date & $\sin 2 \beta$ (errors are stat. and syst.) \\
\hline Opal [34] & 1998 & $3.2_{-2.0}^{+1.8} \pm 0.5$ \\
CDF [8] & 1999 & $0.79 \pm 0.39 \pm 0.16$ \\
Belle [10,35] & 2001 & $0.99 \pm 0.14 \pm 0.06$ \\
BABAR [9,36] & 2001 & $0.59 \pm 0.14 \pm 0.05$ \\
\hline
\end{tabular}

Table 2.2: Experimental results for $\sin 2 \beta$

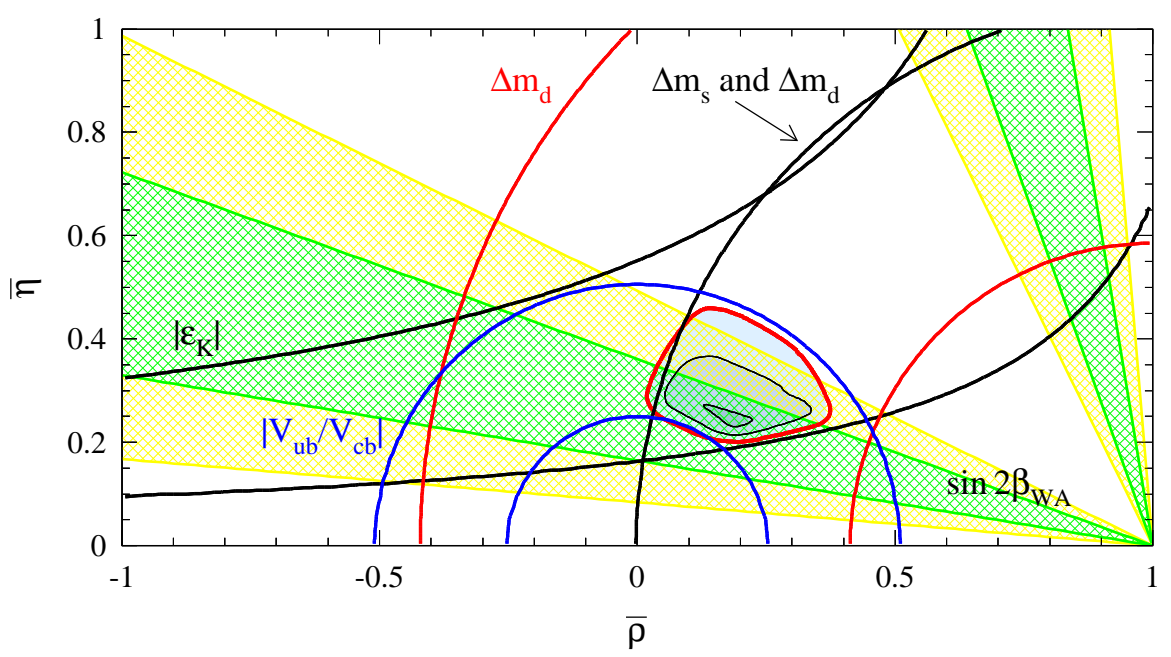

Figure 2.7: Global fit for $(\bar{\rho}, \bar{\eta})$ including initial $\sin 2 \beta$ results from BABAR and Belle (from [37])

\subsubsection{CP violation measurements with the $\mathrm{D} \varnothing$ detector}

With no central magnetic field, the Run I D $\varnothing$ detector was unable to study $B$ mesons in any detail. The added capabilities of the Run II upgrade will allow the experiment to measure $\sin 2 \beta$ to comparable accuracy to the $B$ factories.

The key requirement of doing $B$ physics at a hadron collider is to be able to trigger on the events. The large coverage of the muon trigger (which can match muons to tracks in the central region) provides a good trigger for $B \rightarrow J / \psi \rightarrow \mu^{+} \mu^{-}$decays $^{2}$. The electron identification abilities of the calorimeter and preshower detectors provide a trigger on $B \rightarrow J / \psi \rightarrow e^{+} e^{-}$decays. It is also conceivable to have single lepton

\footnotetext{
${ }^{2}$ And on indirect decays through $J / \psi$, such as $B \rightarrow \psi(2 S) \rightarrow J / \psi \pi^{+} \pi^{-}$.
} 
triggers to catch decays such as $B^{0} \rightarrow \pi^{+} \pi^{-}$where the $\bar{B}^{0}$ decays semi-leptonically, but here the background rate will be extremely high.

Having triggered on an event, it is then necessary to reconstruct it and to extract the useful physics from it. Here the large coverage and high precision of the silicon vertex detector allows the accurate determination of the decay length of the $B$, and the large coverage of the muon system and calorimeter provide a good method of tagging the initial flavours of the $b$ quarks, both of which are important requirements for measuring $\sin 2 \beta$ from the decay $B^{0} \rightarrow J / \psi K_{S}$, and for measuring flavour mixing in $B^{0} \bar{B}^{0}$ systems.

Chapter 3 gives a description of these, and other, features of the D $\varnothing$ detector. Chapter 7 contains the results of a study on Monte Carlo simulated data of reconstructing $B^{+}$mesons, and on measuring the lifetime of the $B^{+}$. Finally, section 8.1 explains how $\sin 2 \beta$ will be measured at $\mathrm{D} \varnothing$. 


\section{Chapter 3}

\section{The DØ detector}

\subsection{Overview of the upgraded detector}

The $D \varnothing$ detector has the typical, layered, design of most modern high energy physics experiments. The beam pipe is surrounded by concentric layers of different subdetectors, and, as both the proton and antiproton beams in the Tevatron have the same energy, the detector is approximately symmetric in each direction along the beam pipe.

Figure 3.1 shows a side view of the detector. The very central region contains the precision silicon vertex detector, the fibre tracker, a 2 Tesla solenoid, and the central and forward preshower detectors. Surrounding this are the cryostats containing the calorimeter, and the outermost sections are the toroid magnets, scintillator planes, and drift chambers which perform the muon detection. Each of these subdetectors is described in the following sections.

\section{A note on sources}

A full description of the upgraded $D \varnothing$ detector has not yet been published. The description of the Run I detector can be found in reference [2]. A 1996 description 
of the planned upgrade is in [3], and a 1998 one in [4], but there were a significant number of changes between these and completion of the detector. Reference [5] is a more recent, but brief, summary. The conceptual design report for the Run IIb trigger upgrade [38] also describes the Run IIa trigger systems. The contents of this chapter are derived from the above references, and from internal documents and personal conversations.

\subsection{Central tracking}

The tracking system (figure 3.2) consists of an inner silicon microstrip tracker (SMT) which is surrounded by the central scintillating fibre tracker $(\mathrm{CFT})$. These are placed within a 2 Tesla superconducting solenoid, and surrounding this is a scintillator based preshower detector. There are also preshower detectors mounted on the inner faces of the forward calorimeter cryostats. The tracking system is designed to provide momentum measurement from the magnetic field; good electron identification and $e / \pi$ rejection; tracking over a large pseudorapidity range $(|\eta| \lesssim 3)$; secondary vertex detection for top and $b$ physics; triggering at all levels; and a fast response for a bunch crossing time of 396 ns.

\subsubsection{Solenoid}

The solenoid is $2.8 \mathrm{~m}$ long and has a mean radius of $60 \mathrm{~cm}$ [4]. It is superconducting with a field strength of $2 \mathrm{~T}$, and has a stored energy of $5 \mathrm{MJ}$. Inside the tracking volume the value of $\sin \theta \times \int B_{z} d l$ is uniform to within $0.5 \%$. Due to the lack of a flux return this uniformity is achieved by using two grades of conductor in the solenoid to shape the field. The solenoid coil and its cryostat are together about 1.1 radiation lengths thick. 


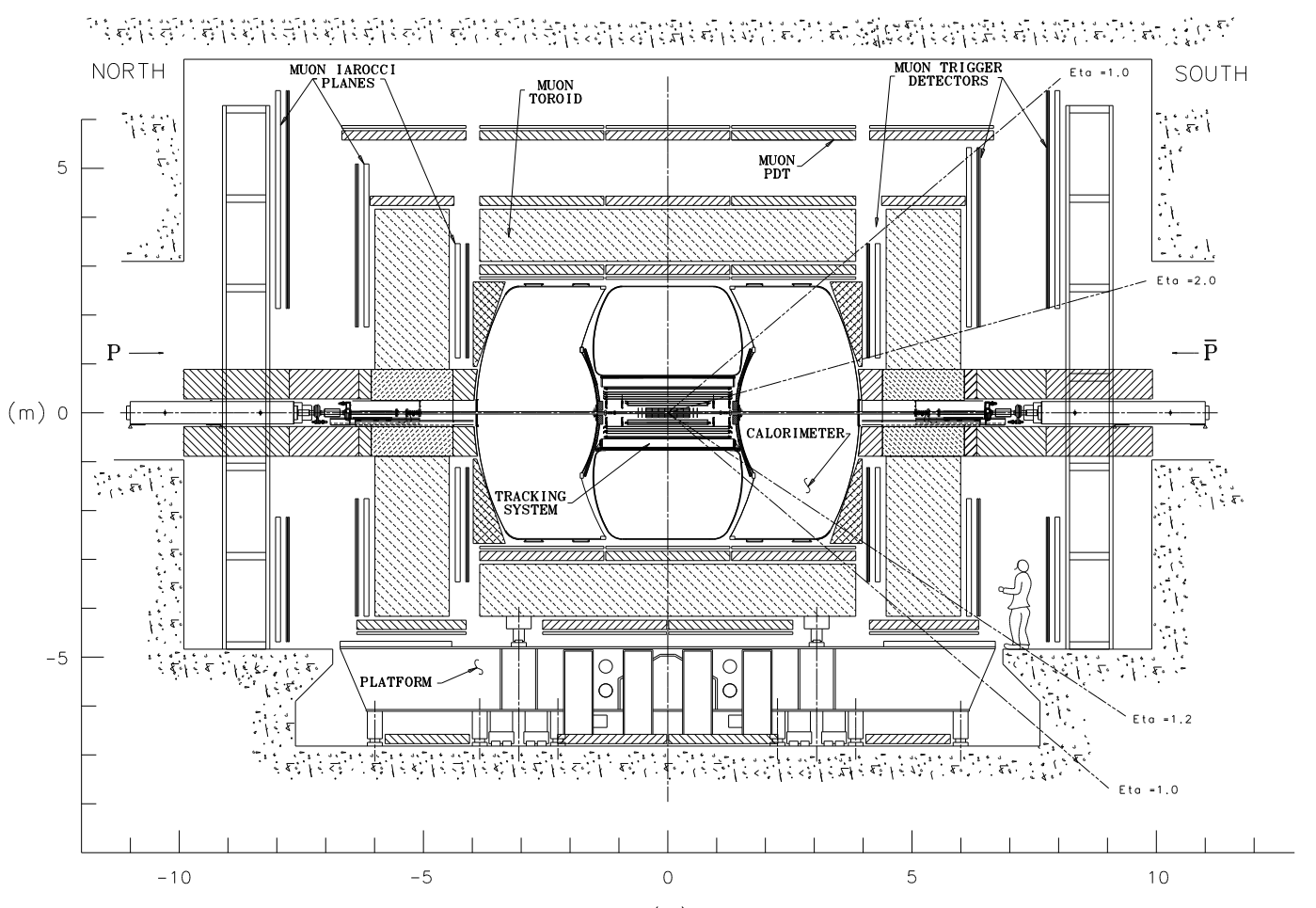

$(m)$

Figure 3.1: Side view of the upgraded detector 


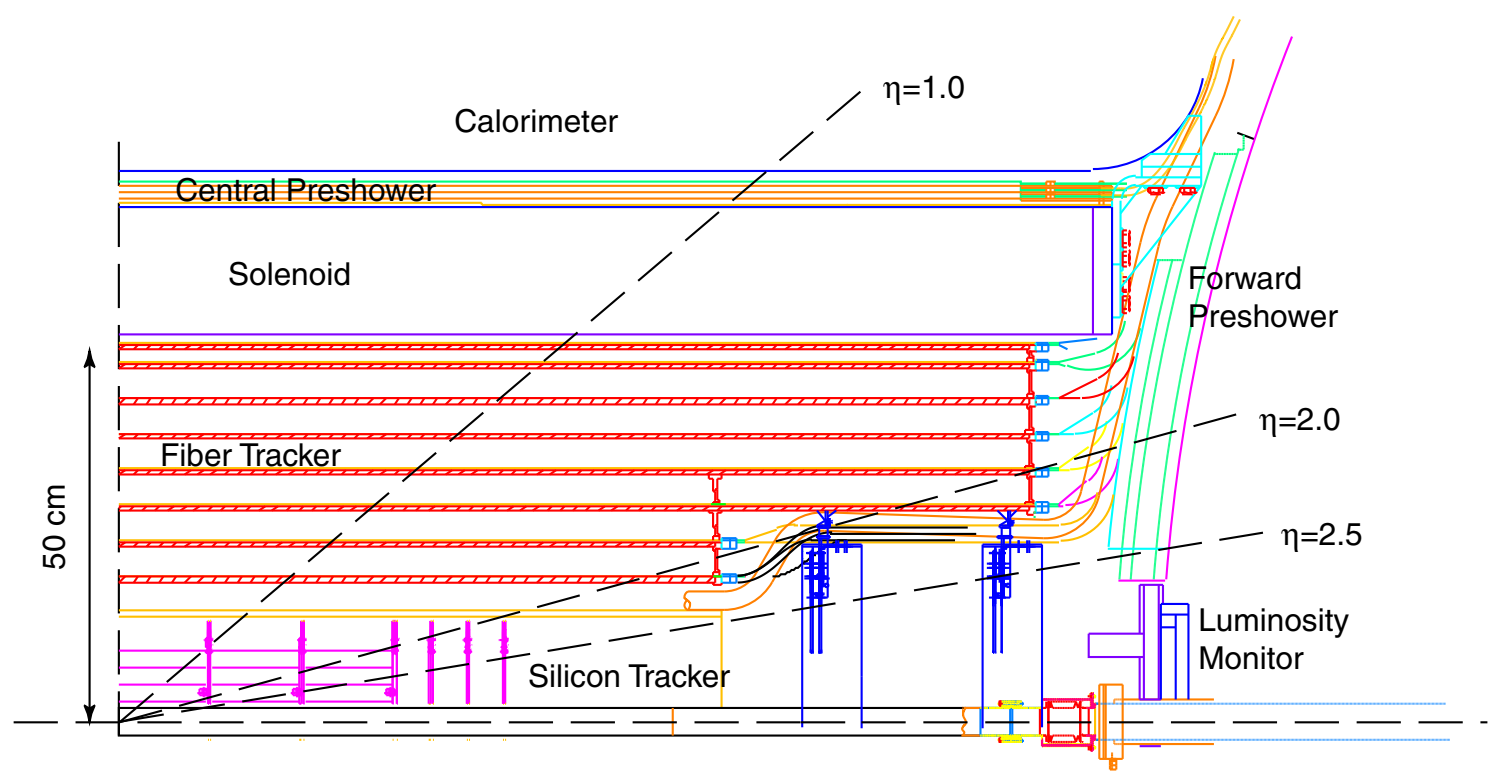

Figure 3.2: The tracking system

\subsubsection{Silicon Microstrip Tracker}

The silicon tracker $[39,40]$ is the first detector encountered by the particles after the collision and provides the highest resolution tracking. The design must cope with the extended luminous region of the collider, which is a Gaussian distribution with $\sigma_{z}=25 \mathrm{~cm}$. The detector consists of barrel shaped elements with interleaved disks positioned perpendicular to the beam pipe (figure 3.3).

Each of the six barrel segments is $12 \mathrm{~cm}$ in length. A barrel has eight layers of silicon strip detectors. In the inner four barrels, layers one, two, five, and six are double sided with axial strips on one side and $90^{\circ}$ stereo angle strips on the other side, with pitches of $50 \mu \mathrm{m}$ and $153.5 \mu \mathrm{m}$ respectively. The outer barrels have single sided detectors with $50 \mu \mathrm{m}$ pitch axial strips in these layers. In all the barrels, layers three, four, seven, and eight are double sided with axial strips of $50 \mu \mathrm{m}$ pitch on one side and $2^{\circ}$ stereo angle, $62.5 \mu \mathrm{m}$ pitch strips on the reverse side. The innermost layer is at $2.7 \mathrm{~cm}$ radius and the outermost is at $9.7 \mathrm{~cm}$ radius.

There are a total of twelve disks shaped detectors (' $\mathrm{F}$ disks') positioned between the barrel segments (except at the very centre) and spaced out beyond (see figures 


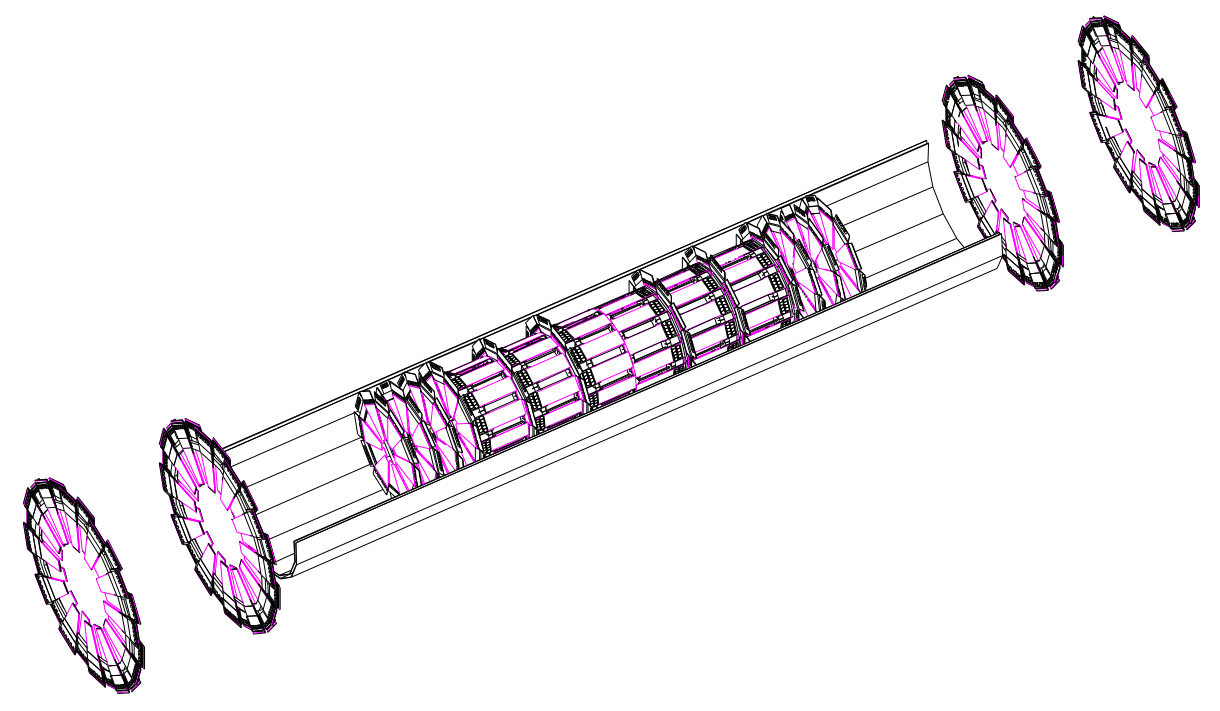

Figure 3.3: The Silicon Microstrip Tracker (SMT)

3.2 and 3.3). Each $\mathrm{F}$ disk has 12 wedge shaped double sided detectors with $50 \mu \mathrm{m}$ pitch, $-15^{\circ}$ stereo angle on one side and $62.5 \mu \mathrm{m}$ pitch, $15^{\circ}$ stereo angle on the other. Their active area lies between $2.5 \mathrm{~cm}<r<9.8 \mathrm{~cm}$.

Positioned some distance beyond the ends of the main body of the tracker are larger disks ('H disks') consisting of back to back pairs of single sided detectors with $80 \mu \mathrm{m}$ pitch and $\pm 7.5^{\circ}$ stereo angles. Their active area is between $9.6 \mathrm{~cm}<r<23.6 \mathrm{~cm}$.

The readout electronics of the silicon detector is described in section 4.1.

\subsubsection{Central fibre tracker}

The central fibre tracker [41] surrounds the silicon detector and covers the central pseudorapidity region $(|\eta|<2)$. Its purpose is track reconstruction and momentum measurement for charged particles within this region, and also to provide a fast level 1 trigger.

The basic element of the tracker is the scintillating fibre, consisting of a polystyrene core clad in a thin acrylic layer and a thin layer of fluoroacrylate [4]. The purpose of the claddings is to improve the light trapping and mechanical strength of the fibre. 
The nominal diameter of the clad fibres is $830 \mu \mathrm{m}$. The polystyrene core is doped to cause it to scintillate in the yellow-green part of the visible spectrum.

The fibres are formed into doublet layers consisting of a row of fibres with a second row above it offset by a fibre radius to lie between the fibres in the lower row. This supplies complete coverage, with no gaps between fibres.

The doublet layers are placed on eight supporting cylinders, arranged in concentric layers between $r=20 \mathrm{~cm}$ and $r=50 \mathrm{~cm}$ (see figure 3.2). Each cylinder has an axial fibre doublet layer and a doublet layer with a $\pm 2^{\circ}$ stereo angle. The stereo layers on alternate cylinders are in oppositely oriented directions. The tracker contains a total of 76800 fibres.

The fibres are connected by $7-11 \mathrm{~m}$ long optical waveguides to photodetectors positioned in a cryostat underneath the central calorimeter. The detectors used are visible light photon counters (VLPCs), which are arsenic doped silicon diodes operating at temperatures of $8-10 \mathrm{~K}$. The VLPCs have quantum efficiencies of $\sim 80 \%$, high gain and less than $0.1 \%$ average noise. In cosmic ray tests the hit efficiency for the doublet layers was better than $99.9 \%$ and a position resolution of $\sim 92 \mu \mathrm{m}$ was obtained.

The axial fibre layers are used in the Level 1 trigger system. In the event of a Level 1 trigger accept, the whole fibre system (axial and stereo) is read out and is available for use in the Level 3 trigger.

\subsubsection{Preshower detectors}

The preshower detectors aid electron identification and triggering and the central preshower also corrects for electromagnetic cascades in the solenoid. The central preshower fits in the $51 \mathrm{~mm}$ gap between the solenoid coil and the calorimeter cryostat wall. The forward preshowers are positioned on the inner faces of the forward calorimeter cryostats. 
The central preshower covers the region $|\eta|<1.2$ and consists of three layers of scintillating strips: one axial layer and two stereo layers at $\pm 23^{\circ}$ stereo angles. The strips have a triangular cross section with a base of $\sim 7 \mathrm{~mm}$. A wavelength shifting fibre passing through a hole in the centre of the strip is used for the readout. The light is transmitted by clear waveguides and is readout by VLPCs in the same way as the fibre tracker.

In front of the detector there is a flat layer of lead, $5.5 \mathrm{~mm}$ thick (approximately one radiation length), to act as a preradiator.

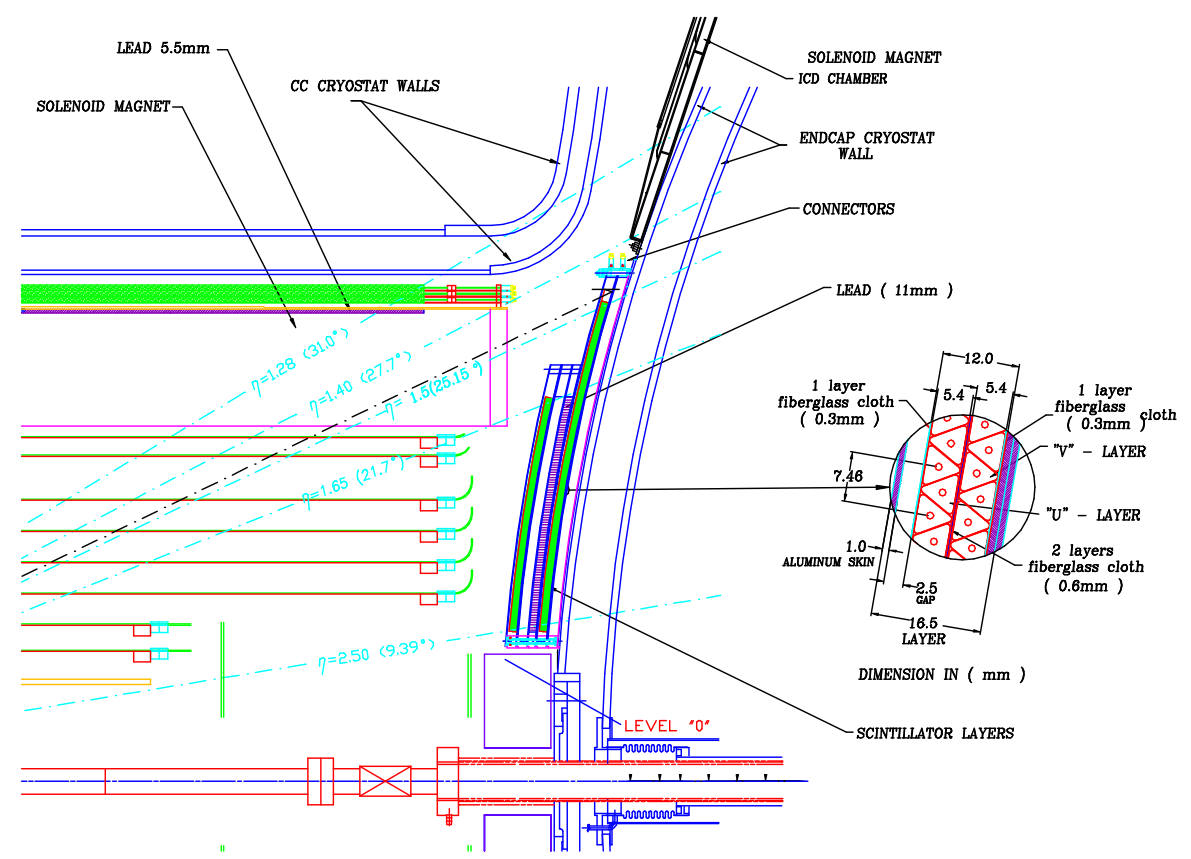

Figure 3.4: The forward preshower detector

The forward preshower covers the region $1.4<|\eta|<2.5$ (see figure 3.4). It is of similar design to the central preshower, using scintillating strips. In the most forward region $(1.6<|\eta|<2.5)$ the particles pass clear of the solenoid and the detector consists of a layer of lead $11 \mathrm{~mm}$ thick (approximately 2 radiation lengths) sandwiched between two scintillator planes. The purpose of the first layer is to detect minimum ionizing particles. In the range $1.4<|\eta|<1.6$ there is only one scintillator plane and no lead layer (particles in this region pass through a significant 
thickness of the solenoid). Each scintillator plane in the forward preshowers consists of two layers of triangular strips of the same type as used in the central preshower, with stereo angles of $\pm 23^{\circ}$.

\subsection{Calorimeter}

The calorimeter itself has not been altered in the upgrade, although its supporting electronics have been replaced to cope with the new, much shorter bunch crossing time of 396 ns (132 ns in Run IIb).

The calorimeter, shown in figure 3.5, is a liquid argon sampling calorimeter using depleted uranium, copper and stainless steel absorbers [2]. The depleted uranium provides some compensation $^{1}$, with the ratio of the electromagnetic to the hadronic response ranging from 1.11 at $10 \mathrm{GeV}$ to 1.04 at $150 \mathrm{GeV}$. The use of liquid argon makes the design inherently resistant to radiation damage.

\subsubsection{Central calorimeter}

The central calorimeter is divided into three sections, comprising cylindrical shells around the beam pipe. The innermost, centre, and outermost sections are, respectively, the electromagnetic $(\mathrm{EM})$, fine hadronic $(\mathrm{FH})$, and coarse hadronic $(\mathrm{CH})$ regions. Each region is subdivided into a number of layers, and segmented in both the $\phi$ and $z$ coordinates. Each segmented readout cell comprises a thickness of absorber and a gap filled with liquid argon (see table 3.1). The combined sections are 6.9 absorption lengths thick. The segmentation is approximately $0.1 \times 0.1 \mathrm{in}$

${ }^{1} \mathrm{~A}$ compensating calorimeter is one where the calorimeter response to electromagnetic activity and hadronic activity is equal. Usually the electromagnetic response is greater than the hadronic response because much of the low-energy hadronic component is 'hidden' in nuclear binding energy release, etc. Compensation is a desirable feature of a calorimeter because a hadronic shower contains an electromagnetic component from $\pi^{0}$ decays, so a non-compensating calorimeter suffers from problems including an energy dependent response ratio for electrons and hadrons and a non-linear response to hadrons. 
]

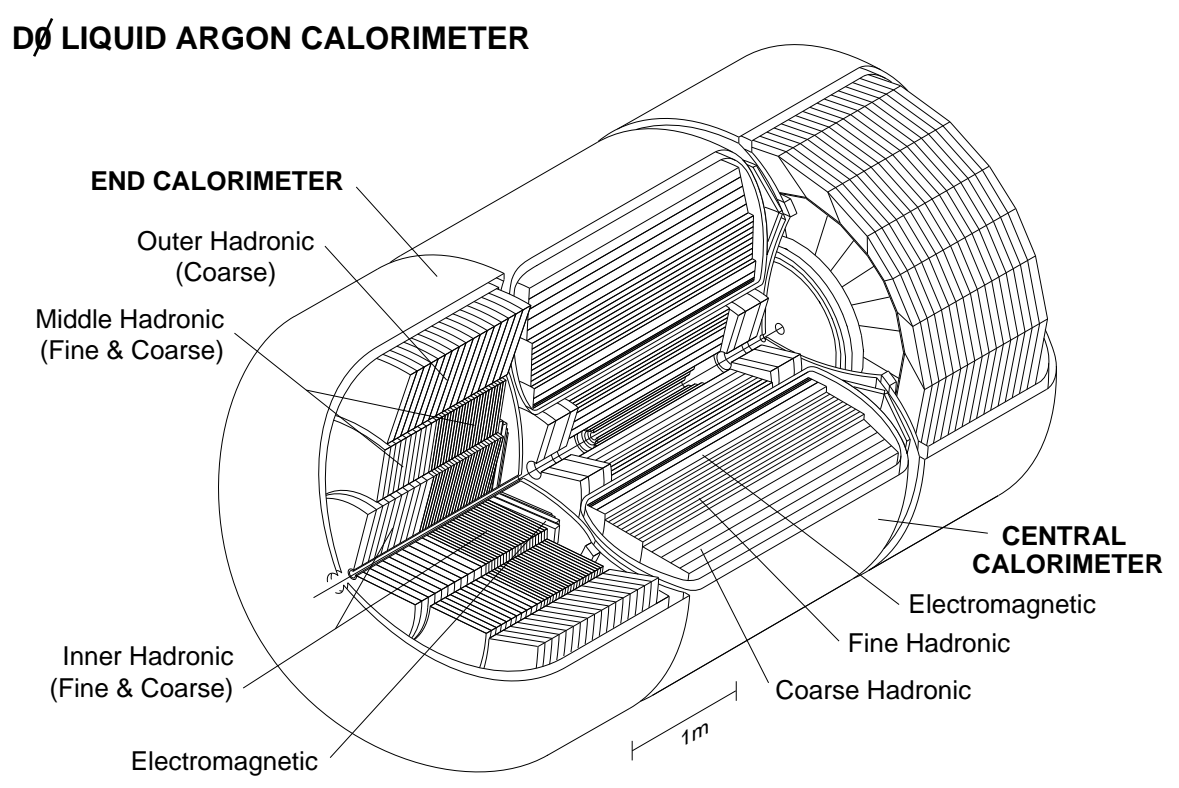

Figure 3.5: The calorimeter. (The centre of this picture actually shows the Run I tracking detector rather than the Run II tracker.)

$\eta \times \phi$ space, except for the third readout layer of the electromagnetic region, which is segmented as $0.05 \times 0.05$ to improve electron/hadron shower identification.

\subsubsection{End calorimeter}

The end calorimeters are divided into four sections: the forward electromagnetic (EM), the inner hadronic (IH), the middle hadronic $(\mathrm{MH})$, and the outer hadronic $(\mathrm{OH})$. The inner and middle hadronic sections are additionally split into fine and coarse sections (see table 3.2). The end calorimeter segmentation is $\Delta \phi=\Delta \eta=0.1$, except for $|\eta|<2.6$ in layer three of the EM region where $\Delta \phi=\Delta \eta=0.05$, and for $|\eta|>3.2$ in all the sections, where $\Delta \phi=0.2$, and $\Delta \eta$ becomes larger as well. 
Table 3.1: Central calorimeter parameters (see text for key)

\begin{tabular}{|l|ccc|}
\hline & EM & FH & CH \\
\hline Rapidity coverage & \pm 1.2 & \pm 1.0 & \pm 0.6 \\
Absorber & Uranium & Uranium & Copper \\
Absorber thickness (mm) & 3 & 6 & 46.5 \\
Argon gap (mm) & 2.3 & 2.3 & 2.3 \\
Number of readout layers & 4 & 3 & 1 \\
Total radiation lengths & 20.5 & 96.0 & 32.9 \\
Total absorption lengths & 0.76 & 3.2 & 2.9 \\
Sampling fraction & $11.8 \%$ & $6.8 \%$ & $1.5 \%$ \\
Readout channels & 10368 & 3456 & 768 \\
\hline
\end{tabular}

Table 3.2: End calorimeter parameters

\begin{tabular}{|l|cccccc|}
\hline & $\mathrm{EM}$ & $\mathrm{IFH}$ & $\mathrm{ICH}$ & $\mathrm{MFH}$ & $\mathrm{MCH}$ & $\mathrm{OH}$ \\
\hline Rapidity coverage & $1.3-4.1$ & $1.6-4.5$ & $2.0-4.5$ & $1.0-1.7$ & $1.3-2.0$ & $0.7-1.4$ \\
Absorber & $\mathrm{U}$ & $\mathrm{U}$ & $\mathrm{SS}^{\dagger}$ & $\mathrm{U}$ & $\mathrm{SS}$ & $\mathrm{SS}$ \\
Absorber thickness (mm) & 4 & 6 & 46 & 6 & 46 & 46 \\
Argon gap (mm) & 2.3 & 2.1 & 2.1 & 2.2 & 2.2 & 2.2 \\
Number of readout layers & 4 & 4 & 1 & 4 & 1 & 3 \\
Total radiation lengths & 20.5 & 121.8 & 32.8 & 115.5 & 37.9 & 65.1 \\
Total absorption lengths & 0.95 & 4.9 & 3.6 & 4.0 & 4.1 & 7.0 \\
Sampling fraction & $11.9 \%$ & $5.7 \%$ & $1.5 \%$ & $6.7 \%$ & $1.6 \%$ & $1.6 \%$ \\
Readout channels & 14976 & 8576 & 1856 & 2944 & 768 & 1784 \\
\hline
\end{tabular}

$\dagger$ Stainless steel

\subsubsection{Intercryostat detector}

In the crossover region from the central to the end calorimeter, there are several regions where particles travel mostly through support structures (such as the cryostat walls). To compensate for the energy losses in these gaps there are extra sampling layers on the end faces of the central calorimeter and on the inner faces of the end cryostats, and there is also an intercryostat detector consisting of a single layer array of 384 scintillating tiles mounted on the face of both end cryostats. The tile size is chosen to match the calorimeter cell size. The scintillation light is taken by optical fibres to phototubes outside the magnetic field region. 


\subsection{Muon system}

The upgraded muon system is designed to provide coverage to $|\eta| \sim 2$ and efficient triggering to study low cross section, high $p_{\mathrm{T}}$ processes at high luminosity. The muon tracking uses toroid magnets with a field of $1.9 \mathrm{~T}$ to bend the muon trajectories in the $r z$ plane. The system is divided into the wide angle muon system (WAMUS) covering the central region $(|\eta|<1)$, and the forward muon systems (FAMUS) covering $1<|\eta|<2$.

\subsubsection{Wide angle muon system}

The WAMUS region consists of three layers of drift chambers, with one of the layers inside and the other two outside the toroid magnets. The purpose is to provide muon identification and to provide a confirming momentum measurement, although the resolution is dominated by the central tracking. The chambers are extruded aluminium tubes with a rectangular cross section. The wires are $50 \mu \mathrm{m}$ gold clad, nickel struck tungsten. The hit uncertainty due to diffusion is about $375 \mu \mathrm{m}$.

In addition to the drift tubes there are layers of scintillation counters, which provide a fast signal for use in the trigger, and also because the drift time in the tubes (500 ns) is greater than the beam crossing time (down to $132 \mathrm{~ns}$ ). The scintillators have a time resolution of $1.6 \mathrm{~ns}$ and can be used to reject out-of-time backgrounds in the drift tubes. There is a layer of counters between the first drift tube layer and the calorimeter, and one outside of the outer drift tube layer, except for the underside, where some of the counters are positioned on the outside of the middle drift tube layer due to the positioning of the support structures for the calorimeter. There are a total of 986 counters overall. 


\subsubsection{Forward muon system}

The forward muon system uses plastic mini-drift proportional tubes for track reconstruction and scintillation counters for triggering.

The mini drift tubes are $10 \mathrm{~mm} \times 10 \mathrm{~mm}$ internal cross section with $50 \mu \mathrm{m}$ anode wires in the centre of the cell. The cells are extruded in packs of eight and are up to 5 m long. The internal surfaces are covered with conductive paint to form the cathode. The tubes are oriented along the magnetic flux lines. The planes are arranged in three layers, one with 4 planes inside the toroid and two layers each with three planes outside of the toroid. In the forward region the central tracking momentum resolution falls off and the contribution to the resolution from the drift tubes become important. The coordinate resolution from the drift tubes is $\sim 0.8 \mathrm{~mm}$, and the limiting factor for momentum resolution will be multiple scattering rather than the position measurements.

The drift time in the mini drift tubes is $60 \mathrm{~ns}$, less than the beam crossing time, and so scintillation counters are not needed to match drift tube hits to events. However the forward scintillation counters are still useful for reducing backgrounds coming from sources other than the interaction region (such as cosmic rays). There are three layers of scintillation counters, corresponding to the drift tube layers. The layers are segmented with $\Delta \phi=4.5^{\circ}$ and $\Delta \eta=0.1$, values chosen to trigger on low momentum muons with multiple scattering up to $3^{\circ}$, but which are not so coarse as to give a high false trigger rate on combinatoric backgrounds.

\subsection{Triggering}

The upgraded trigger has three levels. Level 1 is fully hardware based, using Field Programmable Gate Array (FPGA) microchips, Level 2 uses both FPGAs and microprocessor cards, and Level 3 runs on a farm of standard computers. 
The output rates and times required for the different levels are shown in the table below (the time required for a Level 1 decision is constant, for Level 2 and 3 it is variable and the values are the upper limits).

\begin{tabular}{ccc}
\hline & Rate & Latency \\
\hline Events & $7.6 \mathrm{MHz}$ & \\
Level 1 & $6 \mathrm{kHz}$ & $3.6 \mu \mathrm{s}$ \\
Level 2 & $1 \mathrm{kHz}$ & $100 \mu \mathrm{s}$ \\
Level 3 & $20-50 \mathrm{~Hz}$ & $150 \mathrm{~ms}$ \\
\hline
\end{tabular}

The limiting factor on the final output rate is the cost of magnetic tape to store the data on. A $20 \mathrm{~Hz}$ rate of recorded events corresponds to around $300 \mathrm{~TB}$ of data per year. If the cost of storage falls significantly then the triggering and data acquisition are both easily upgradable to higher rates.

\subsubsection{Level 1 trigger}

The Level 1 trigger [42] uses the calorimeter, the central fibre tracker (axial fibres only), the preshowers, and the muon scintillators and drift tubes. In each case a reduced form of the detector readout is used. For the calorimeter, the analogue readout is digitised by a separate circuit from the main readout, and the separate cell energies are summed to form trigger towers, which are used to form the trigger decision. The muon subsystem digitises the signal on every bunch crossing, but only uses discriminated (on/off) signals for each channel at Level 1. The fibre tracker and preshower (which share their readout electronics) form a discriminated value for each fibre using the analogue signal. The responses from the separate detector processors are logically combined into up to 128 specific triggers. The output state of each of the triggers is called a trigger bit - the bit is said to be set if the event is passed by that particular trigger logic. The trigger decision is made $3.6 \mu$ s after the beam crossing. Every subdetector has buffers to hold the data from sixteen events while waiting for the Level 1 trigger decision. If any one of the triggers is accepted 
the event data is digitised for those subdetectors which have not yet done so and sent into one of eight buffers to await the Level 2 trigger decision.

\subsubsection{Level 2 trigger}

The Level 2 trigger [42] combines and refines the outputs from the Level 1 trigger. Each of the 128 Level 1 trigger bits is associated with one or more Level 2 trigger algorithms, and the event is processed by all the algorithms matching the set Level 1 bits.

The Level 2 trigger hardware consists of a framework of FPGA logic chips to coordinate the operation of the trigger, and VME cards containing a Digital Alpha based microprocessor to perform the processing. There are preprocessor cards for the muon system, the calorimeter, the preshower detectors, and central tracking (again only using the axial fibres). The preprocessors transform the Level 1 trigger information into physics objects (tracks, energy clusters, etc.) and pass these into a global processor which is responsible for correlating the outputs from the different detector elements. Reducing the data to the physics objects allows the transfer to the global processor to be done much more quickly than if raw data was sent, due to the small size of the objects. If the Level 2 trigger passes the event then the full detector is read out, and the data sent to the Level 3 trigger. The Level 2 decision is made within $100 \mu \mathrm{s}$, and at the highest expected data rates the deadtime is $5 \%$ or less.

An extra preprocessor for a silicon track trigger is being designed. This will use the silicon vertex detector to look for tracks displaced from the primary vertex, and to improve the $p_{T}$ threshold for the Level 2 track trigger. 


\subsubsection{Level 3 trigger}

The Level 3 trigger is a software based, fully programmable trigger that refines the physics objects created by the Level 2 trigger and performs a limited reconstruction of the whole event. It will have up to $150 \mathrm{~ms}$ to decide on an event, and given the expected input rate of $1 \mathrm{kHz}$ and an output rate of $20 \mathrm{~Hz}$ it will have to pass about 1 event in 50 .

The event filtering software runs on a farm of standard PC computers running the Linux operating system. Each processor of the farm (a 'Level 3 filter node') runs an independent instance of the Level 3 filtering software and each instance sequentially processes complete events. The events are distributed among the nodes in order to balance the load between them. Events which are passed by the trigger are transmitted from the node on to the datalogger, from where they are sent to tape.

The trigger filters events based on complete physics objects (such as electrons, jets, muons, taus or missing $E_{T}$ ) and on the relationships between objects. Unlike the earlier trigger levels, the complete read out data of the detector is available to reconstruct these objects. The objects, and the relations between them, are generated by software algorithms called physics tools [43].

The trigger parameters are defined by filters. A filter script contains a list of the physics tools that are to be used to process the event, and the parameters to be passed to the tools. For consistency across different filters there will be a limited number of parameter sets (called reference sets, or refsets) for each physics tool: e.g. there will be only three refsets defining an electron, each with differently demanding selection cuts.

Filters come in two main types:

1. Physics object filters - these determine if the physics object candidates returned by the tools pass the trigger criteria. 
2. Relational filters - these combine candidates passed by one or more other filters into a composite object, such as an invariant mass.

The programming for each Level 3 trigger (a Level 3 filter bit) consists of one or more filters, collectively known as a filter script. The Level 3 filter bits are matched to Level 2 bits, and all the filter scripts for the corresponding Level 2 bit are run if the bit is set. There is no limit, except for available processing power, on the number of filter scripts for each Level 2 bit.

\section{Executing filter scripts}

The software interface to the physics tools is called ScriptRunner. It is responsible for executing the scripts and takes as its input the 'trigger list' of filter scripts and the event data.

When it is initialized at the start of a data-taking run ScriptRunner reads in the tool refsets, initializes the tools to be used, and then builds an execution tree of the scripts and filters. Assuming this is done without producing any fatal errors, it then signals it is ready to receive data.

Then, event by event, ScriptRunner follows the execution tree for each set Level 2 trigger bit. This means running, in order, the filters and tools chained below that trigger bit. Failure of any particular filter within a filter script ends the execution of the script at that point and begins the execution of the next filter script.

An event is passed by the trigger if all the filters for any one of the filter scripts pass. It would be possible for processing to stop at this point. However, most events fail, and all the relevant scripts must be run on them to do this. There is therefore little performance gain in stopping processing as soon as an event passes the trigger, and it simplifies timing analysis and luminosity calculations if it is not stopped. For this reason all the relevant filter scripts are always run on an event, even when it is accepted. 
Passed events are written out to the data logging system, along with status information on which scripts passed or failed.

As an additional control on the data rates, Level 3 filters can contain prescale factors. These reject a certain fraction of the events that would otherwise have been processed by the filter. This artificially limits the number of events accepted by the filter if it would otherwise become too high.

\section{Tools}

The tools are the algorithms that actually process the event data. Tools are called by filters, and tools can also call other tools. There are several different types:

1. Physics tools - these identify physics objects, like electrons, muons, taus, jets, and photons. The physics tools take as their input the Level 2 trigger candidate objects and do a more detailed reconstruction using the full event data.

2. Unpacking tools - these unpack the raw data from the detectors to put it into a form that can be accessed by the physics tools. The data can be unpacked selectively on demand, for example only one $\phi$ sector of the fibre tracker could be accessed if it is the only interesting region.

Chapters 4 and 5 contain details of the unpacking tools for the silicon and fibre trackers.

3. Trigger tools - these tools provide support functionality, such as error handling and rejecting events due to prescales.

\section{An example filter: $b$ tagging}

An example of a complex filter is one implementing an impact parameter $b$ quark tagger [44]. Its purpose is to identify events which contain jets which have a high 
probability of originating from the decay of a $b$ quark. Since the $b$ quark has a relatively long lifetime, the hadron that contains it may travel a significant distance (of the order of millimeters) before decaying. Tracks from the displaced vertex will have a large impact parameter (measured with respect to the primary vertex) compared to those from prompt decays.

The $b$ tag filter calls the $b$ tag physics tool. The tool then calls the jet, tracking, and primary vertex tools. If these tools have been run before for this event, they return their stored results, otherwise they carry out their processing. This involves calling other tools, until they eventually reach the raw data. For example the jet tool will call the calorimeter clustering tool, while the tracking tool will call the unpacking and clustering tool for the fibre tracker.

When the called tools have run, the $b$ tag tool is left with a list of jet candidates, a list of tracks, and the position of the primary vertex. The tool now does its own processing - it attempts to associate tracks with each jet, and for each jet of tracks uses the track impact parameters to calculate a probability that the jet is from a $b$ decay. Finally the list of $b$ jet candidates is returned to the filter. If one or more of the candidates meets the cuts specified in the filter reference set, then the filter is passed. If the rest of the filters in a filter script along with the $b$ tag filter also pass then the event has passed the trigger and will be written out to tape.

\subsection{Software}

Almost all of the code for the reconstruction and programmable triggers (Level 2 and 3 triggers) is written using the $\mathrm{C}++$ programming language. The framework for the reconstruction software [45] makes use of object-oriented programming techniques. The data for an event is stored in an Event object as a collection of 'chunks' of data (for example the raw data is stored in a RawDataChunk object). Each part of the reconstruction software (a framework package) communicates with the other parts only through the chunks - a package reads one or more existing chunks from the 
Event, executes its algorithm, and places the result as a new chunk back into the Event. As an example, a jet algorithm would read in a chunk containing calorimeter clusters, form the jets, store them in a JetChunk and insert that into the Event.

\subsection{Future upgrades}

The existing silicon detector is expected to be able to withstand radiation damage corresponding to an integrated luminosity of $2-4 \mathrm{fb}^{-1}$. This is enough for it to be able to survive the whole of Run IIa, but will certainly not be sufficient for Run IIb, which would bring the total integrated luminosity up to at least $15 \mathrm{fb}^{-1}$. Additionally the increased instantaneous luminosity required for Run IIb will be too much for the existing trigger design to cope with. For this reason the detector will be further upgraded for Run IIb, with the complete replacement of the silicon detector [46] and its readout electronics, and further upgrades to much of the trigger electronics and hardware [38]. 


\section{Chapter 4}

\section{Silicon Microstrip Tracker readout software}

\subsection{SMT electronics and readout}

The silicon detectors are read out by SVXII chips. The SVXIIe [47] is a 128 channel, 8-bit silicon strip readout chip. Between three and nine chips are used per sensor. The different designs of sensor are summarised in table 4.1 [40].

Figure 4.1 shows a simplified view of the readout chain [48] from the ladders to the front end crates. The SVX chips for each detector element are mounted on a single high density interconnect (HDI), with between three and nine chips per HDI. On the barrels the chips for both sides of the double sided detectors are mounted on the same HDI, while for the F disks the two sides are on different HDIs. From the HDIs the data passes through the adaptor cards and the interface boards (omitted from the diagram) and then to the sequencer boards. The sequencers transmit the signal onwards using a fibre optic link. The optical signal goes to the VRB (VME readout buffer) boards in the front end crates, with a total of 8 HDIs connected to each VRB. Each crate also has a buffer driver (VBD) card which is responsible for 


\begin{tabular}{|l|c|c|c|c|c|}
\hline Sensor type & View & $\begin{array}{c}\text { No. of } \\
\text { chips }\end{array}$ & $\begin{array}{c}\text { No. of } \\
\text { strips }\end{array}$ & Stereo & Pitch $(\mu \mathrm{m})$ \\
\hline Barrel single sided & 1 & 3 & 384 & $0^{\circ}$ & 50 \\
Barrel $90^{\circ}$ & 1 & 3 & 384 & $0^{\circ}$ & 50 \\
& 2 & 3 & $768^{\dagger}$ & $90^{\circ}$ & 153.5 \\
Barrel $+2^{\circ}$ & 1 & 5 & 640 & $0^{\circ}$ & 50 \\
& 2 & 4 & 512 & $+2.004^{\circ}$ & 62.5 \\
Barrel $-2^{\circ \dagger \dagger}$ & 1 & 5 & 640 & $0^{\circ}$ & 50 \\
F disk & 2 & 4 & 512 & $-2.004^{\circ}$ & 62.5 \\
& 1 & 8 & 1024 & $-15^{\circ}$ & 50 \\
H disk & 2 & 6 & 768 & $15^{\circ}$ & 62.5 \\
& 1 & 6 & 768 & $7.5^{\circ}$ & 79.3 \\
\hline
\end{tabular}

$\uparrow$ These ladders are multiplexed, with two strips connected to one channel t† These ladders are installed upside down, so that the stereo strips are oriented in the same direction as on the $+2^{\circ}$ ladders

Table 4.1: SMT sensor parameters

collecting the data from the VRBs and sending it onwards to the Level 3 trigger nodes, and a controller (VRBC) card which manages the operation of the crate.

\subsection{Raw data format}

The VBDs are used in the readout of all D $\varnothing$ subsystems, and so there is a common interface to the contents of each readout crate. The unpacking code for each subsystem is presented with the raw data already broken into separate VRBs and does not need to deal with the header information added by the VBD.

The data format for the SMT has three main elements, corresponding to the major components of the readout system:

1. VRB header

2. HDI header/trailer (actually added by the sequencer, rather than the HDI)

3. SVX data 


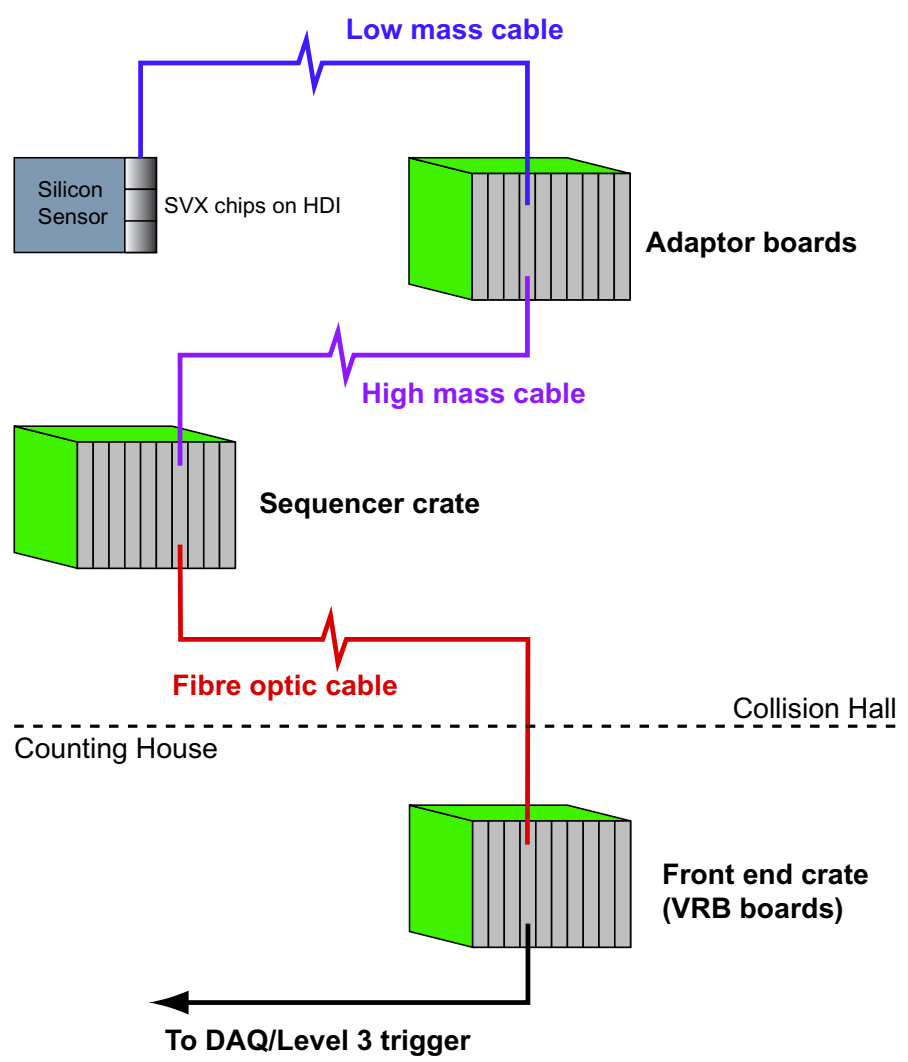

Figure 4.1: Diagram of the SMT readout system

\subsubsection{VRB header}

The header for the VRB is 16 words $^{1}$ long [51].

\begin{tabular}{|c|c|c|}
\hline \multicolumn{2}{|c|}{16} & \multicolumn{2}{c|}{} \\
\hline \multicolumn{2}{|c|}{ VRB byte count } \\
\hline User info & Slot no & Event number \\
\hline Firmware version & \multicolumn{2}{|c|}{ Config info } \\
\hline Status (meaning to be decided) \\
\hline HDI 0 byte count & HDI 1 byte count \\
\hline HDI 2 byte count & HDI 3 byte count \\
\hline HDI 4 byte count & HDI 5 byte count \\
\hline HDI 6 byte count & HDI 7 byte count
\end{tabular}

${ }^{1}$ One word equals two bytes throughout. The VRB is big-endian [49] — in multibyte numbers the most significant byte comes first 
The VRB byte count is the size of the entire VRB data in bytes. The meaning of the Status word is currently unassigned. The bits of the Config info byte have the following meaning:

\begin{tabular}{|c|c|}
\hline Bit & Meaning \\
\hline $7-2$ & Unassigned \\
1 & Trigger mode flag (always unset for SMT) \\
0 & Gray decode mode flag (always set for SMT — see page 66 ) \\
\hline
\end{tabular}

The byte count for an HDI is the size of all the data for this HDI including the header and trailer words. If an HDI is disabled its size is set to zero. Any extra data that appears between HDIs is not included in this total.

\subsubsection{HDI data}

The sequencer adds a one word header to the beginning of the data from each HDI. This is then followed by the SVX data for all of the chips in the HDI. Each chip adds its own header word and follows it with all the data from that chip. The SVX has the ability to operate in 'sparse mode', so that only channels above a preset threshold are read out. The number of channels in the data for each chip can therefore be less than the 128 channels supported by the SVX. After the end of the data for the last chip the sequencer adds a one word end of record marker. The data format therefore looks like this:

HDI header

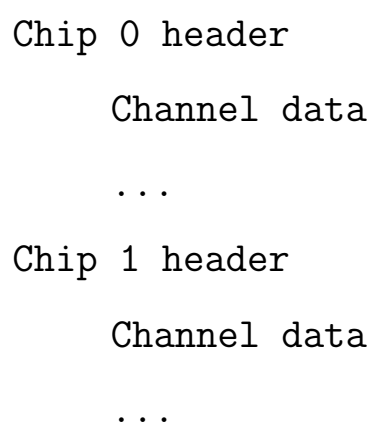


Chip $n$ header

Channel data

HDI end of record

\section{HDI header}

The HDI header contains the sequencer ID and the status and ID of the HDI [52].

\begin{tabular}{|llllllllllllllll|}
15 & 14 & 13 & 12 & 11 & 10 & 9 & 8 & 7 & 6 & 5 & 4 & 3 & 2 & 1 & 0 \\
\hline \multicolumn{8}{|c}{ sequencer } & ID & & & \multicolumn{1}{|c|}{ status } & & HDI & ID \\
\hline
\end{tabular}

\section{SVX data}

Each SVX data record consists of one word. There are two types of SVX data words - chip headers and channel data. The most significant bit indicates the type: if set, it signals a chip ID, otherwise it's an SVX channel.

The format of a chip ID word is:

\begin{tabular}{|l|lllllllllllllll|}
15 & 14 & 13 & 12 & 11 & 10 & 9 & 8 & 7 & 6 & 5 & 4 & 3 & 2 & 1 & 0 \\
\hline 1 & \multicolumn{1}{|c}{ chip ID } & & & \multicolumn{1}{|c|}{0} \\
\hline
\end{tabular}

The lower byte consists of all zeros ${ }^{2}$. 7 bits are provided for the chip ID, but by convention only the range $0-15$ is used.

The SVX channel data format is:

\begin{tabular}{|l|lllllllllllllll|}
15 & 14 & 13 & 12 & 11 & 10 & 9 & 8 & 7 & 6 & 5 & 4 & 3 & 2 & 1 & 0 \\
\hline 0 & \multicolumn{1}{|c}{ channel } & ID & & & \multicolumn{1}{|c}{ ADC count } & & \\
\hline
\end{tabular}

${ }^{2}$ This is true for genuine SVX chips, but not for the 'Virtual SVX' used in the CFT and preshower detectors. See Chapter 5 for details. 
The ADC counts produced by the SVX chip are in Gray code ${ }^{3}$. The VRB has the ability to convert the lower byte of each data word from Gray code into binary. This means that the data seen by the unpacking software is already in binary, and no further conversion is required. The VRB firmware knows not to apply Gray decoding to the VRB and HDI headers so these are left unaltered.

\section{HDI trailer}

At the end of the data from the HDI the sequencer adds a trailer word. This end of record marker is the hexadecimal word coco.

To allow for more efficient transfers over the VME bus the total size of the HDI data is rounded up to a multiple of eight bytes. This is done by following the end of record marker with enough zero bytes to bring it up to the next eight byte boundary. These additional padding bytes are not included in the byte count for the HDI (although they are included in the byte count for the whole VRB).

\subsection{Unpacking software design}

The DØ software dealing with raw data breaks down into two main categories: unpacking raw data and packing Monte Carlo data to create simulated raw data. In addition the unpacking splits into offline ${ }^{4}$ and Level 3 trigger applications. The main difference between the offline and Level 3 code is the extra execution speed needed to meet the timing requirements of the trigger.

\footnotetext{
${ }^{3}$ Gray code [53] is an alternative method to binary of encoding a number with bits. In Gray code each sucessive number differs by only one bit from the former, so $0=00,1=01,2=11$, $3=10$, etc. The advantage is that binary can involve changing a large proportion of the output for certain numbers - as an example, going from 127 to 128 involves changing the state of all eight bits with binary - and using Gray code can reduce the complexity of the electronics design and the error rate.

${ }^{4}$ This is also used by SMT Examine to monitor the detector online, so calling it 'offline' is something of a misnomer.
} 


\subsubsection{Unpacking}

There are two stages to the unpacking process. First it is necessary to interpret the raw data format described in section 4.2. Then this must be turned into meaningful information for the SMT detector, such as the position in the detector and calibrated pulse height of a given strip. In the offline code the design mirrors this split, while in the Level 3 trigger the two stages are combined to increase the speed.

In both cases the starting point is the raw data presented in the form of a RawDataChunk [54] in the standard DØ Event Data Model (EDM) format. The RawDataChunk provides methods to access the data for each VRB individually as a simple array of numbers.

\subsubsection{Offline unpacking}

The offline unpacking code fits into the standard DØ unpacking framework [55]. First the RawDataChunk is converted to an UnpDataChunk, which contains the same data in a structured form, so that the VRBs, HDIs, and SVX channels are represented as objects within the chunk. Then the data contained in the UnpDataChunk is calibrated and converted to the detector strip numbering system [56] and then put into the SmtDataChunk. This process is shown in figure 4.2

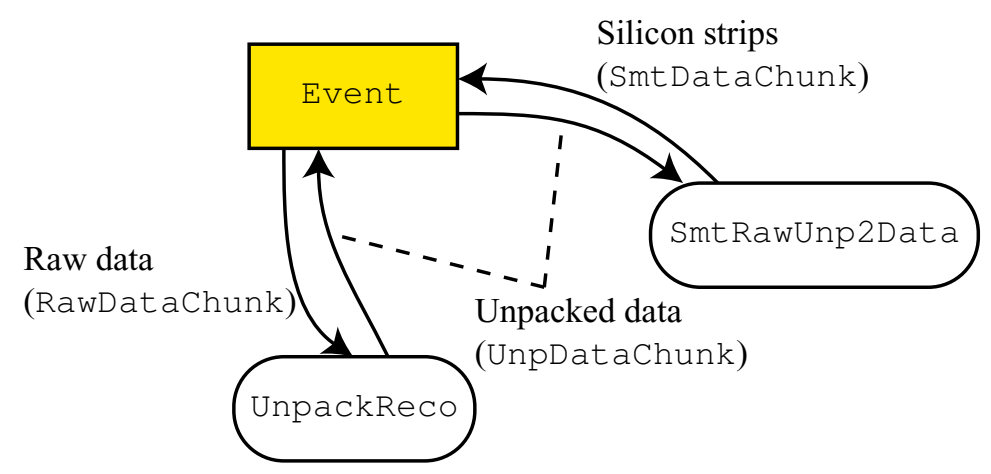

Figure 4.2: Dataflow diagram for the unpacking 
RawDataChunk to UnpDataChunk

The RawDataChunk to UnpDataChunk process is controlled by the D $\varnothing$ framework package PackReco. For each readout module which is listed in the run configuration manager as a VRB belonging to the SMT, PackReco calls the SMT unpacking specific code and passes it the raw data for that VRB.

The unpacking algorithm first reads the fixed size VRB header and then loops over each of the eight HDIs (see figure 4.3). For each active HDI it reads the header and then each SVX data word, continuing for the size given for this HDI in the VRB header. This should then leave the next data word as the end of record marker $(\mathrm{OxCOCO})$. If it does not there is a problem with the data, and this is flagged as an error. Either way, the routine then continues at the beginning of the next HDI (if any).

As each SVX data channel is found it is tagged with a unique identifier formed from the VRB, HDI and chip IDs and the SVX channel number - the front end address - and this and the ADC count are stored in the UnpDataChunk.

An additional data integrity check is to test that the channel numbers within each chip appear only in increasing numerical order. This can catch cases where there are errors in the channel IDs as well as more serious problems, such as when the chip ID is corrupted and is misread as an ordinary data field.

\section{UnpDataChunk to SmtDataChunk}

The DØ framework package SmtRawUnp2Data [57] takes a UnpDataChunk with SMT data and creates a SmtDataChunk. This requires translating the front end address for each channel into the position (in terms of detector elements) of the corresponding strip and applying the appropriate calibration constants. Figure 4.4 gives an overview of the class design for this package (see figure 4.5 for the key to the class diagrams). 


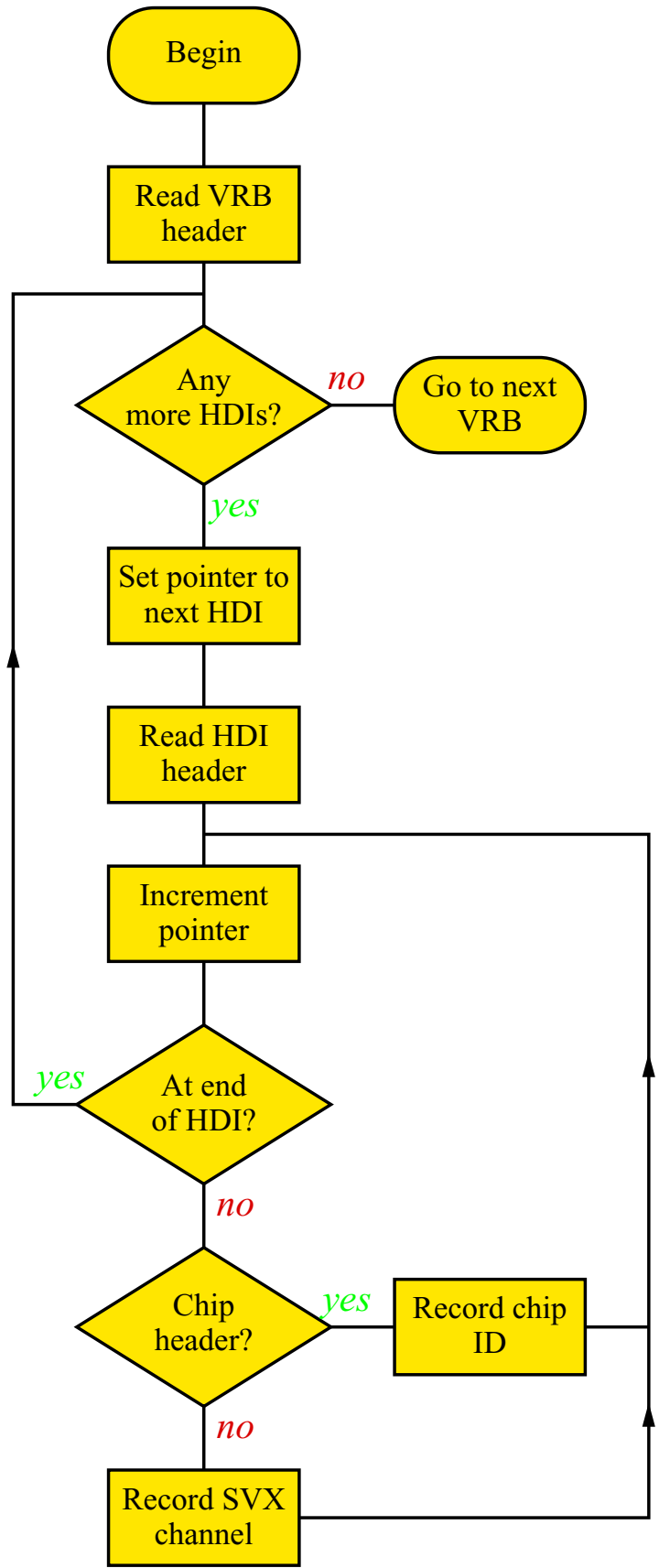

Figure 4.3: Flowchart for the SMT unpacking algorithm 


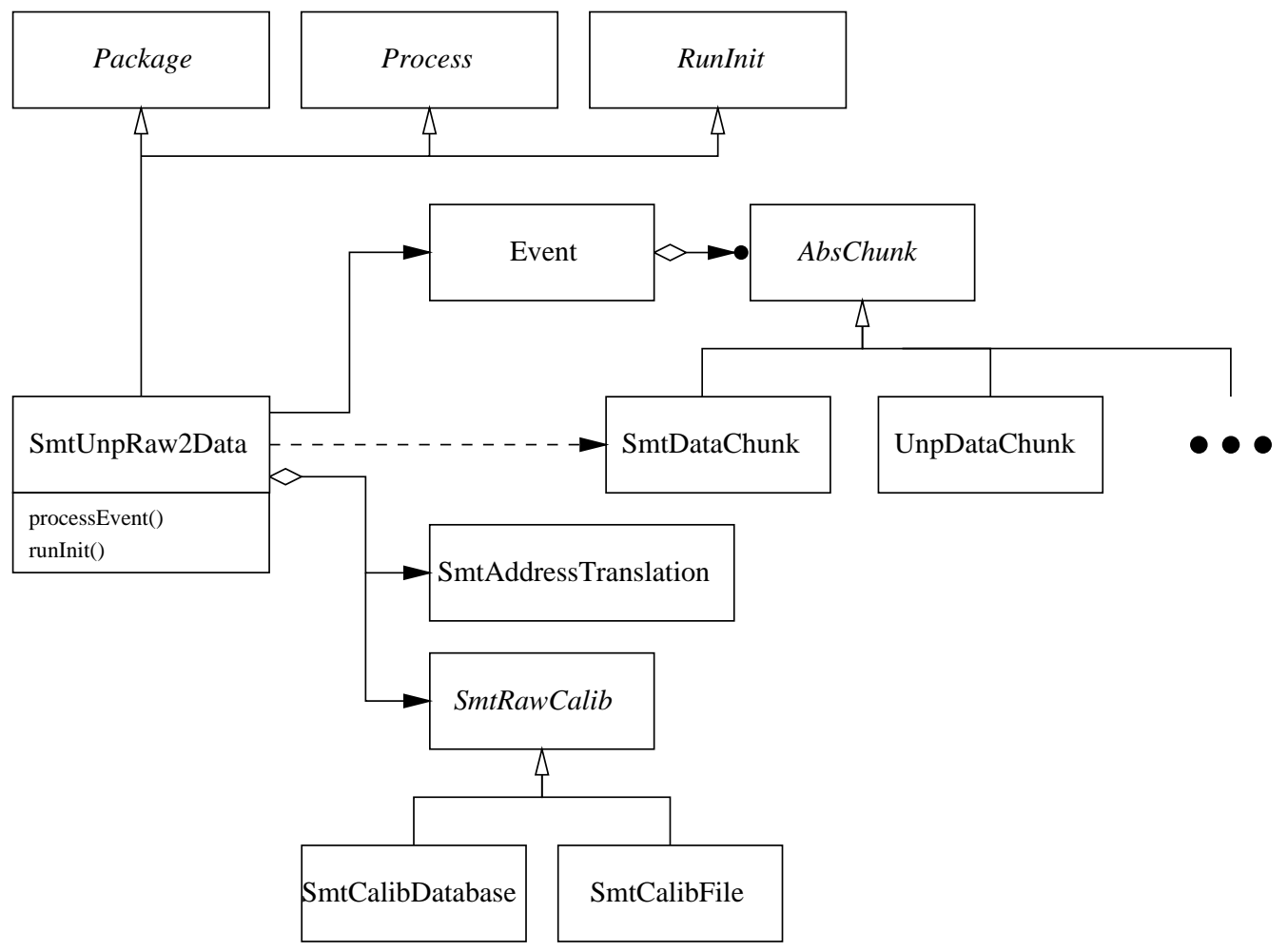

Figure 4.4: The class design of the SmtRawUnp2Data package

Each strip in the detector has a unique ID number - the strip address ${ }^{5}$, and there is a map (determined by the detector cabling) between each electronics channel and each strip. Each channel ID is therefore simply looked up in the map to find the matching strip address.

SmtRawUnp2Data works by looping over each of the SVX channel objects in the UnpDataChunk. It looks up the front end address in the map and if it finds a corresponding strip address it accesses the calibration constants for the strip from the calibration database. It then applies the pedestal ${ }^{6}$ subtraction and multiplies by the gain and adds the strip to the SmtDataChunk. If there is no corresponding strip address in the map then it reports the channel as an error. There is an (adjustable) limit on the number of errors allowed for each HDI, and if this is exceeded the HDI

${ }^{5}$ Formed from either a combination of barrel no/layer no/ladder no/view/strip no or disk type/disk no/wedge no/view/strip

${ }^{6}$ The pedestal is the ADC count for a channel when it contains no signal. 


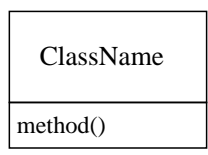

A class and its public interface

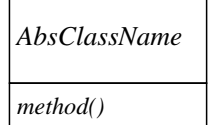

An abstract class (one which defines an interface which is implemented by derived concrete classes)

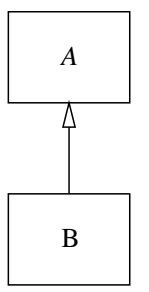

Inheritance: class B inherits from class A

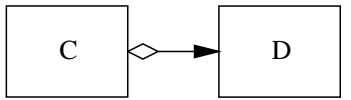

Aggregation (or composition): class D is a member variable of class $\mathrm{C}$

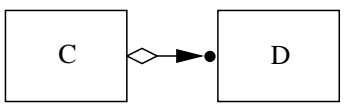

Multiple aggregation: class $\mathrm{C}$ contains many members of class $\mathrm{D}$

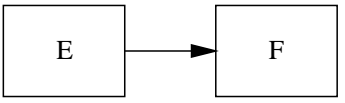

Association (or acquaintance): class E has access to an external instance of class $\mathrm{F}$

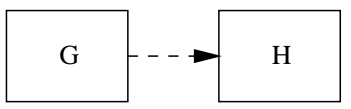

Creation: objects of class $\mathrm{G}$ can create objects of class $\mathrm{H}$

Figure 4.5: Key for class diagrams

is abandoned and processing continues with the channels from the next HDI.

\subsubsection{Level 3 unpacking}

For the Level 3 trigger the required processes to unpack the data are the same, but the trigger code operates under much tighter time constraints. For this reason the Level 3 SMT unpacking code [58] not only merges the two stages described in section 4.3.2 into one, but also combines them with the first stage of the clustering. The purpose is to minimise the number of times the data is copied to and from 


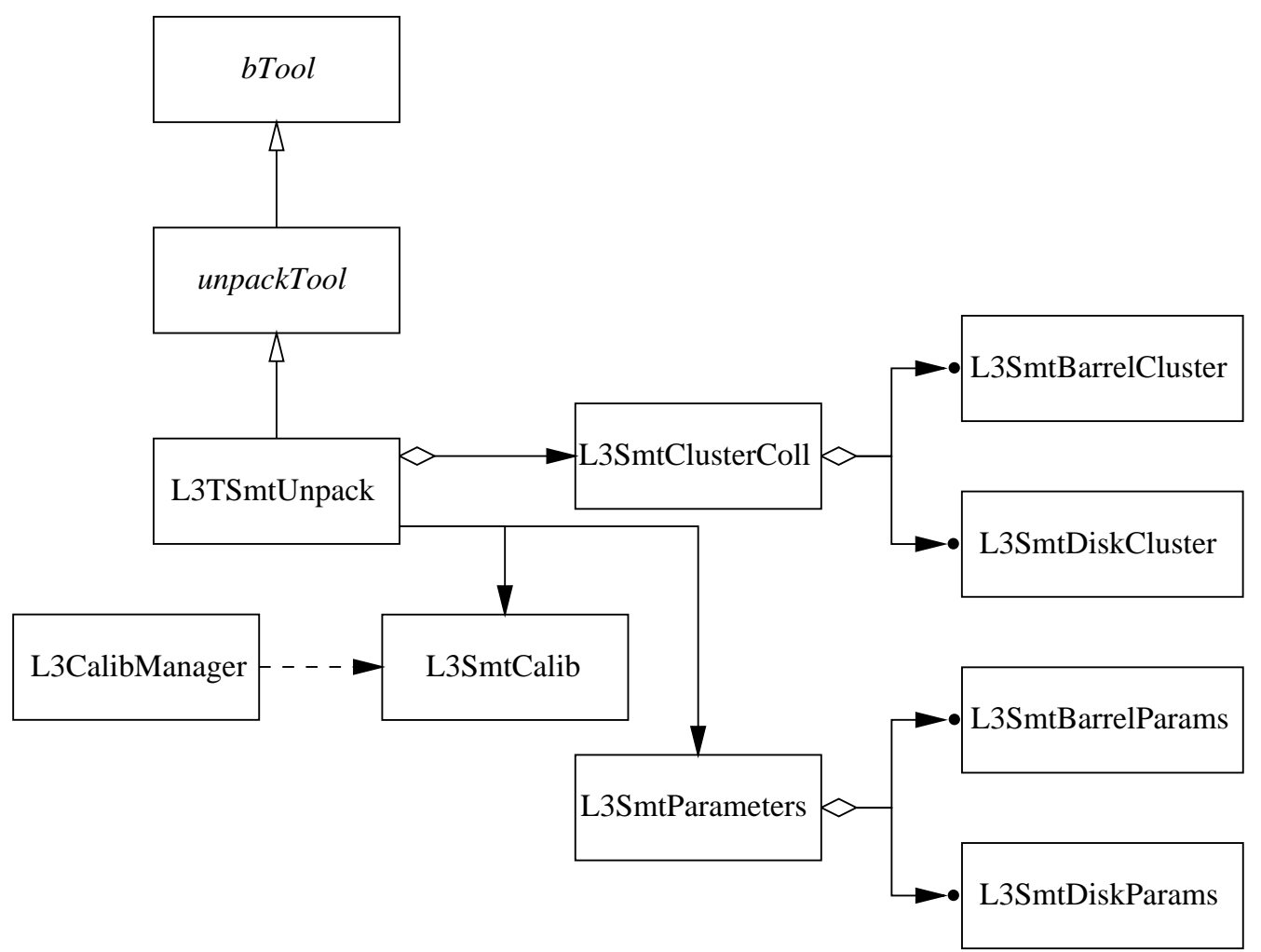

Figure 4.6: The class design of the Level 3 SMT unpacking and clustering tool

memory. The combined unpacking and two dimensional clustering is implemented as a single Level 3 tool (see section 3.5.3). Figure 4.6 shows the class design of the unpacking and clustering tool.

\section{Unpacking}

The method of unpacking is much the same as described before. The difference is that when an SVX channel has been extracted from the data, the strip address translation and the calibration are done immediately and it is then passed directly into the clustering algorithm — clustering is performed 'on the fly' as the strips are unpacked. The clustering is described in section 4.4.

There are a number of extra features also intended to improve the efficiency and speed. Since there are a total of 792,576 channels the calibration information for each individual channel takes up a large amount of memory and requires fetching 


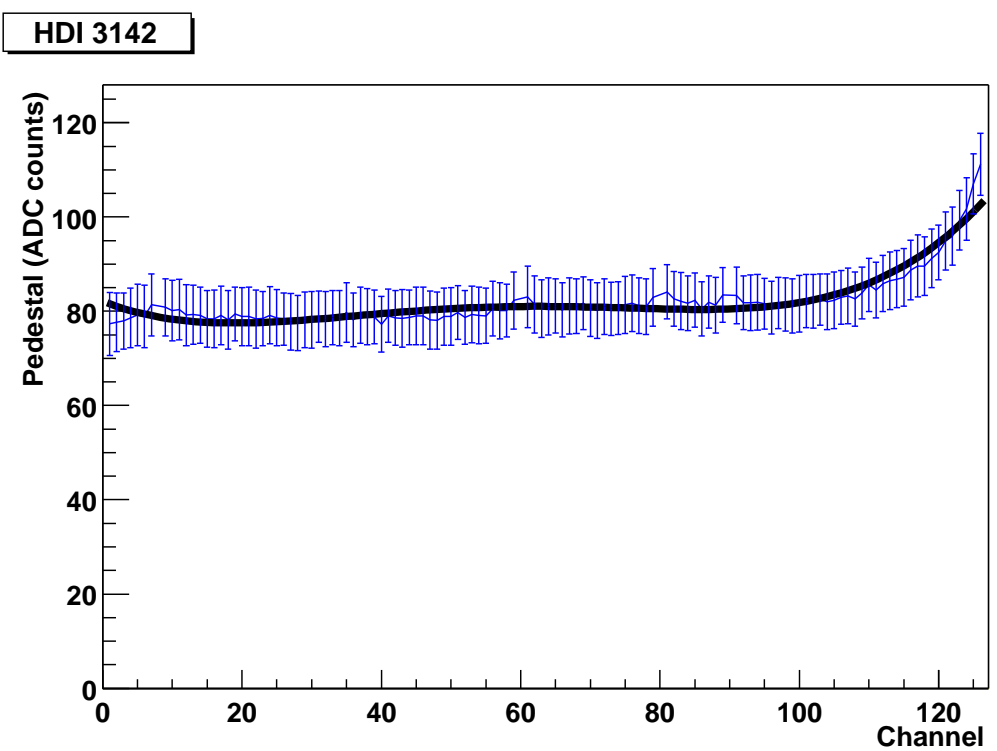

Figure 4.7: Example of pedestals for an SVX chip, with a fitted fourth order polynomial function [59]. The error bars show the standard deviation of the fit used to determine the pedestals, and so show the fluctuations in the pedestal for each channel. The reason for the distribution rising towards the edge of the chip is believed to be due to differing voltage levels across the channels in the chip [60]. Dead channels have been removed from the fit and are responsible for the gaps in the plot.

the calibration constants for each channel from a lookup map, which is a relatively slow process. To reduce the memory usage and the number of map lookups the calibration values are parameterised for each chip. The pedestal values can be approximated by a fourth order polynomial function of the strip number [61]. An example of the pedestals for a single chip are shown in figure 4.7. Parameterising the calibration values like this requires only one lookup for each chip to retrieve the needed values. In addition the front end to strip address translation values can also be stored for each chip in the same map and interpolated to get the address of a particular channel ${ }^{7}$, so only one map lookup is required to fetch all the data required for all the channels belonging to a single chip.

The Level 3 unpacker also supports regional unpacking — it is possible to unpack only a limited region of the detector if requested. The regions supported are re-

\footnotetext{
${ }^{7}$ There is a slight complication that on some detector elements the strip numbers increase in the same direction as the channel numbers, while on others the directions are opposite.
} 
stricted by the organisation of the readout and the fact that the smallest complete unit which it is sensible to unpack is a complete VRB. The most obvious (and currently available) regions are the six $60^{\circ}$ barrel sectors, the $\mathrm{F}$ disks, and the $\mathrm{H}$ disks.

\subsubsection{Packing}

Running the Level 3 SMT unpacker, and consequently the Level 3 trigger simulator, requires raw data. It is therefore necessary to be able to create simulated raw data from Monte Carlo. This process is basically the reverse of the offline unpacking procedure. There is a framework package SmtData2RawUnp which creates a UnpDataChunk from a SmtDataChunk by undoing the calibration and translating the strip addresses into front end addresses. The class design for SmtData2RawUnp is shown in figure 4.8. The framework package PackReco then calls the specific packing routines for each of the subdetectors and writes out a RawDataChunk with the combined raw data.

\subsection{Clustering in the Level 3 trigger}

Clustering is done as a two stage process in the Level 3 trigger. The first stage is performed simultaneously with the unpacking and is to group adjacent hit strips into 'two dimensional' clusters. The axial and the $90^{\circ}$ barrel ladders can directly provide $r \phi$ and $z$ positions respectively. Since the $2^{\circ}$ barrel and the disk strips are not aligned along the major axes of the detector these clusters are not directly useful on their own, but only in combination with the clusters from the other side of the ladder or wedge. This combining of the two dimensional clusters into three dimensional clusters is carried out by a different Level 3 tool from the unpacking and is described in [62].

Converting the strips into a cluster with a given position requires knowlege of the 


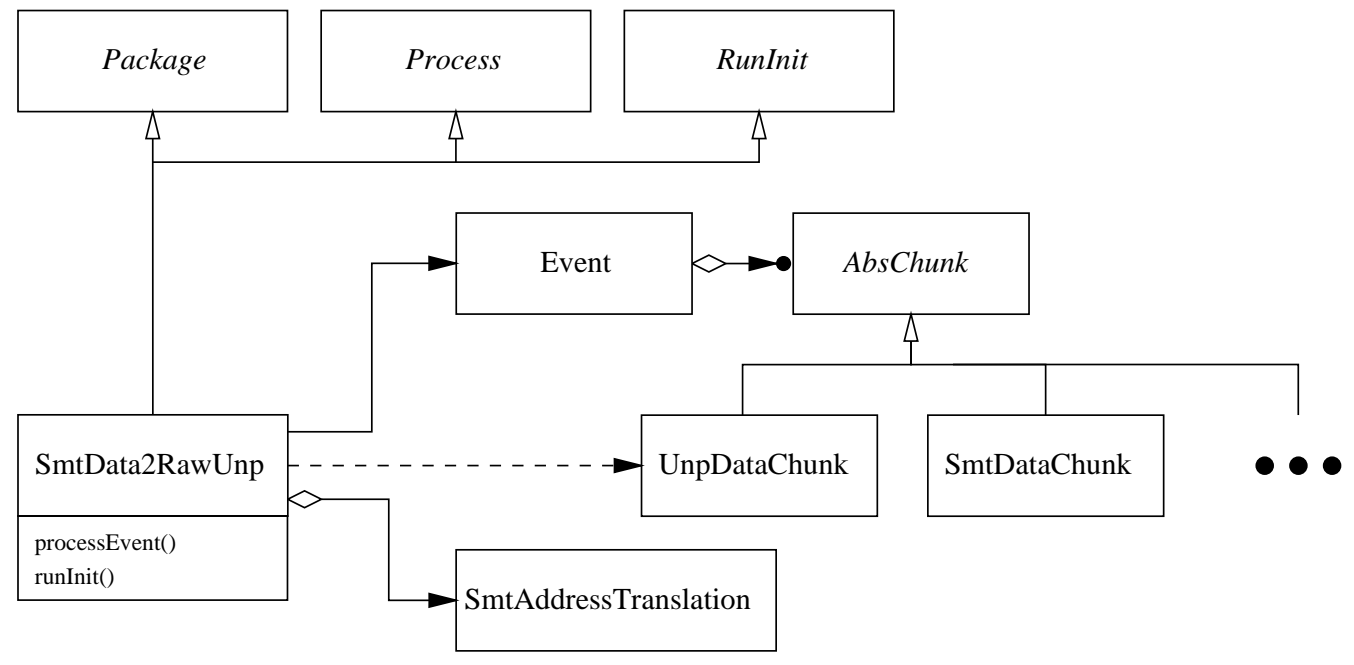

Figure 4.8: The class design of the SmtData2RawUnp package

coordinate systems used in the silicon detector. These are described in the next section.

\subsubsection{SMT Coordinate systems}

\section{Local coordinates}

Each ladder or wedge has its own local coordinate system [63, 64]. The local z axis is directed towards the SVX chips, the local y axis is directed from the $p$ side of the detector to the $n$ side (or on an $\mathrm{H}$ disk, from the first side to the second), and the $x$ axis is oriented to form a right handed coordinate system. The origin of the local coordinates is positioned at the centre of the ladder or wedge. Clusters are formed by grouping together all the strips which collected charge from the same particle passing the silicon and reducing it to a single position measurement. This one dimensional position - the cluster centroid - is measured along a line perpendicular to the orientation of the strips with zero again at the centre of the ladder/wedge.

On a double sided detector there is a different stereo angle on each side. Given a centroid position on each side then the point where they cross (figure 4.10) is given 


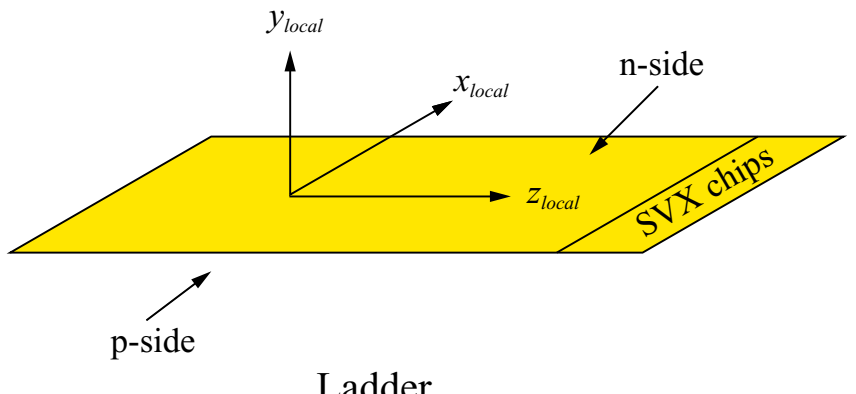

Ladder

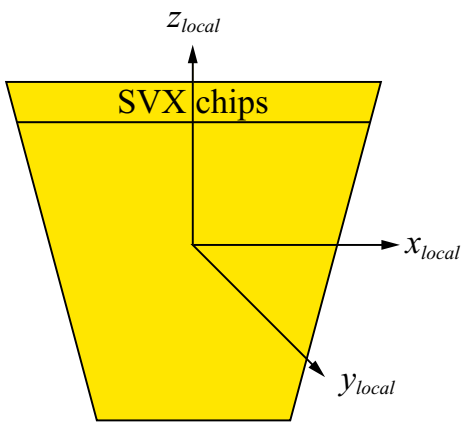

Wedge

Figure 4.9: The local coordinate system for ladders and wedges.

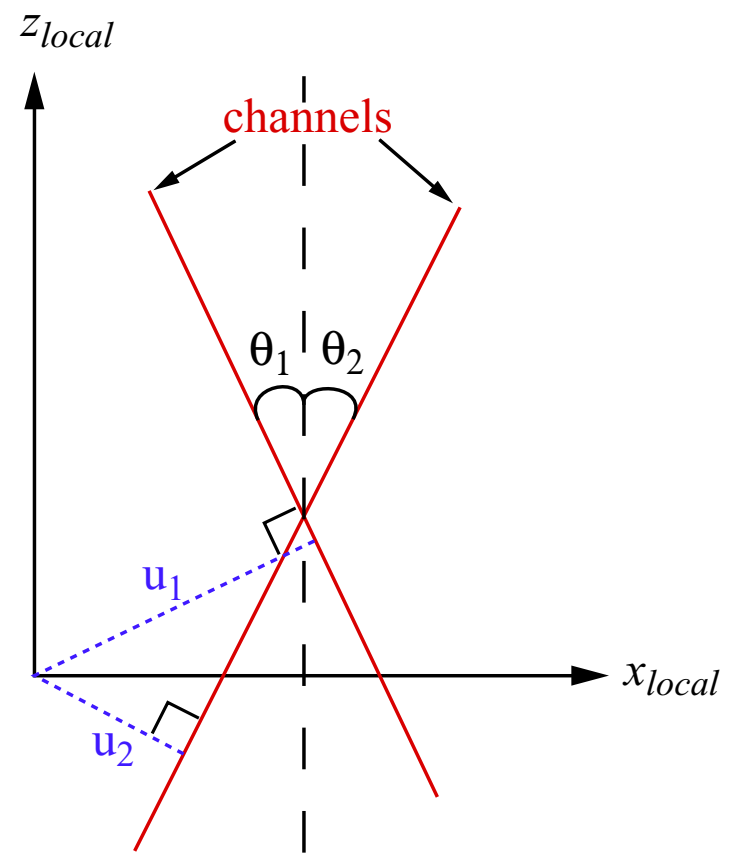

Figure 4.10: Calculation of hit position 
by

$$
\begin{aligned}
x_{L} & =\frac{u_{1} \sin \theta_{2}-u_{2} \sin \theta_{1}}{\cos \theta_{1} \sin \theta_{2}-\sin \theta_{1} \cos \theta_{2}} \\
z_{L} & =\frac{u_{1} \cos \theta_{2}-u_{2} \cos \theta_{1}}{\cos \theta_{1} \sin \theta_{2}-\sin \theta_{1} \cos \theta_{2}}
\end{aligned}
$$

where $u_{1}, u_{2}$ are the centroid positions and $\theta_{1}, \theta_{2}$ are the stereo angles on sides 1 and 2 respectively. On barrel ladders $\theta_{1}=0$ and this can be simplified:

$$
\begin{aligned}
& x_{L}=u_{1} \\
& z_{L}=\frac{u_{1} \cos \theta_{2}-u_{2}}{\sin \theta_{2}}
\end{aligned}
$$

On $90^{\circ}$ stereo ladders it becomes even simpler

$$
\begin{aligned}
& x_{L}=u_{1} \\
& z_{L}=-u_{2}
\end{aligned}
$$

This implies that $u_{2}$ is measured in the opposite direction to the local $\mathrm{z}$ axis.

\section{Global coordinates}

The global coordinate system is common to all subdetectors in DØ. Figure 4.11 shows how the global coordinates are defined relative to the SMT detector. The Level 3 software needs to be able to transform local coordinates to the global system quickly. It does not use the full geometry system but makes the simplifying assumption that the ladders and wedges are all flat [65]. This makes the transformation a simple linear one

$$
\left(\begin{array}{l}
x_{G} \\
y_{G} \\
z_{G}
\end{array}\right)=\mathbf{r}_{0}+x_{L} \mathbf{r}_{1}+y_{L} \mathbf{r}_{2}
$$


where $\mathbf{r}_{0}$ is the vector from the global origin to the centre of the ladder/wedge, $\mathbf{r}_{1}$ is the global coordinate vector corresponding to the vector $(1,0,0)$ in the local ladder/wedge coordinates, and similarly $\mathbf{r}_{2}$ corresponds to $(0,0,1)$.

\subsubsection{Two dimensional clustering}

The input to the clustering algorithm is the calibrated energy for each read out strip produced by the unpacker (see section 4.3.3). The clustering is done 'on the fly': each strip is sent directly from unpacking to clustering without any intermediate storage.

The basic algorithm for forming the clusters is as follows: as each new strip that has an energy above a set threshold value is added, its position address is checked to see if it is positioned on the same detector element and view as the current cluster, and to see if there is a gap of no more than one strip between it and the previous strip in the cluster. If this is the case then the strip belongs to the current cluster. If this is not the case or this is the first strip unpacked then it marks the start of a new cluster, so the geometry calculations for the existing cluster (if any) are done and it is stored in the list of clusters. The geometry calculations and storage of the final cluster must be explicitly forced as an extra step when all the strips have been read out as there is nothing else to tell it that the last cluster is finished.

The readout of the stereo side of the $90^{\circ}$ detectors is multiplexed - each SVX channel is connected to two strips which are separated by approximately $6 \mathrm{~cm}$ along the z-axis. In this case there is no way to know which half of the ladder the hit belongs to, so each of the stereo clusters on these ladders is duplicated, with the copy offset by $6 \mathrm{~cm}$.

The position of the cluster is given by the pulse height weighted average of the strips:

$$
\bar{n}=\frac{\sum n_{i} w_{i}}{\sum w_{i}}
$$




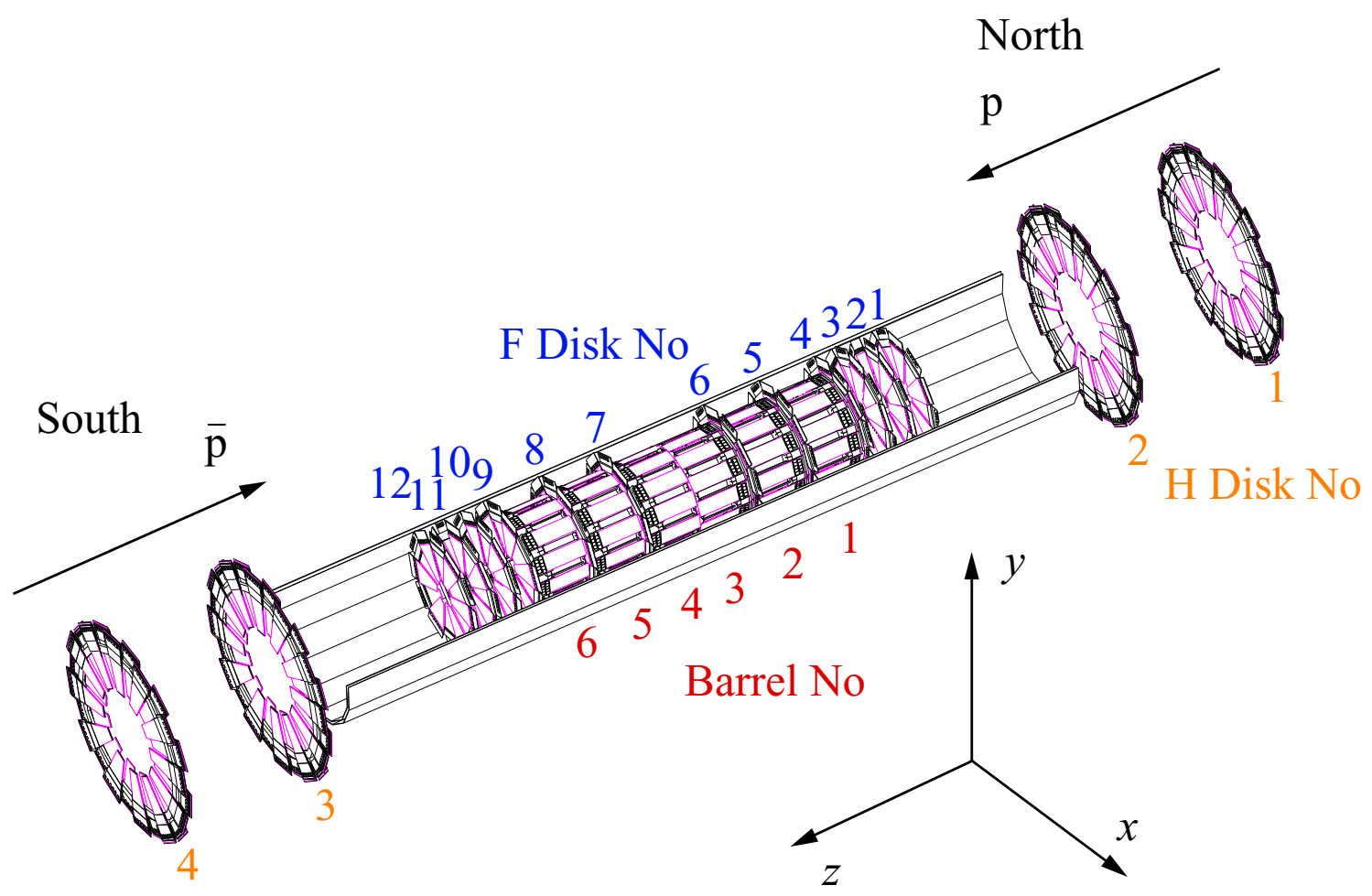

Figure 4.11: The global coordinate system. The proton beam is along the positive z-axis.

where $n_{i}$ is the strip number of the $i$ th strip and $w_{i}$ is the deposited energy in that strip. The totals are easily accumulated as the strips are added to each cluster. The mean strip number now needs to be converted first into the local coordinates of the ladder or wedge, and then into the global coordinate system. The first stage is to calculate the centroid position, which is given by

$$
u=u_{1}+(\bar{n}-1) p
$$

where $u_{1}$ is the position of the centre of the first strip and $p$ is the pitch of the strips. (The -1 is needed because the strip numbering starts at 1.) Converting the centroid position into the local coordinates depends on the detector element. For 
axial barrel clusters

$$
\begin{aligned}
& x_{L}=u \\
& z_{L}=0
\end{aligned}
$$

For $90^{\circ}$ barrel clusters

$$
\begin{aligned}
& x_{L}=0 \\
& z_{L}=-u
\end{aligned}
$$

For $2^{\circ}$ barrel and disk detectors the $2 \mathrm{~d}$ clusters are not much use except when combined with other clusters. For this reason the coordinate values are not particularly important (as opposed to the centroid value), but they are set to

$$
\begin{aligned}
& x_{L}=u \cos \theta_{2} \\
& z_{L}=0
\end{aligned}
$$

Finally, these local coordinates are converted to the global coordinates by using equation (4.4).

The performance of the clustering is determined by matching the reconstructed clusters to the hits in the GEANT simulation of the detector. The method is described in $[62]$.

The residuals for clusters on the axial side of barrel ladders is shown in figure 4.12. The position resolution is $\sim 7 \mu \mathrm{m}$. The efficiency and misidentification rate for these clusters is shown in figure 4.13. The fall in efficiency on the lower levels is due to overlapping hits, which are not separated by the cluster algorithm used [66]. The test beam data for the silicon obtained resolutions of $9 \mu \mathrm{m}$ for $50 \mu \mathrm{m}$ pitch detectors $[39,67]$.

The $z$ resolution of the $90^{\circ}$ stereo barrel ladders is shown in figure 4.14. The resolution on these ladders is $\sim 23 \mu \mathrm{m}$. 


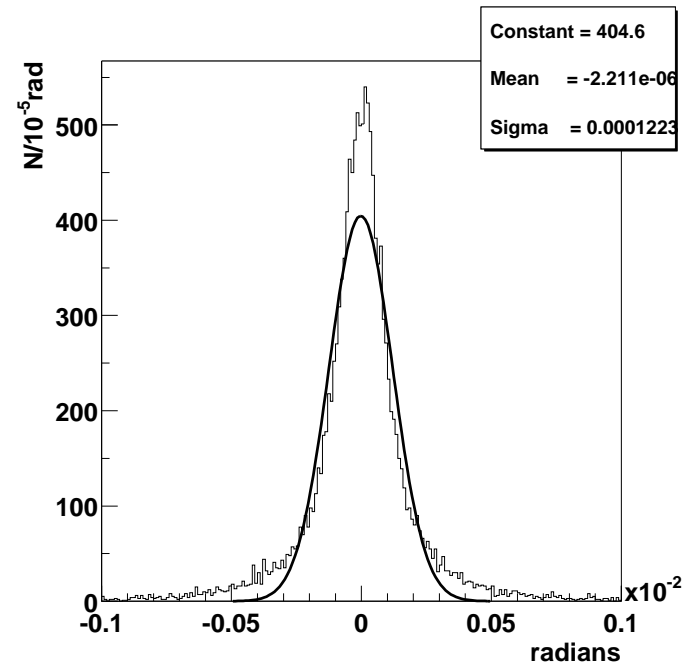

(a) Difference in $\phi$ between cluster and Monte Carlo

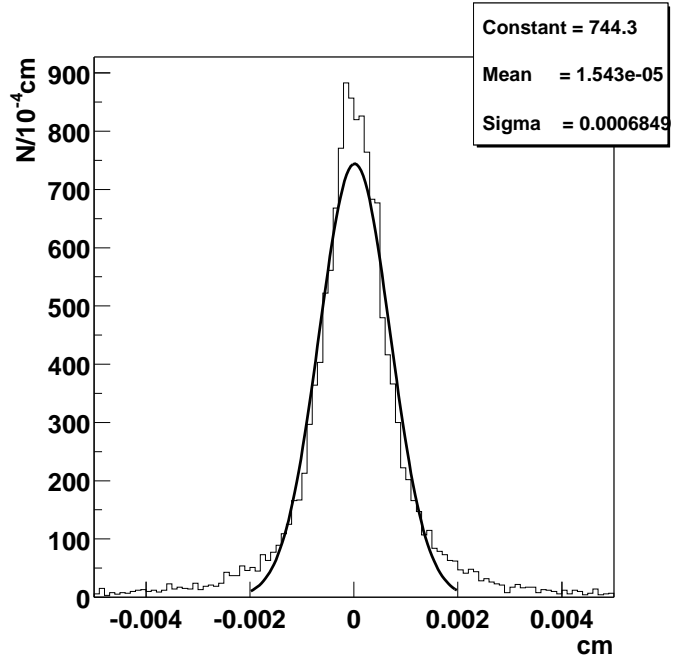

(b) Resolution in ladder local $x$ coordinate

Figure 4.12: Monte Carlo spatial resolution of the axial barrel clusters

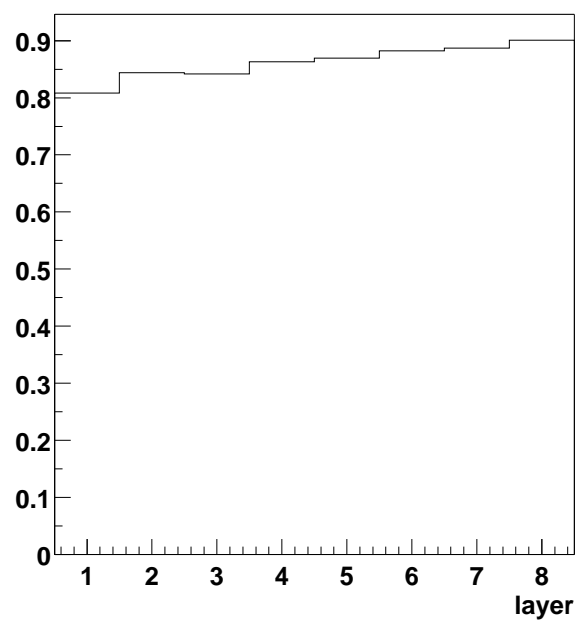

(a) Efficiency

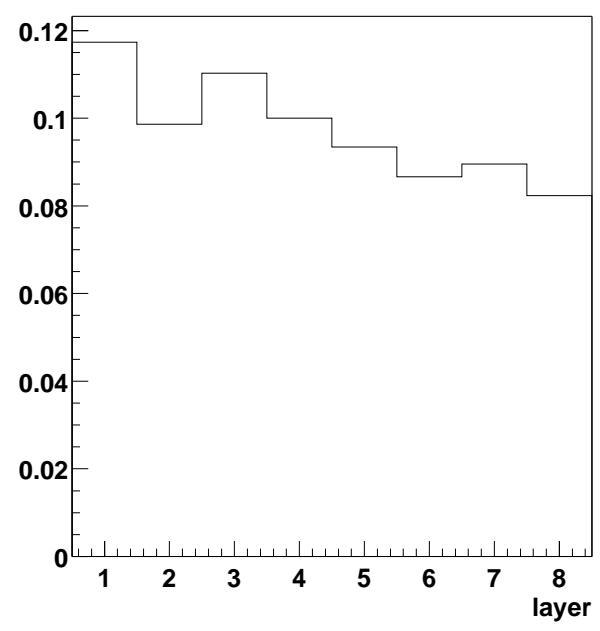

(b) Misidentification

Figure 4.13: Monte Carlo efficiency and misidentification of axial clusters. Efficiency is defined as (number of matched clusters)/(total number of MC hits), and misidentification is defined as (number of unmatched clusters)/(total number of MC hits) 


\subsubsection{Three dimensional clustering}

The method to create the three dimensional clusters are described in [62]. Since the only way to measure the performance of the $2^{\circ}$ barrel clusters and any of the disk clusters is to use the three dimensional cluster positions, some of the clustering results are given here.

Figure 4.17 shows the spatial resolution of the $z$ position of the three dimensional clusters on $2^{\circ}$ ladders. The resolution achieved is $\sim 260 \mu \mathrm{m}$. 


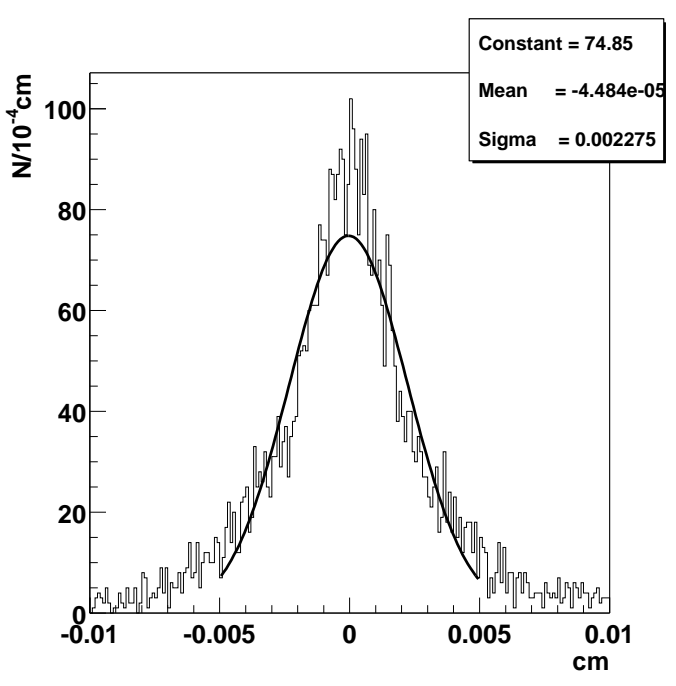

Figure 4.14: Monte Carlo spatial resolution in $z$ of $90^{\circ}$ stereo barrel clusters

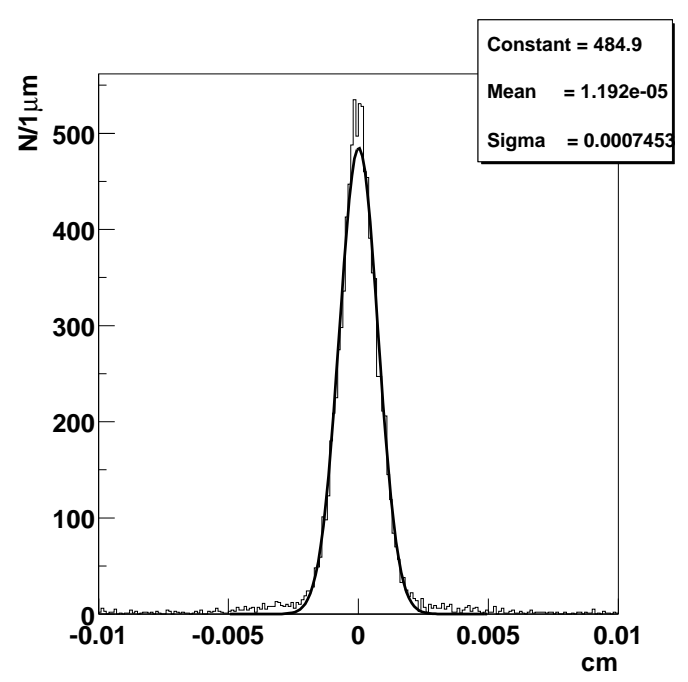

(a) Resolution in $x_{\text {local }}$

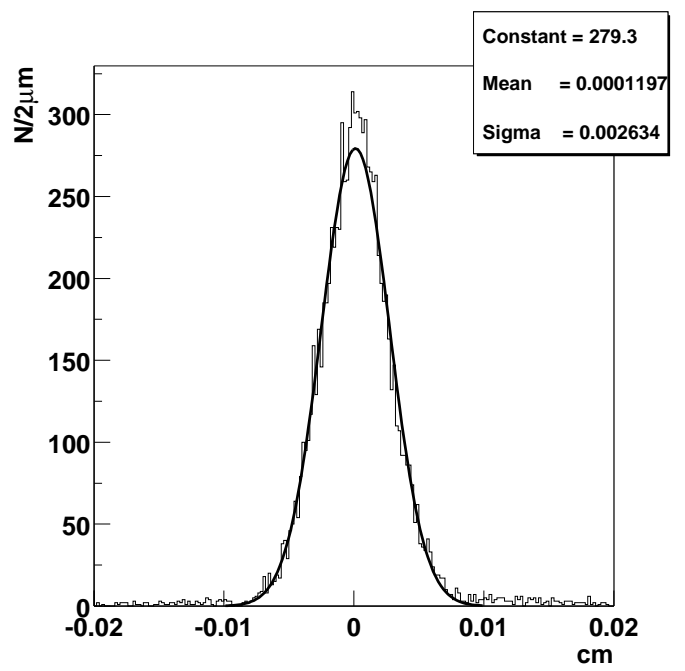

(b) Resolution in $z_{\text {local }}$

Figure 4.15: Monte Carlo spatial resolution of F disks 


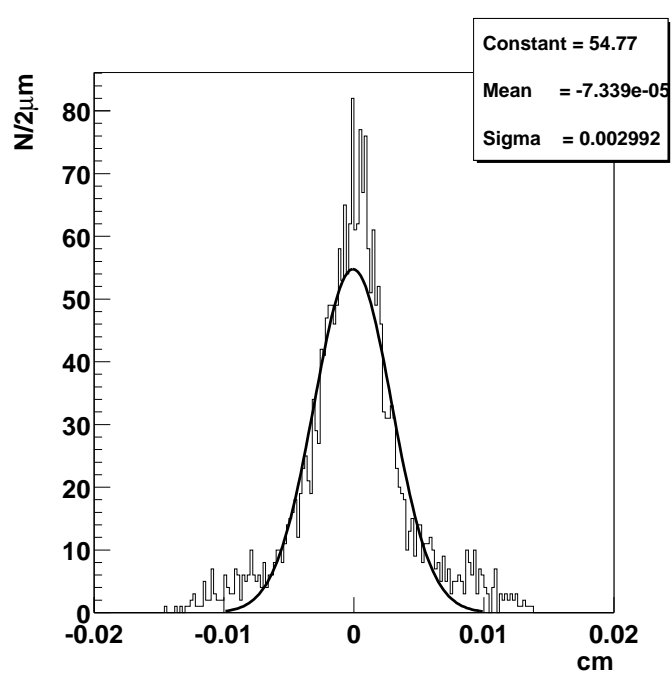

(a) Resolution in $x_{\text {local }}$

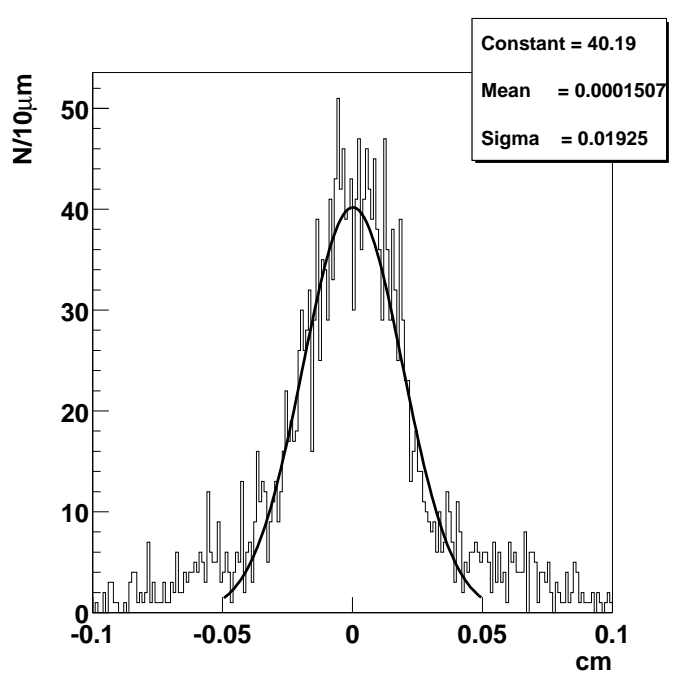

(b) Resolution in $z_{\text {local }}$

Figure 4.16: Monte Carlo spatial resolution of $\mathrm{H}$ disks

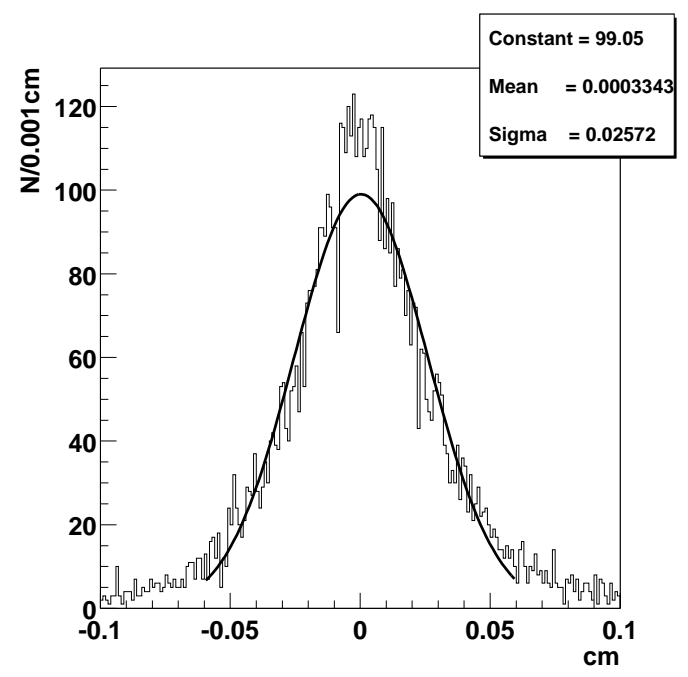

Figure 4.17: Monte Carlo spatial resolution in $z$ of $2^{\circ}$ stereo barrel clusters 


\subsection{Results from data}

\subsubsection{Results from the cosmic ray test}

Before the SMT was installed in the detector, it was tested using cosmic rays. The data collected was reprocessed offline using the Level 3 software. The normal Level 3 unpacking and clustering was used, and then tracks were searched for using a specialised straight line track finder [68]. Figure 4.18 shows a track found in this data. The results obtained were in good agreement, with those from the same events processed by the offline track reconstruction code (figure 4.19).

\subsubsection{Results from first collisions}

The first collisions in Run II of the Tevatron occurred on 3 April 2001. Only a small portion of the SMT was fully connected at that time, and the initial data was used to adjust the timing of the various stages in the readout. The Level 3 unpacking and clustering was used to process a slightly later run (run 117125, taken on 6 April), and the output was searched by eye for possible tracks. The central magnetic field was not switched on while this data was taken, so tracks would appear as straight lines. Figure 4.20 shows an event found very early on. The cosmic ray track finder referred to in section 4.5.1 was modified to cope with horizontal tracks and run on this event, producing the fitted line shown. The track in this event was also independently found and fitted using the offline SMT software and tracking code [69]. 


\section{Event 16}

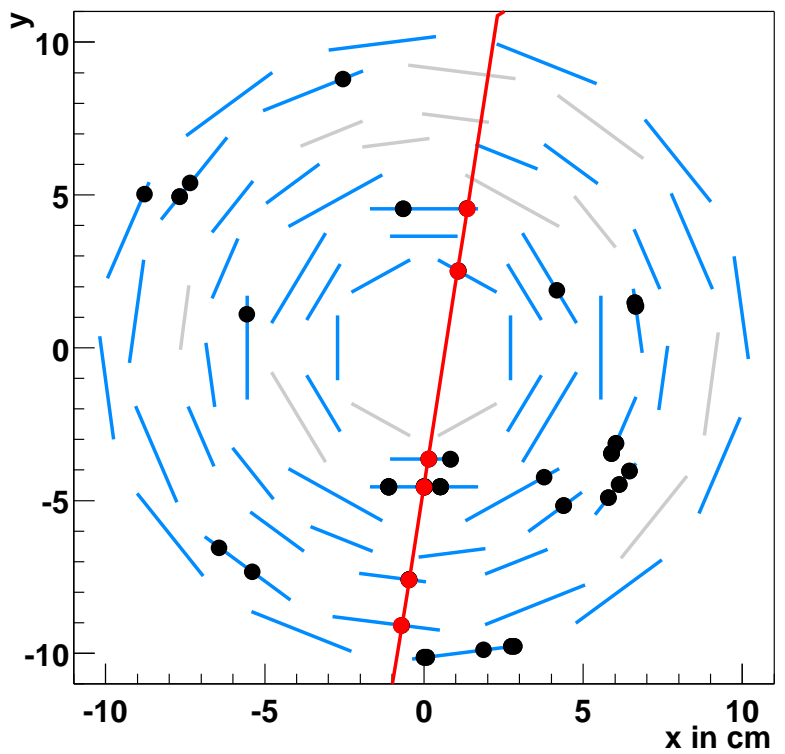

(a) $x y$ view

\section{Event 16}

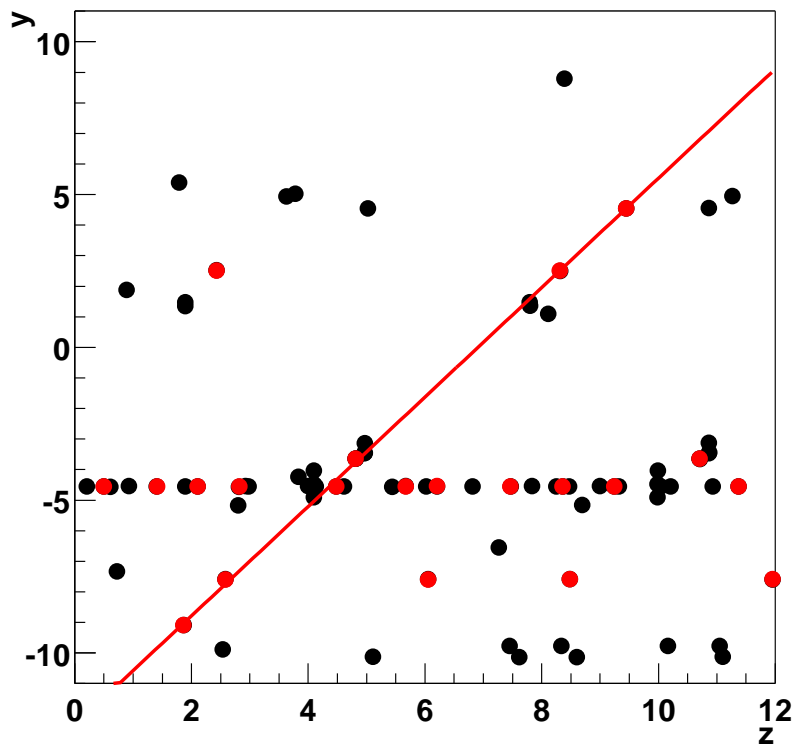

(b) $y z$ view

Figure 4.18: A track from the cosmic ray test [68]. In the $y z$ view all the hits which can be associated with one of the axial clusters used to form the track in the $x y$ view are shown coloured differently. Due to ghost clusters and the multiplexing on the $90^{\circ}$ stereo ladders there can be a large number of potential stereo hits for each axial cluster. 


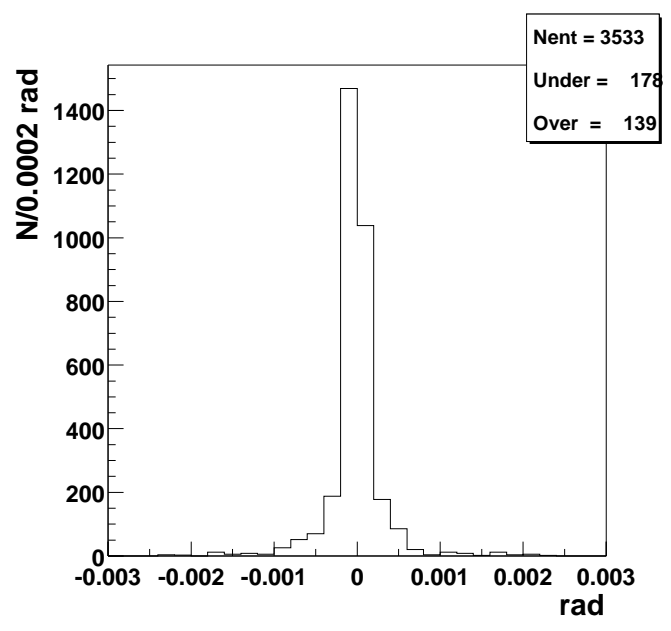

(a) Difference between Level 3 and offline axial cluster $\phi$ coordinates

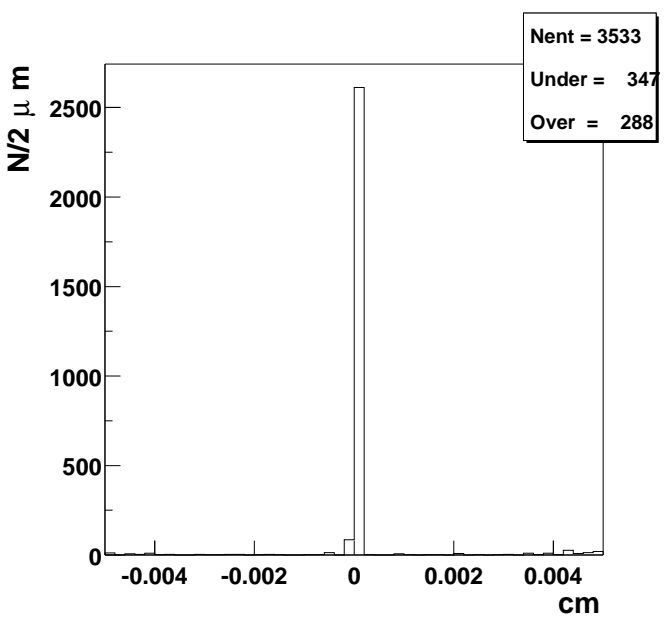

(b) Difference between Level 3 and offline $90^{\circ}$ stereo $z$ positions

Figure 4.19: Comparison of Level 3 and offline cosmic ray hit positions [68]. The main source of differences between the two is that the offline reconstruction used a more sophisticated (event-byevent) pedestal subtraction method than the Level 3 code. This caused a increased rate of fake clusters due to an higher level of unspressed noise in the Level 3 unpacker.

\subsection{Timing results for Level 3 unpacking and clus- tering}

The Level 3 trigger algorithms are required to run quickly - the average time allowed to process each event is $\sim 100 \mathrm{~ms}$. Figure 4.21 shows the results of running the SMT unpacking and clustering tool over Monte Carlo samples of different event types. The computer used for these tests was a Linux PC with the same processor speed as that of the actual Level 3 filter nodes (1 GHz Intel Pentium III).

The tool must also behave reasonably in the presence of minimum bias events. Figure 4.22 shows the effect of adding minimum bias events to $Z \rightarrow \mu^{+} \mu^{-}$events. Unsuprisingly, the running time scales linearly with the number of minimum bias events.

Another potential problem which affects the timing is the amount of noise in the detector. The Monte Carlo samples have some simulated noise (correlated and 

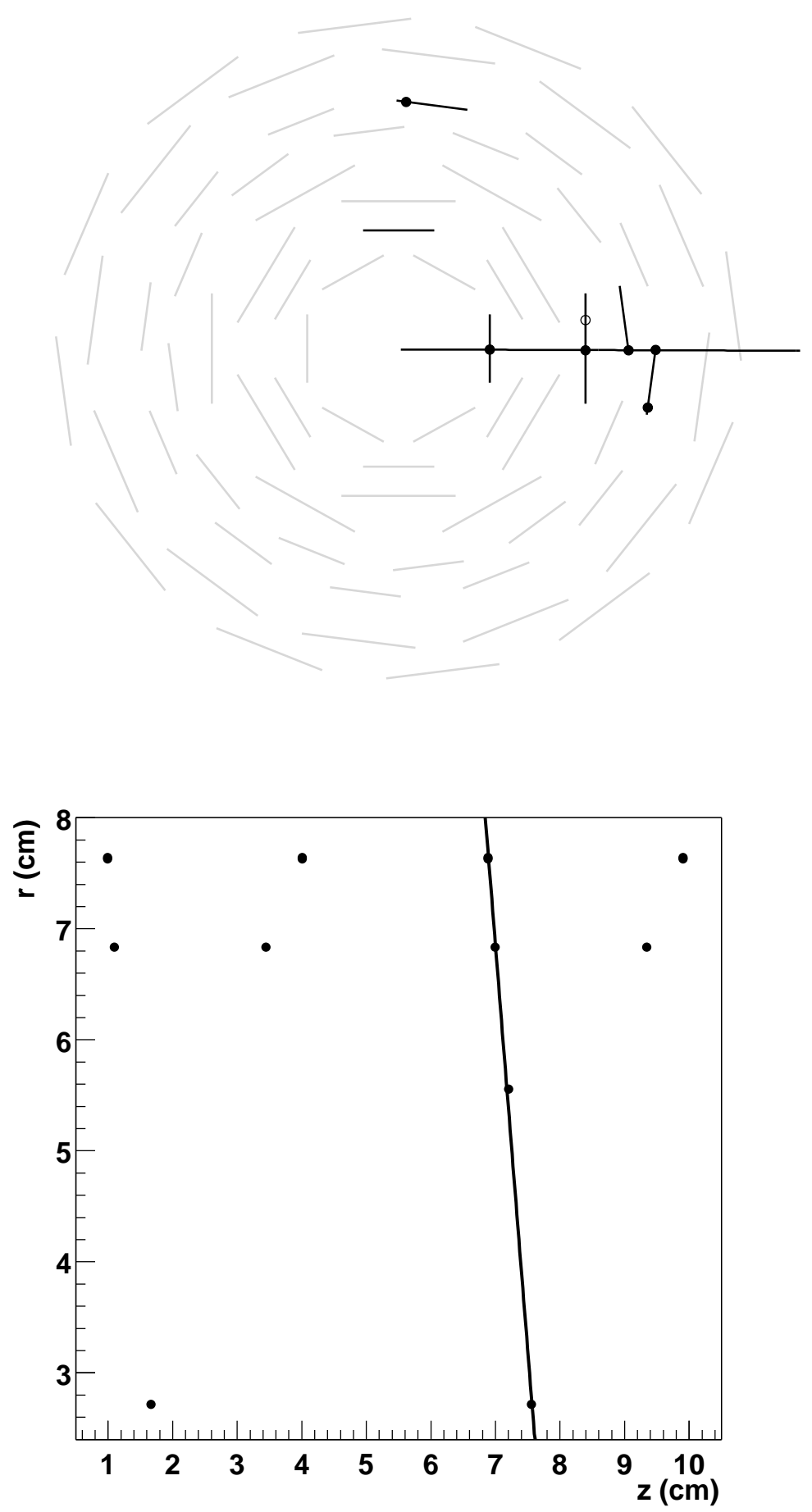

Figure 4.20: The first track from $p \bar{p}$ collisions found in the SMT. In the $x y$ view, the solid circles are hits with where an axial cluster has been matched to a stereo cluster, and the open circle is a axial cluster with no matching stereo cluster. Only the ladders shown as black lines were read out. In the $r z$ view, only hits with matching axial and stereo clusters are shown. 
random noise around any fired strip and random noise added to all channels, and then a channel ADC threshold cut), but figure 4.23 shows the effect of forcing a random fraction of the total channels to have a large ADC count. These extra channels cause a large number of additional clusters to be formed (1\% corresponds to $\sim 3900$ addtional clusters, while the signal data only produces $\sim 300-1200$ ). This shows that it is important to remove known noisy strips from the data, not just to reduce the number of fake clusters, but also to reduce the running time.

Figure 4.24 shows the tool running time on a sample of real data. The detectors and readout were still being commissioned at this point and some of the ladders were producing incorrect output (for example, occassional missing HDI end of record markers, and invalid chip IDs), so some extra time is required to write information on this to the error log. However, the overally time taken is not seriously in excess of the Monte Carlo unpacking time.

These results show that the SMT unpacking and clustering time is reasonable within the total Level 3 filtering time budget. 

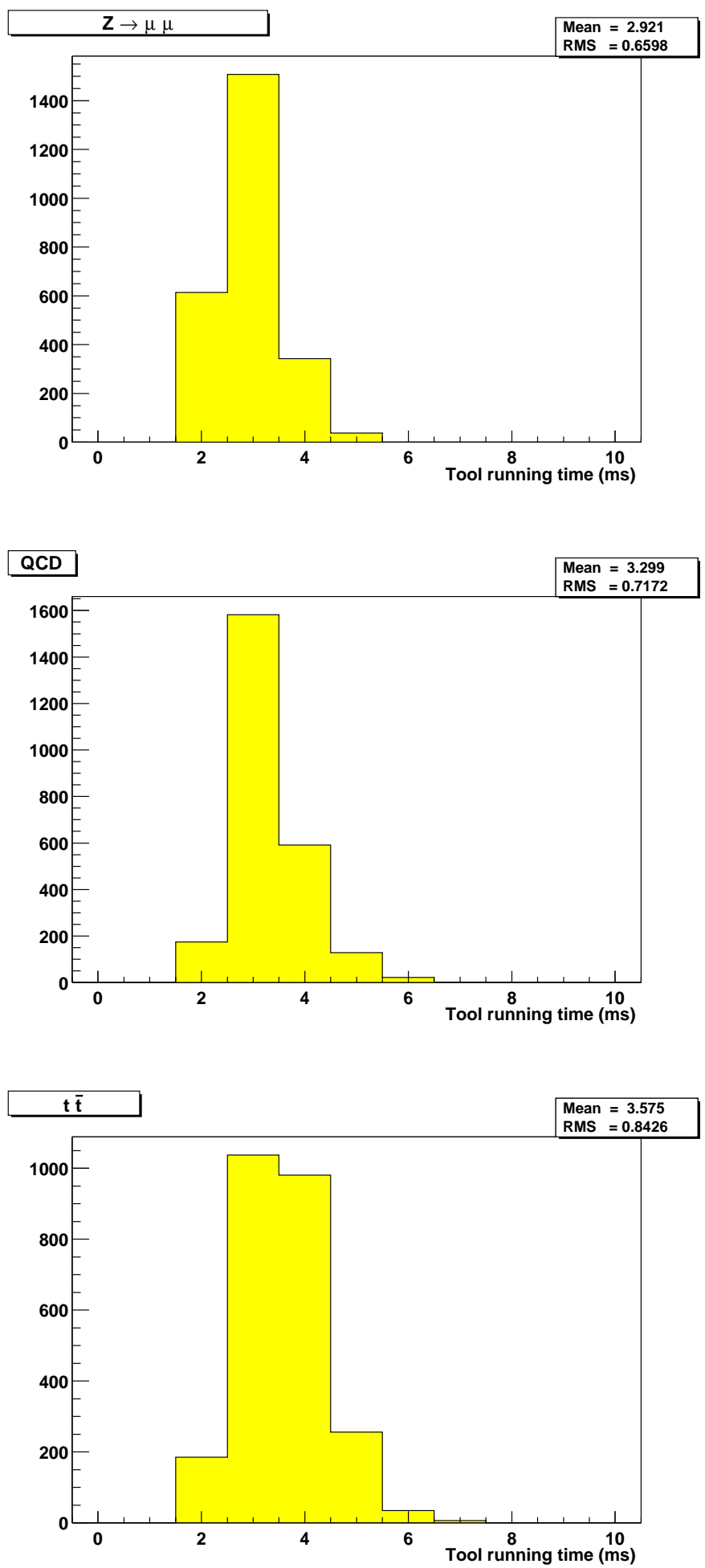

Figure 4.21: SMT unpacking and clustering time (barrels only) for different Monte Carlo event types. In all cases no additional minumum bias interactions were present. 


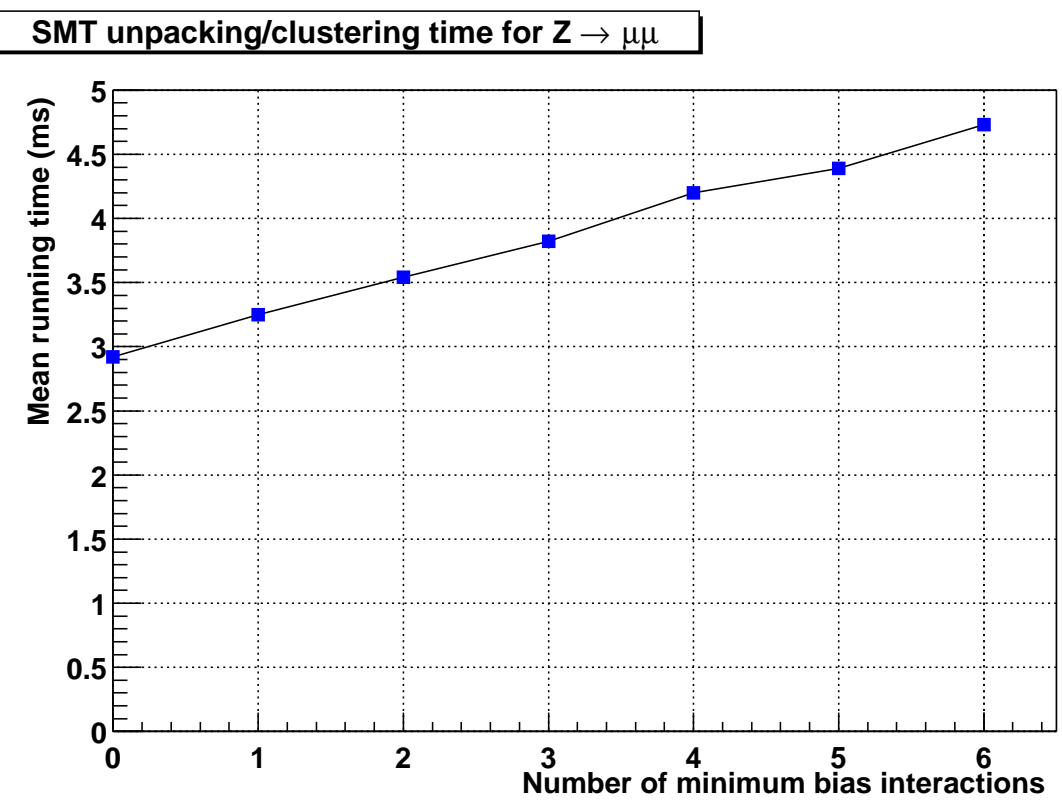

Figure 4.22: The unpacking and clustering time (barrels only) for $Z \rightarrow \mu \mu$ events with varying numbers of added minimum bias interactions.

\section{SMT unpacking/clustering time}

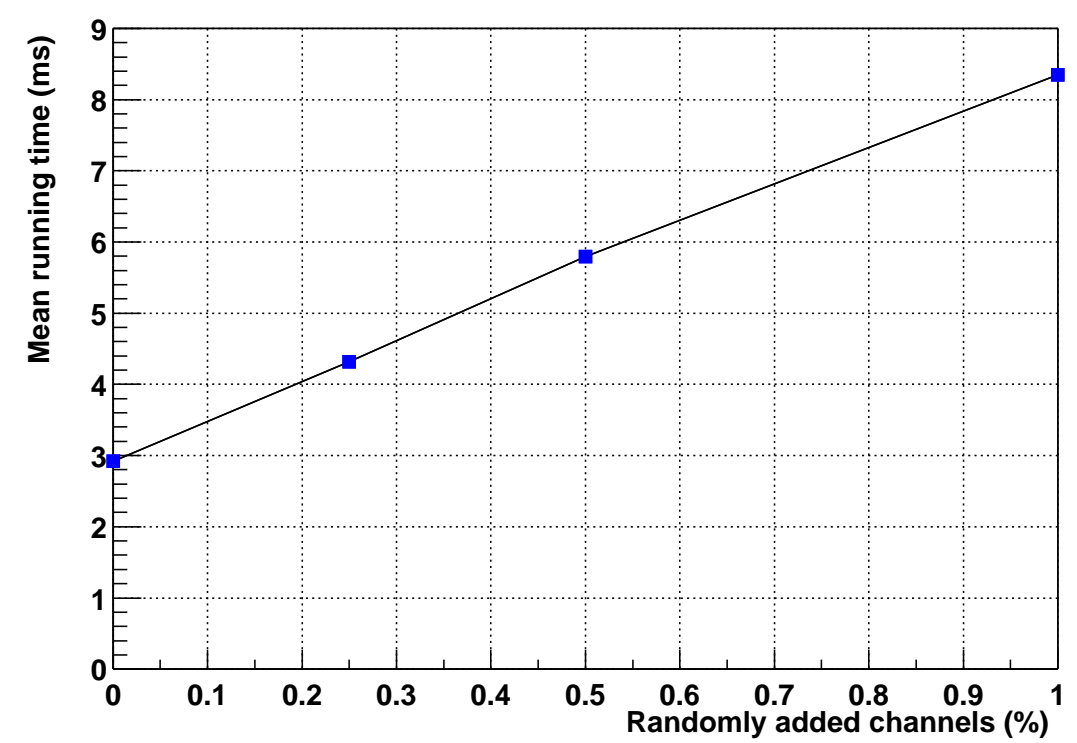

Figure 4.23: The unpacking and clustering time (barrels only) for $Z \rightarrow \mu \mu$ events with varying added noise level 


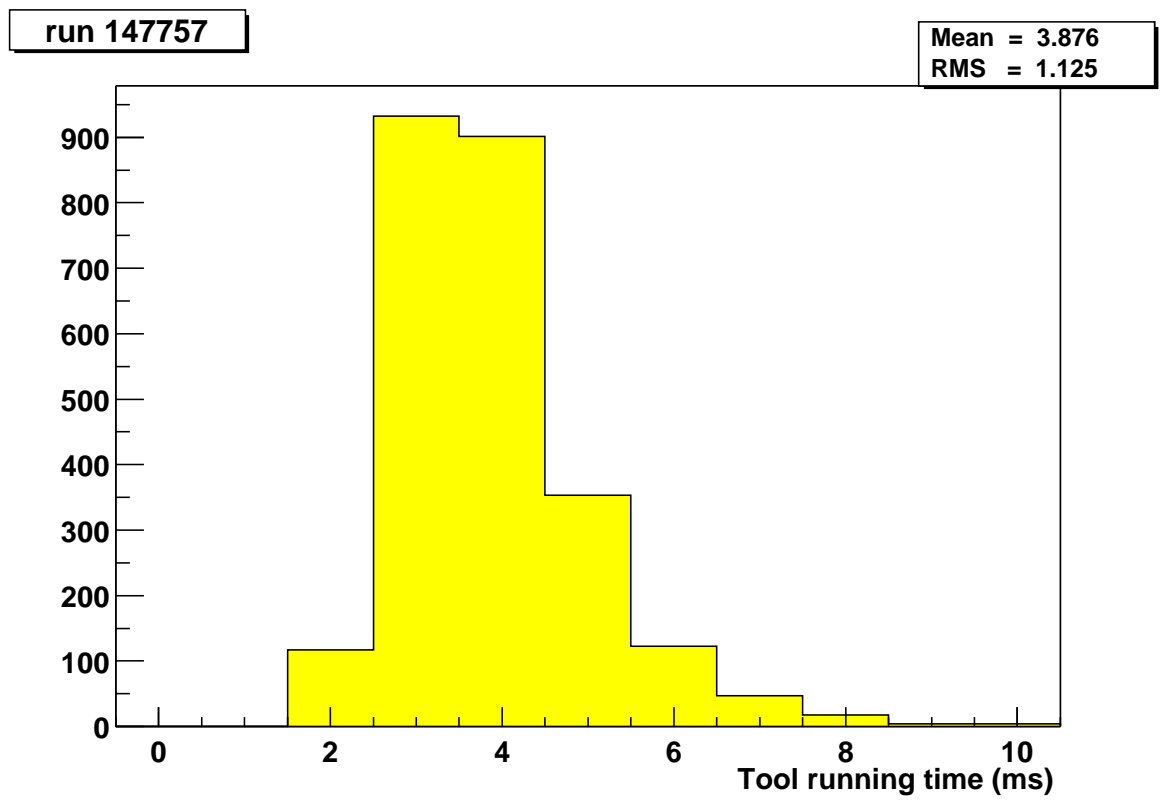

Figure 4.24: Level 3 SMT unpacking and 2D clustering time for real data (barrels only) 


\section{Chapter 5}

\section{Central Fibre Tracker readout software}

\subsection{CFT electronics and readout}

The readout system for the fibre tracker has many similarities with the silicon tracker system described in section 4.1 , but also a number of significant differences. A (now somewhat outdated) description of the electronics and readout is given in reference [70].

\subsubsection{CFT readout}

Light from the scintillating fibres is taken by optical waveguides to the Visible Light Photon Counters (VLPCs) located in the platform below the detector. The VLPCs are silicon diode devices which convert single photons to an amplified electrical pulse with a high quantum efficiency [71]. The digitisation of this signal is carried out by SVXIIe chips of the same type as used in the SMT readout, but these are too slow to provide a usable signal for the Level 1 trigger. For this reason each channel also 
produces a discriminated output on every bunch crossing. In the event of a Level 1 trigger accept, the analogue signal is digitised by the SVX chip (the signal from each crossing is held in a pipeline within the chip, as for the SMT readout).

The discriminator signal is provided by a SIFT (Scintillating Fibre Trigger) chip. Four of these SIFTs are packaged together with one SVX chip to form a Multi-Chip Module (MCM). Each MCM has a total of 72 channels, although only 64 of them are actually used in the readout. Eight MCMs make up an Analogue Front End (AFE) board [72]. (The discriminator signals for each bunch crossing are sent to the Digital Front End boards, which are part of the Level 1 track trigger, but this will not be discussed here.)

The digitised signals created on each Level 1 trigger accept are sent from the AFE boards to Sequencer boards, and from the Sequencers via a fibre optic link to VRBs located outside the collision hall in exactly the same way as for the SMT readout. A difference is that there are no HDIs in the fibre tracker system; however, the SVX chips in an AFE are connected as a chain in a configuration very similar to that in an HDI. Any reference to an HDI with respect to the CFT readout should be taken as a reference to an SVX chain instead.

Figure 5.1 shows the components of the CFT readout (compare with figure 4.1 for the SMT readout).

The existing design of the MCM and SIFT electronics is not suitable for operation with the 132 ns bunch crossing time [73]. The current MCMs will be replaced by this point (see section 1.2, page 17) with a new version which will subsume both the Virtual SVX and the normal SVX chips [38, 74]. Some or all of the following information on the raw data and unpacking of CFT data will therefore become obsolete when this happens. 


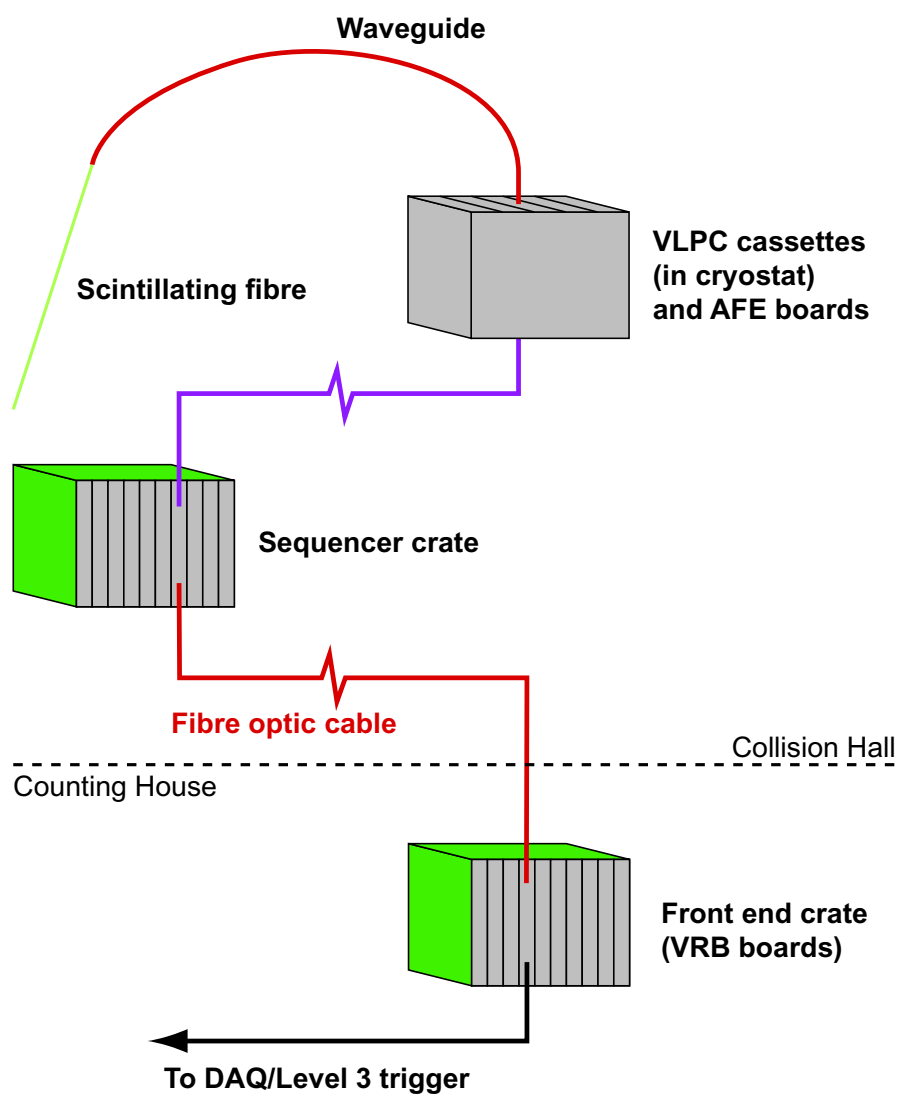

Figure 5.1: Diagram of the CFT readout system

\section{Virtual SVX}

In addition to the normal SVX chips, a chain can also contain a Virtual SVX chip (VSVX) [75]. A VSVX appears to the Sequencer and the rest of the readout as a normal SVX chip, but instead of the normal sequence of channel/ADC count data words it can be programmed to emit an arbitary stream of data into the readout. The Virtual SVX is used to insert the discriminator bits from each AFE into the event data.

As far as the sequencer is concerned the Virtual SVX chip looks exactly the same as a normal SVX chip. This means that the DAQ hardware from the sequencers onwards is exactly the same as for the silicon tracker. 


\subsubsection{CFT raw data format}

The format of the raw data from the CFT is almost identical to that from the SMT described in section 4.2 (but with the substitution of chains for HDIs). The most significant difference is the addition of the VSVX containing the discriminator bits. In format the VSVX resembles an ordinary SVX chip added at the begining of the data for a chain. An $8 \mathrm{MCM}$ chain therefore contains nine chips' worth of data, arranged as follows:

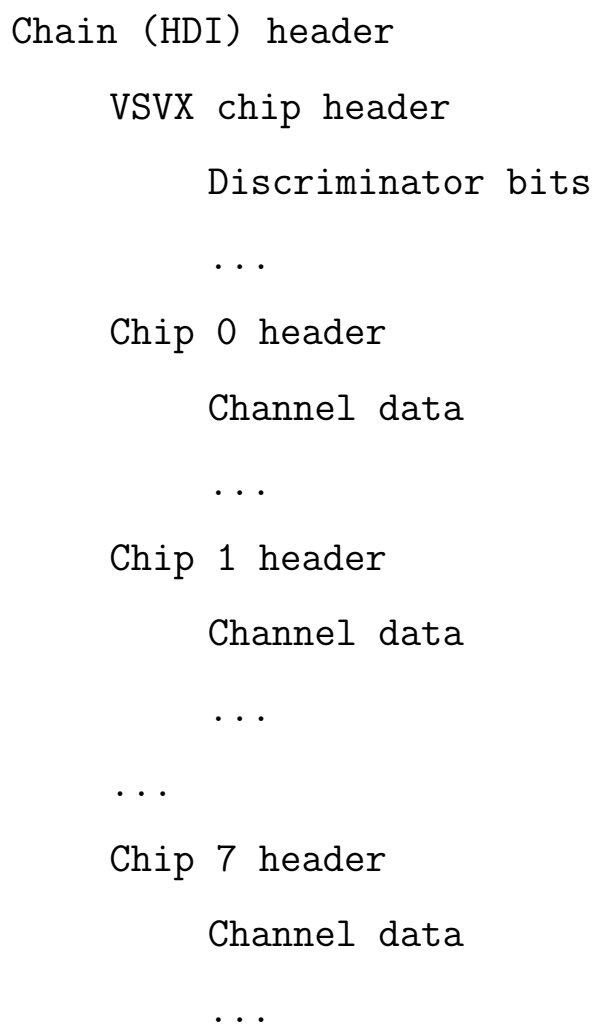

Chain (HDI) end of record

\section{VSVX data}

The VSVX has a chip ID of $0 x F$, which is a reserved value not allowed for normal SVXs. The format of the VSVX chip header word is: 


15
\begin{tabular}{|l|l|l|l|l|l|l|l|l|}
\hline 1 & 0 & 0 & 0 & 1 & 1 & 1 & 1 & \\
\hline
\end{tabular}

Unlike the ordinary SVX header described on page 65, the lower data byte of the VSVX header can be non-zero (for the details of the Status bits see reference [75] - currently the software makes no use of them $\left.{ }^{1}\right)$.

Like the normal data for an SVX chip, the VSVX data is divided up into words formed from an address byte and a data byte. Unlike the normal SVX, the data byte is not interpreted as a numeric value, but as a bit pattern, where each bit gives the discriminated value of a single fibre ${ }^{2}$. The upper four bits of the address byte give the number of the MCM which these discriminators belong to, and the lower four bits are a byte counter for the data within an MCM. Both of these values are in the range 0-7 - there are eight MCMs in an AFE chain, and each has 64 bits, or eight bytes, worth of data.

Each discriminator bit within an MCM corresponds to a single channel in the SVX chip for that MCM; however, the discriminator bits are in a different order from the SVX channels. Additionally the order of the data within the VSVX is not what might be expected - instead of the data for each MCM appearing in sequence they are mixed together:

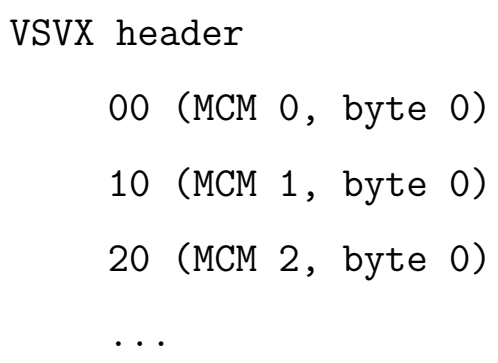

${ }^{1}$ Due to various design and specification changes, most of these flags are unused in the production version of the AFE board.

${ }^{2}$ The Gray decoding feature (see page 66 ) of the VRB would obviously spoil the bit pattern if applied to the VSVX data bytes. Fortunately the VRB logic is intelligent enough to ignore chips with the VSVX ID when doing the decoding. 


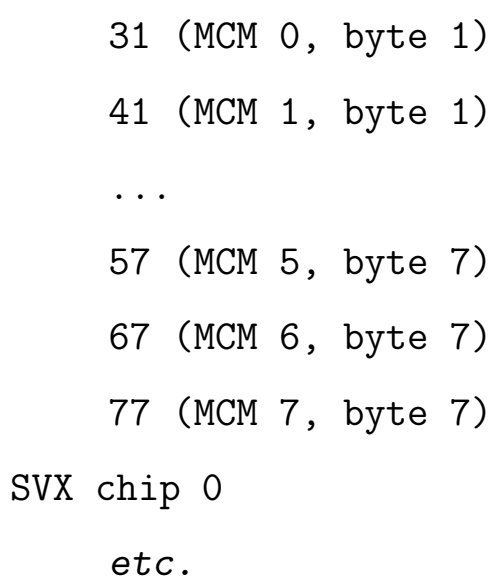

\section{SVX data}

The SVX data for the fibre tracker is identical in format and meaning to that for the silicon tracker, as described in section 4.2.2. Only 64 channels in each SVX chip are used - these are not a continuous block of channel numbers, but a seemingly random scattering throughout the 128 available on the chip (this is due to restrictions on the layout of the MCM board).

\section{$5.2 \quad$ Unpacking software}

The software for interpreting the CFT raw data works in a very similar way to that for the SMT. The only difference is the requirement to deal with the VSVX containing the discriminator data. The Level 3 trigger and the offline reconstruction differ in their treatment of the VSVX. The offline code unpacks the discriminator bits, while the trigger code just skips over them.

\subsubsection{Offline unpacking}

Like the SMT offline unpacking, the CFT unpacker is in two parts: the first converts the raw data to an UnpDataChunk, and the second converts the UnpDataChunk to a 
list of struck fibres in an CFTDigiChunk. Only the first part is described here.

The VRB header and HDI (in this case chain) headers are handled in the same way as with the SMT data (see section 4.3.2 on page 67).

Handling the data is slightly more complicated, as each discriminator bit corresponds to an ADC channel, and the two should be matched together. The lack of ordering in the VSVX channels is a problem here, so the method employed is to loop through the VSVX chip first, converting each discriminator bit to its equivalent SVX channel ID. When all the set bits within the VSVX have been read, then the channel ID lists for each MCM are sorted into numerical order so they match the order in the SVX readout.

Unpacking the remaining SVX chips now proceeds as for the SMT. However it simultaneously loops through the list of channels from the discriminators as well as from the SVX data, so that each channel can be stored in the UnpDataChunk in the correct order with the correct state (discriminator fired only, ADC count only, or both $^{3}$ ). Figure 5.2 shows a flowchart for the algorithm.

\subsubsection{Level 3 trigger unpacking}

Like the Level 3 SMT unpacking tool, the CFT unpacking tool both unpacks the raw data and clusters the fibres. However the time gain is less for the fibre tracker as the fibres are not read out in order. Because of this, all the fibres must be read out from the data first, sorted into order, and then clustered. The discriminator data is ignored within the trigger, and only the ADC values are used. Currently, fibres are just considered as either struck or not, so the only consideration is whether the ADC count is above some threshold or not.

\footnotetext{
${ }^{3}$ In theory the only case that should occur is 'both' — if a fibre is struck then it should be over the discriminator threshold and the SVX zero-supression readout threshold. But since the discriminator threshold is analogue and the SVX readout threshold is digital they are unlikely to match exactly, and there will be dead or noisy channels, electronics noise, etc., so one could be active without the other.
} 


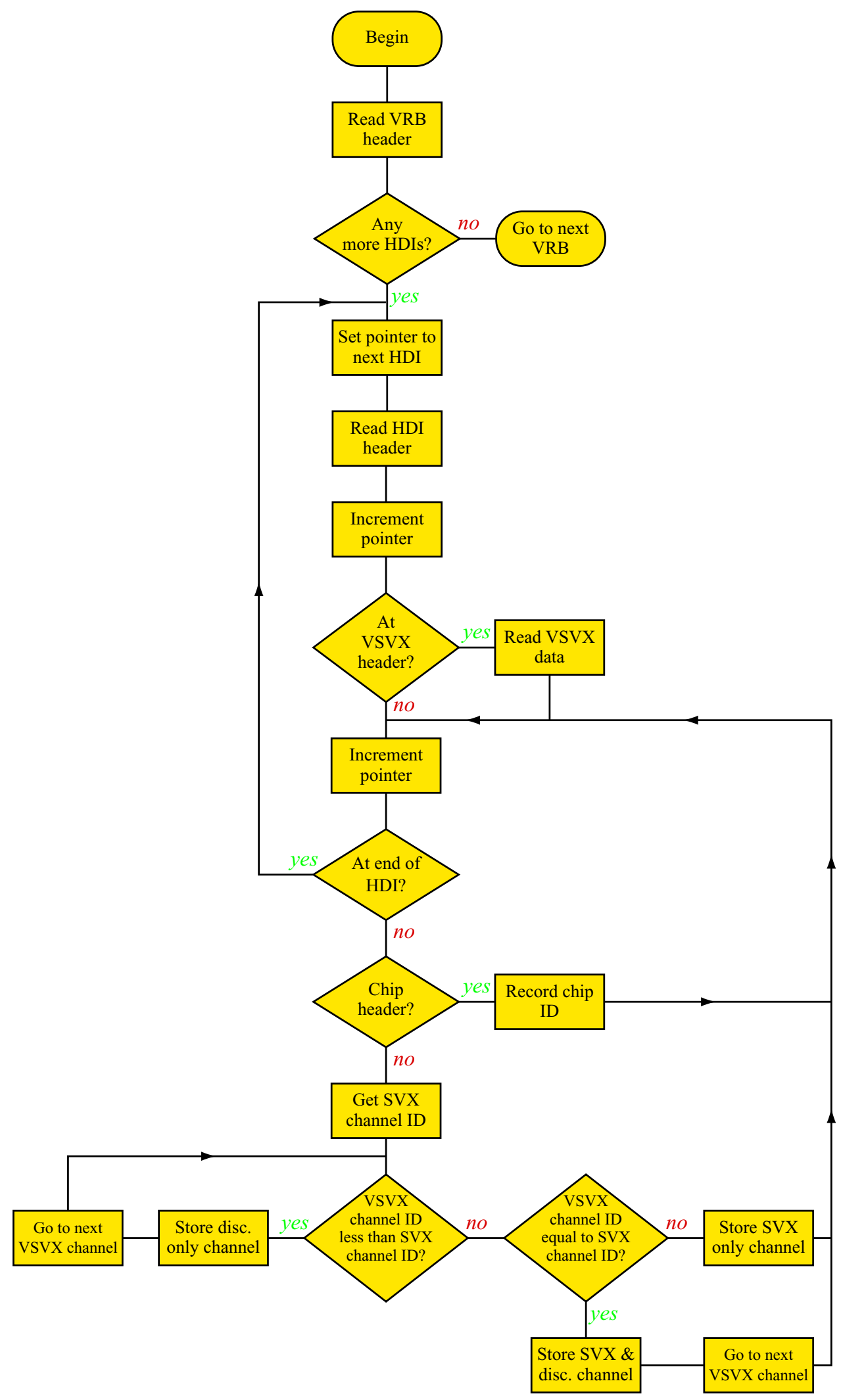

Figure 5.2: Flowchart for the CFT offline unpacking algorithm 


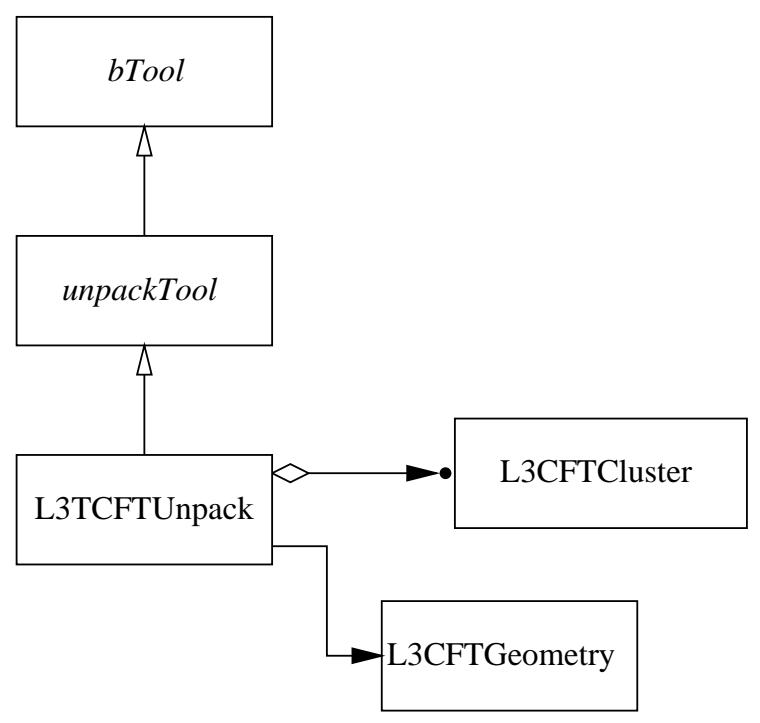

Figure 5.3: The class design of the Level 3 CFT unpacking and clustering tool

In the future it may be possible to use the ADC count to perform pulse height weighted clustering in a similar way to the silicon tracker. However due to the more complicated geometry of a fibre doublet, simply weighting the average fibre position by the pulse height will not work ${ }^{4}$, and improving the resolution this way is much harder than for the silicon detector.

Figure 5.3 shows the class design of the tool. (The key to the symbols in the diagram can be found in figure 4.5 on page 71 .)

\subsection{Level 3 trigger clustering}

The CFT consists of 16 concentric barrels of fibre doublet layers (see section 3.2.3 and figure 3.2). The fibres are numbered from 1 in increasing $\phi$-coordinate order within each barrel. Once the unpacked fibres have been sorted into order the clusters

\footnotetext{
${ }^{4}$ In a silicon detector the pulse height weighting works because of charge sharing between adjacent strips. For a scintillating fibre there is no charge sharing effect between adjacent fibres - instead the size of the pulse should depend on the amount of active material in the fibre the charged particle has traversed. The relative pulse height between two fibres will depend strongly on the angle with which the track crosses the fibre layer as well as its position. Additionally, the pulse height will be subject to fluctuations in the number of photoelectrons received by the VLPC.
} 
are formed by grouping adjacent fibres together. Since the fibres form a continuous layer a single cluster can cross the discontinuity in the fibre numbering and will therefore be split into two separate clusters (for example, a single particle can strike both fibres 3520 and 1 on the outermost layer and so initially appear as two separate clusters). If this happens the two clusters are merged together into a single cluster.

\subsubsection{Results}

The clusters formed by the Level 3 CFT unpacking tool are used by the tracking tool. Figure 5.4 shows a Monte Carlo event reconstructed by the CFT only tracking tool [76]. Figure 5.5 shows the track - cluster residuals for the reconstructed tracks. The fitted function is the sum of two Gaussian distributions, and the narrow peak has $\sigma=91 \mu \mathrm{m}$. This compares with cosmic ray tests of fibre doublet layers, which obtained a track resolution of $92 \mu \mathrm{m}[3]$.

During the accelerator shutdown in October and November 2001 the readout boards for all axial fibres (and part of the stereo fibres) were installed. Figure 5.6 shows tracks found in an event from a run taken on 1 December 2001. The readout electronics were still being debugged on this date, so there was a large amount of noise producing fake clusters present. The Level 3 unpacking and clustering tool and the CFT tracking tool were able to find a number of tracks, including some with hits on all eight axial layers. 


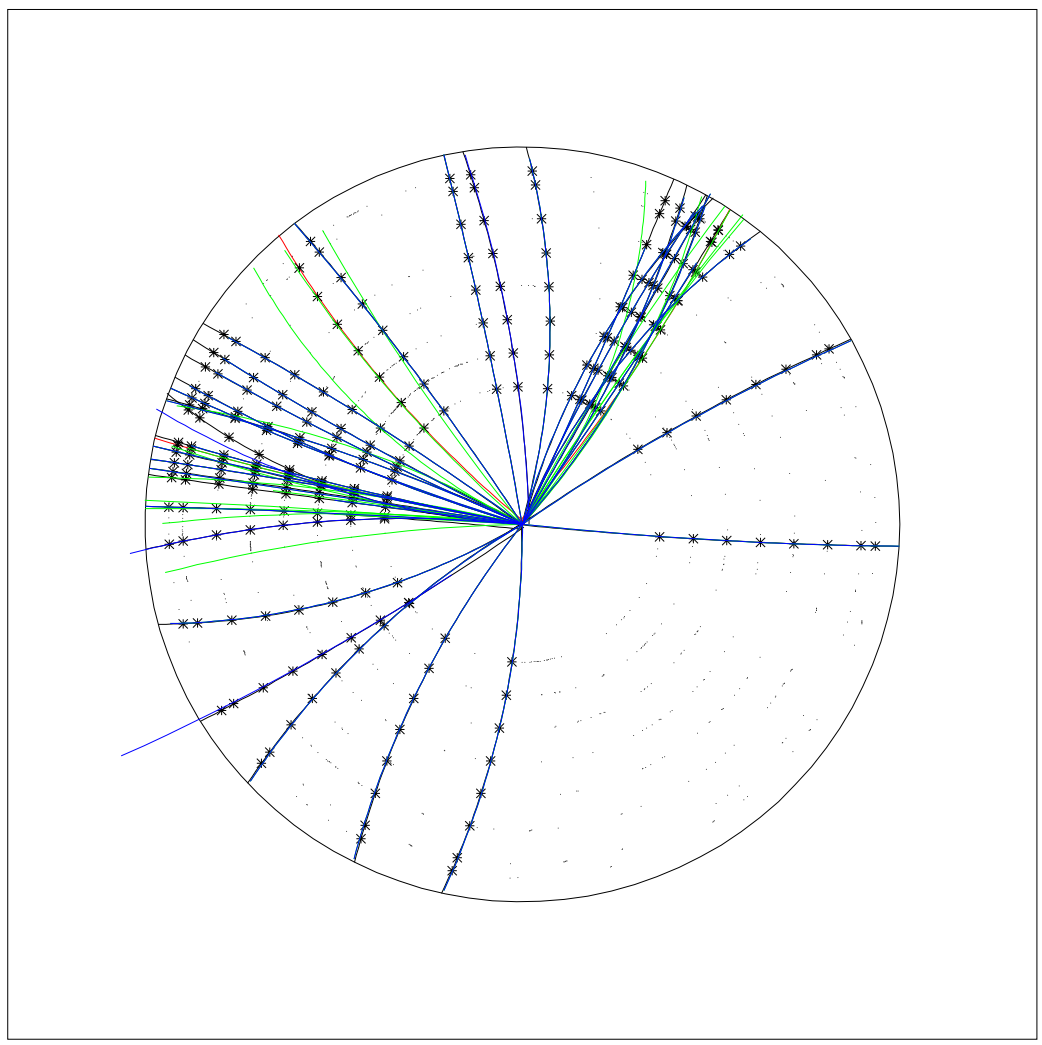

Figure 5.4: Monte Carlo $t \bar{t}$ event reconstructed with the Level 3 CFT tracker [77]

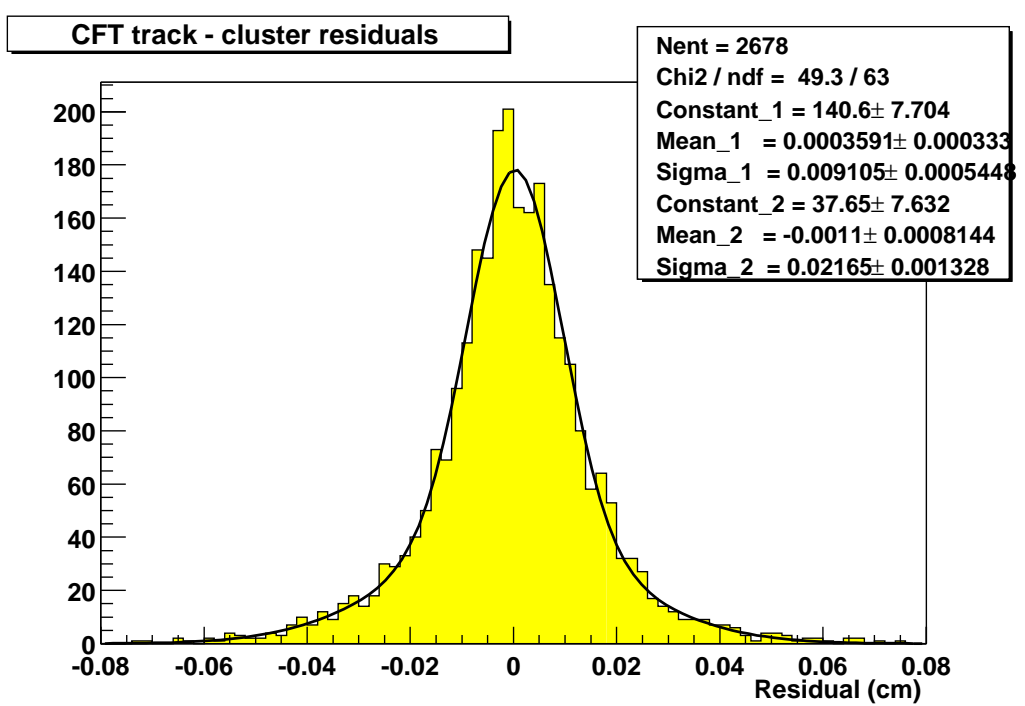

Figure 5.5: CFT track - cluster residuals for axial clusters. The residual distance is calculated as $r\left(\phi_{\text {cluster }}-\phi_{\text {track }}\right)$ where $r$ is the radius from the beam line of the cluster, $\phi_{\text {cluster }}$ is the $\phi$ coordinate of the cluster, and $\phi_{\text {track }}$ is the $\phi$ coordinate of the track propogated to radius $r$. 


\section{Run 139930 Event 187364}

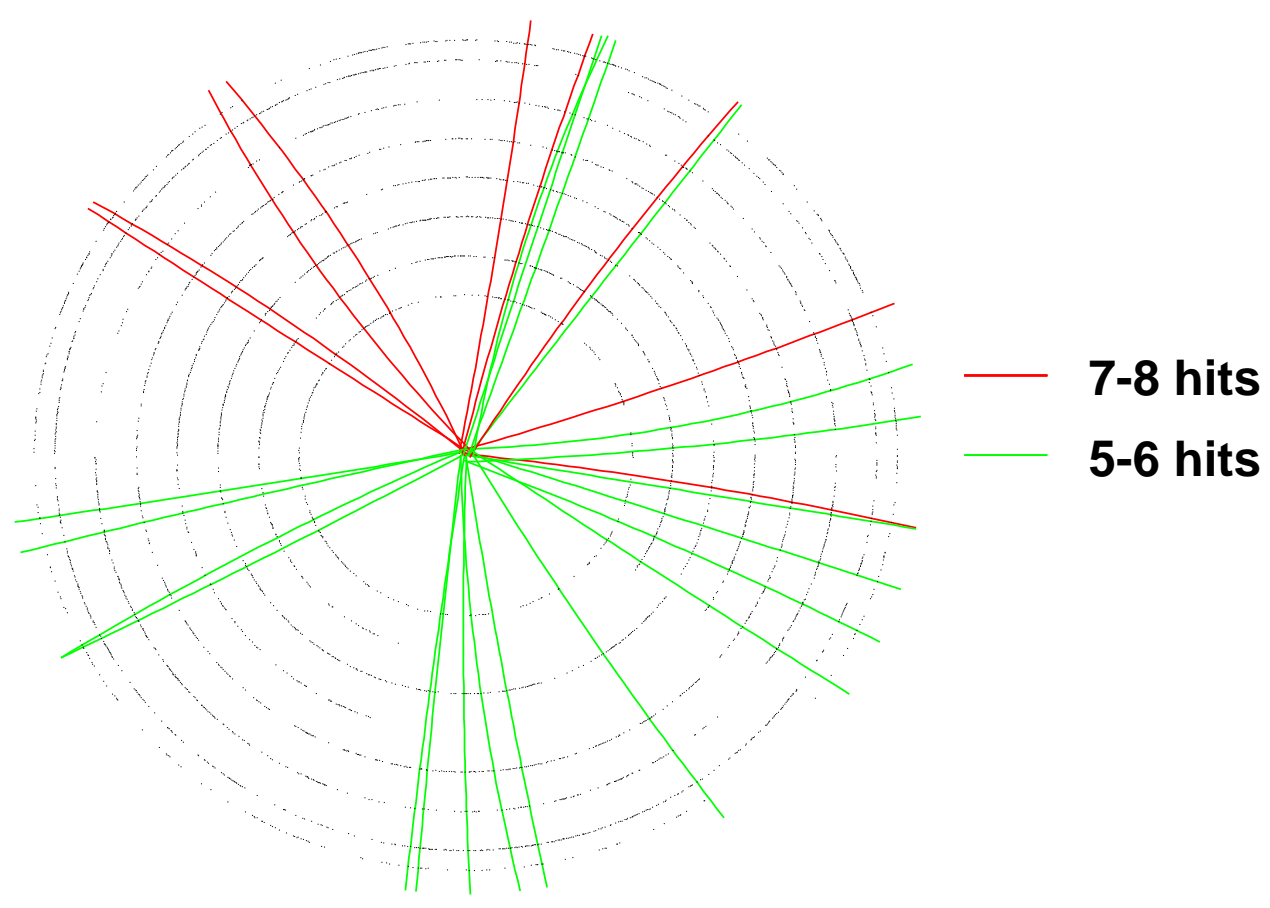

Figure 5.6: Event display from running the CFT unpacking, clustering, and tracking on early data (taken 1 December 2001) with the full axial CFT instrumented and being read out 


\subsubsection{Timing results}

Figure 5.7 shows the time taken to unpack and cluster a variety of different Monte Carlo event types and figure 5.8 shows the dependence on the number of minimum bias interactions in the event. These can be compared with the similar plots for the silicon detector in section 4.6 on page 87 . Compared to the silicon, the CFT has a much lower level of "overhead" in its scaling with the number of minimum bias interactions - this is because it has both a smaller number of readout crates to unpack, and a much simpler geometry for the clustering, than the silicon.

The Monte Carlo for the CFT does not include simulating the noise in the fibre tracker readout. To show the effect of noise, figure 5.9 plots the mean tool running time for varying levels of randomly added channels. Even a large number of extra channels does not increase the time to an unreasonable level.

Finally, figure 5.10 shows the time taken to unpack and cluster a sample of real data events from the detector. This is significantly greater than the Monte Carlo times. At this point in time, the fibre tracker electronics were still being commissioned, and there were a number of significant problems with some parts of the data, such as incorrect VRB lengths, missing HDI end of record markers on every event, invalid chip IDs, and corrupted data from some HDIs. Because of these problems the current performance does not necessarily show the running time when the detector is fully operational. 

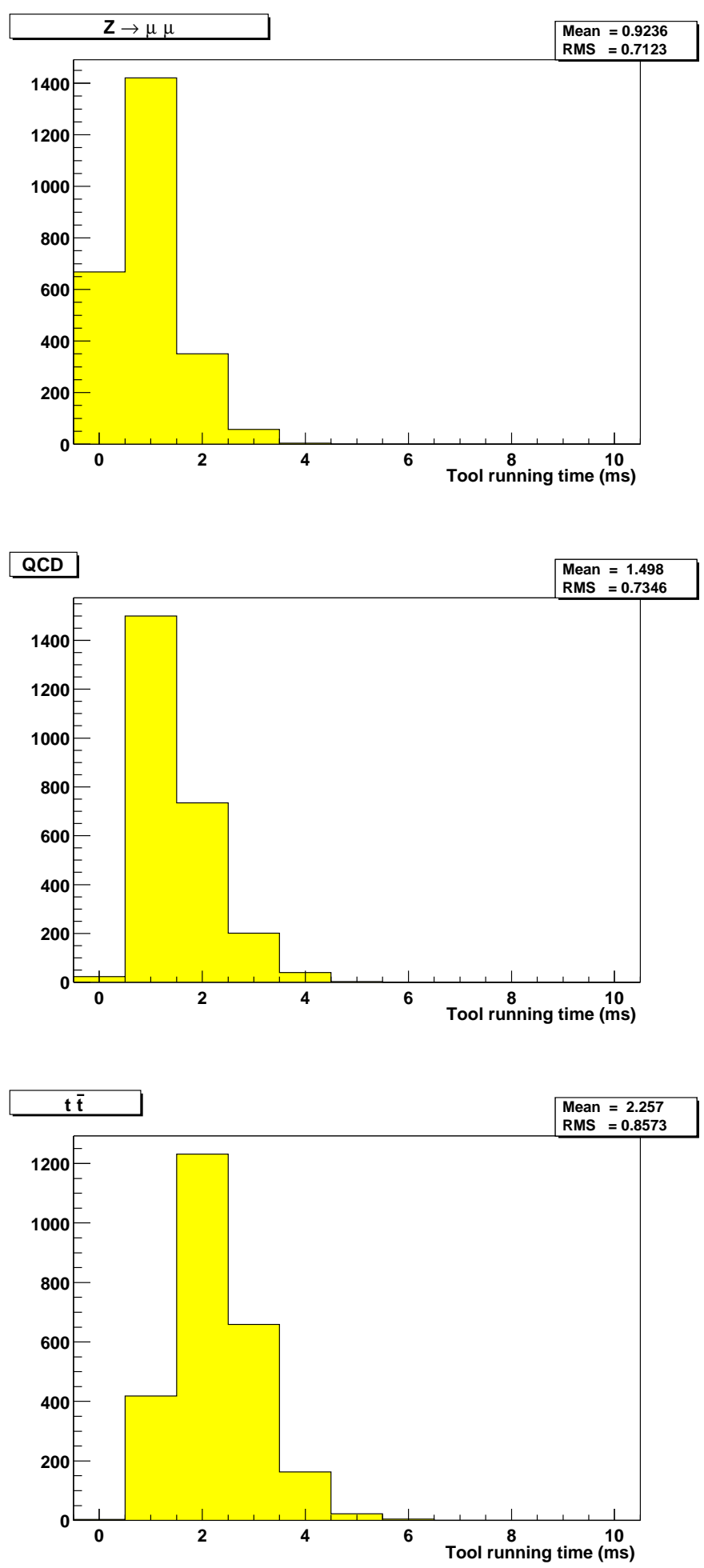

Figure 5.7: CFT unpacking and clustering time for different Monte Carlo event types. 


\section{CFT unpacking/clustering time for $\mathbf{Z} \rightarrow \mu \mu$}

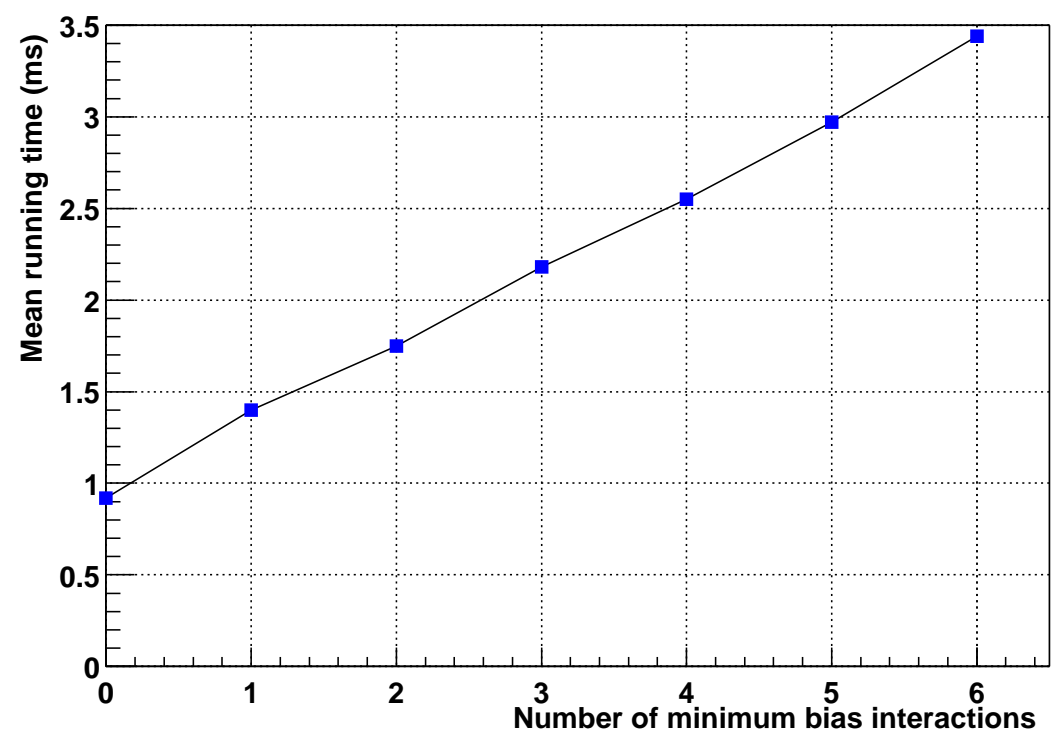

Figure 5.8: The unpacking and clustering time for $Z \rightarrow \mu \mu$ events with varying numbers of added minimum bias interactions.

\section{CFT unpacking/clustering time}

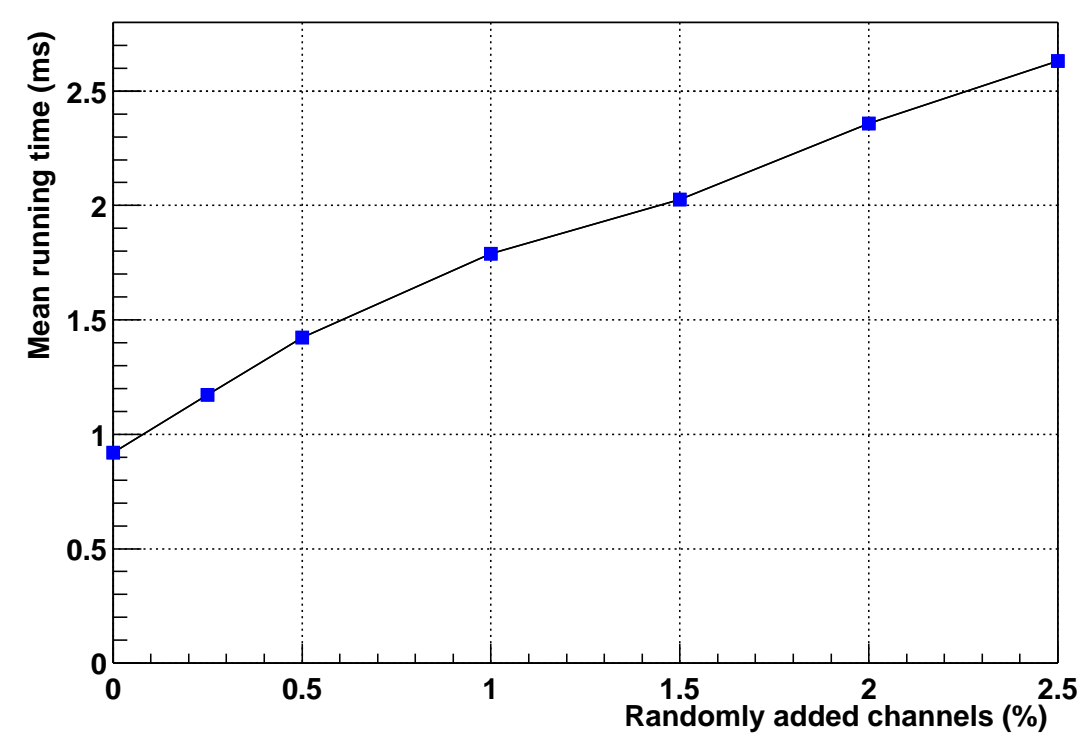

Figure 5.9: The unpacking and clustering time for $Z \rightarrow \mu \mu$ events with varying levels of added noise 


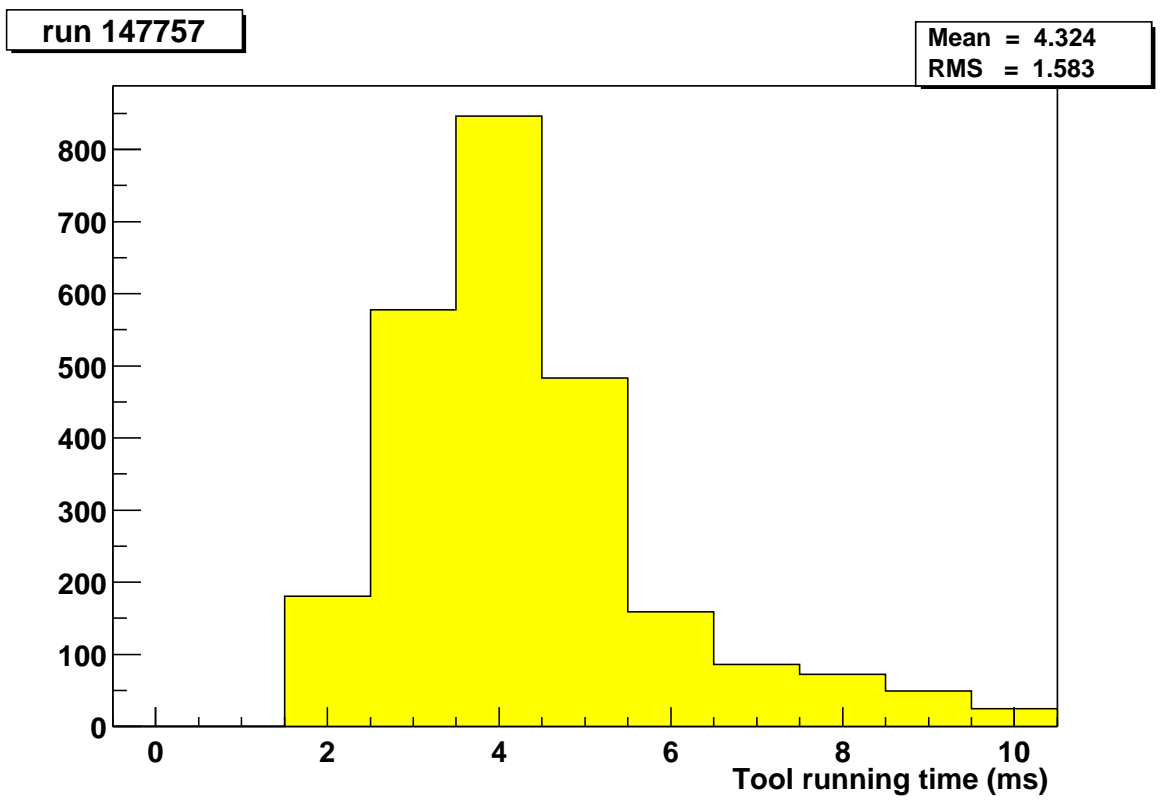

Figure 5.10: Level 3 CFT unpacking and clustering time for real data 


\section{Chapter 6}

\section{Mass constrained vertex fits}

\subsection{Mass constrained fit}

A mass constrained fit uses the hypothesis that a reconstructed particle has a particular mass to improve the values of its track parameters. For example, if a pair of oppositely charged muon tracks has an invariant mass close to that of the $J / \psi$ mass, then the track parameters can be recalculated under the assumption that the mass should be exactly that of the $J / \psi$. Where the $J / \psi$ is then used to reconstruct a heavier particle, the constrained fit will usually improve the momentum resolution of this heavier particle.

Consider the case of $n$ tracks, where the tracks have been constrained to a common vertex $[78,79]$, and the 3 -momentum of each track is known at the vertex point.

The initial values of all the track and vertex parameters are

$$
\boldsymbol{\alpha}_{0}=\left(\begin{array}{c}
\mathbf{p}_{0} \\
\vdots \\
\mathbf{p}_{n} \\
\mathbf{x}
\end{array}\right)
$$


where

$$
\mathbf{p}_{i}=\left(\begin{array}{c}
p_{i x} \\
p_{i y} \\
p_{i z}
\end{array}\right) \quad \text { and } \quad \mathbf{x}=\left(\begin{array}{c}
x \\
y \\
z
\end{array}\right)
$$

are the initial momentum of each track and the vertex position ${ }^{1}$.

The mass constrained parameters can be found by minimising the value of the $\chi^{2}$ statistic $[80]$

$$
\chi^{2}=\left(\boldsymbol{\alpha}-\boldsymbol{\alpha}_{0}\right)^{T} V_{\boldsymbol{\alpha}_{0}}^{-1}\left(\boldsymbol{\alpha}-\boldsymbol{\alpha}_{0}\right)
$$

subject to the constraint ${ }^{2}$

$$
H(\boldsymbol{\alpha})=E^{2}-p_{x}^{2}-p_{y}^{2}-p_{z}^{2}-m^{2}=0
$$

where $\boldsymbol{\alpha}$ are the new values of the parameters, $V_{\boldsymbol{\alpha}_{0}}$ is the initial covariance matrix (which can include correlations between the momenta of different tracks, and between the momentum of each track and the vertex position), $E, p_{x}, p_{y}, p_{z}$ are the components of the sum of the 4-momenta of the tracks, and $m$ is the value to which the mass is to be constrained.

The value of $E_{i}$ is not a free parameter, but depends on the momentum and the (assumed) mass of the track

$$
E_{i}=\sqrt{p_{i x}^{2}+p_{i y}^{2}+p_{i z}^{2}+m_{i}^{2}}
$$

Minimising the $\chi^{2}$ can be done by linearising the constraint equations about the

\footnotetext{
${ }^{1}$ The vertex position is not directly affected by the mass constraint. But if the tracks have been constrained to the vertex then their momenta will be correlated with the position, and so the vertex position (and its error) will be changed indirectly.
}

${ }^{2}$ With $c=1$ throughout. 
initial track parameters and then using the method of Lagrange multipliers [80, 81]. Expanding about the initial values of the parameters reduces the constraints to

$$
\frac{\partial H\left(\boldsymbol{\alpha}_{0}\right)}{\partial \boldsymbol{\alpha}}\left(\boldsymbol{\alpha}-\boldsymbol{\alpha}_{0}\right)+H\left(\boldsymbol{\alpha}_{0}\right) \equiv \mathbf{D} \delta \boldsymbol{\alpha}+d=0
$$

where

$$
\begin{aligned}
\mathbf{D} & =\left(\begin{array}{llllll}
\frac{\partial H}{\partial p_{0 x}} & \cdots & \frac{\partial H}{\partial p_{n z}} & 0 & 0 & 0
\end{array}\right) \\
& =\left(\begin{array}{llllll}
2\left(E \frac{p_{0 x}}{E_{0}}-p_{x}\right) & \cdots & 2\left(E \frac{p_{n z}}{E_{n}}-p_{z}\right) & 0 & 0 & 0
\end{array}\right), \\
\delta \boldsymbol{\alpha} & =\boldsymbol{\alpha}-\boldsymbol{\alpha}_{0}, \quad \text { and } \\
d & =E^{2}-p_{x}^{2}-p_{y}^{2}-p_{z}^{2}-m^{2}
\end{aligned}
$$

The $\chi^{2}$ is now

$$
\chi^{2}=\delta \boldsymbol{\alpha}^{T} V_{\boldsymbol{\alpha}_{0}}^{-1} \delta \boldsymbol{\alpha}+2 \lambda(\mathbf{D} \delta \boldsymbol{\alpha}+d)
$$

where $\lambda$ is the Lagrange multiplier. The minimum is when

$$
\frac{\partial \chi^{2}}{\partial \boldsymbol{\alpha}}=V_{\boldsymbol{\alpha}_{0}}^{-1}\left(\boldsymbol{\alpha}-\boldsymbol{\alpha}_{0}\right)+\mathbf{D}^{T} \lambda=0
$$

so the new track parameters are given by

$$
\boldsymbol{\alpha}=\boldsymbol{\alpha}_{0}-V_{\boldsymbol{\alpha}_{0}} \mathbf{D}^{T} \lambda
$$

and combining equations (6.6) and (6.11) gives

$$
\begin{aligned}
\lambda & =\left(\mathbf{D} V_{\boldsymbol{\alpha}_{0}} \mathbf{D}^{T}\right)^{-1} d \\
& =V_{\mathbf{D}} d \quad \text { with } \quad V_{\mathbf{D}} \equiv\left(\mathbf{D} V_{\boldsymbol{\alpha}_{0}} \mathbf{D}^{T}\right)^{-1}
\end{aligned}
$$


The new covariance matrix is given by

$$
\begin{aligned}
V_{\alpha} & =\left\langle d \boldsymbol{\alpha} d \boldsymbol{\alpha}^{T}\right\rangle \\
& =\left\langle d \boldsymbol{\alpha}_{0} d \boldsymbol{\alpha}_{0}^{T}\right\rangle-V_{\boldsymbol{\alpha}_{0}} \mathbf{D}^{T}\langle d \lambda d \lambda\rangle \mathbf{D} V_{\boldsymbol{\alpha}_{0}} \\
& =V_{\boldsymbol{\alpha}_{0}}-V_{\boldsymbol{\alpha}_{0}} \mathbf{D}^{T} V_{\mathbf{D}} \mathbf{D} V_{\boldsymbol{\alpha}_{0}}
\end{aligned}
$$

This implies that the diagonal elements of the covariance matrix are reduced, so decreasing the errors on the particle momenta.

Finally, the $\chi^{2}$ of the fit is given by

$$
\begin{aligned}
\chi^{2} & =\delta \boldsymbol{\alpha}_{0}^{T} V_{\boldsymbol{\alpha}_{0}}^{-1} \delta \boldsymbol{\alpha}_{0} \\
& =\lambda \mathbf{D} V_{\boldsymbol{\alpha}_{0}} V_{\boldsymbol{\alpha}_{0}}^{-1} V_{\boldsymbol{\alpha}_{0}} \mathbf{D}^{T} \lambda \\
& =\lambda V_{\mathbf{D}}^{-1} \lambda \\
& =\lambda d
\end{aligned}
$$

The $\chi^{2}$ provides a useful measure of how compatible the original track parameters are with being forced to have a particular invariant mass. The mass constrained fit therefore has two purposes: it provides a method of rejecting combinations of tracks which do not actually come from a decaying particle; and for those combinations which are compatible with the decay of a particular particle it gives an improvement on the resolution of the track momenta.

\subsection{Results}

Figures 6.1, 6.2, and 6.3 show the effect of the mass constrained fit on the resolution of the momentum of reconstructed $J / \psi$ particles (decaying to $\mu^{+} \mu^{-}$). The Monte Carlo data used was from a sample of $B^{0} \rightarrow J / \psi K_{S}$ decays. The top plot of each figure is the resolution of the $J / \psi$ 's with only a vertex constrained fit, and the lower plot is the resolution with both the vertex and mass constrained fits. 


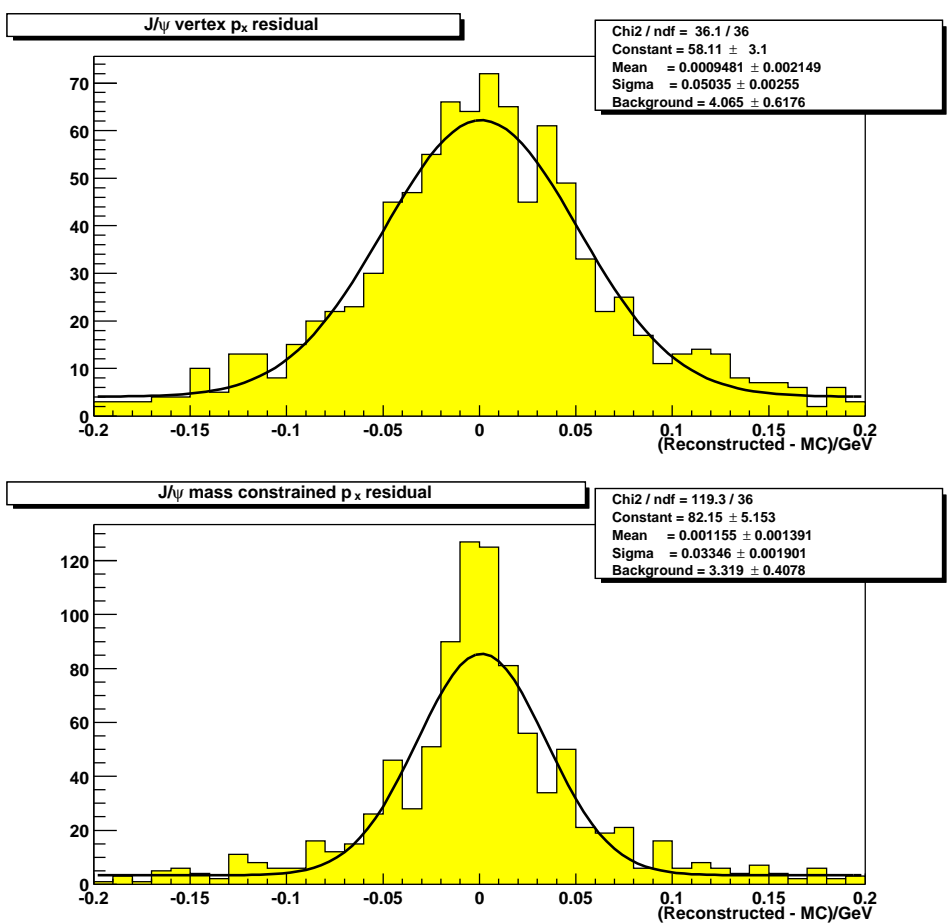

Figure 6.1: $x$-momentum residuals $\left(p_{\text {reconstructed }}-p_{\text {generated }}\right)$ for reconstructed Monte Carlo $J / \psi$

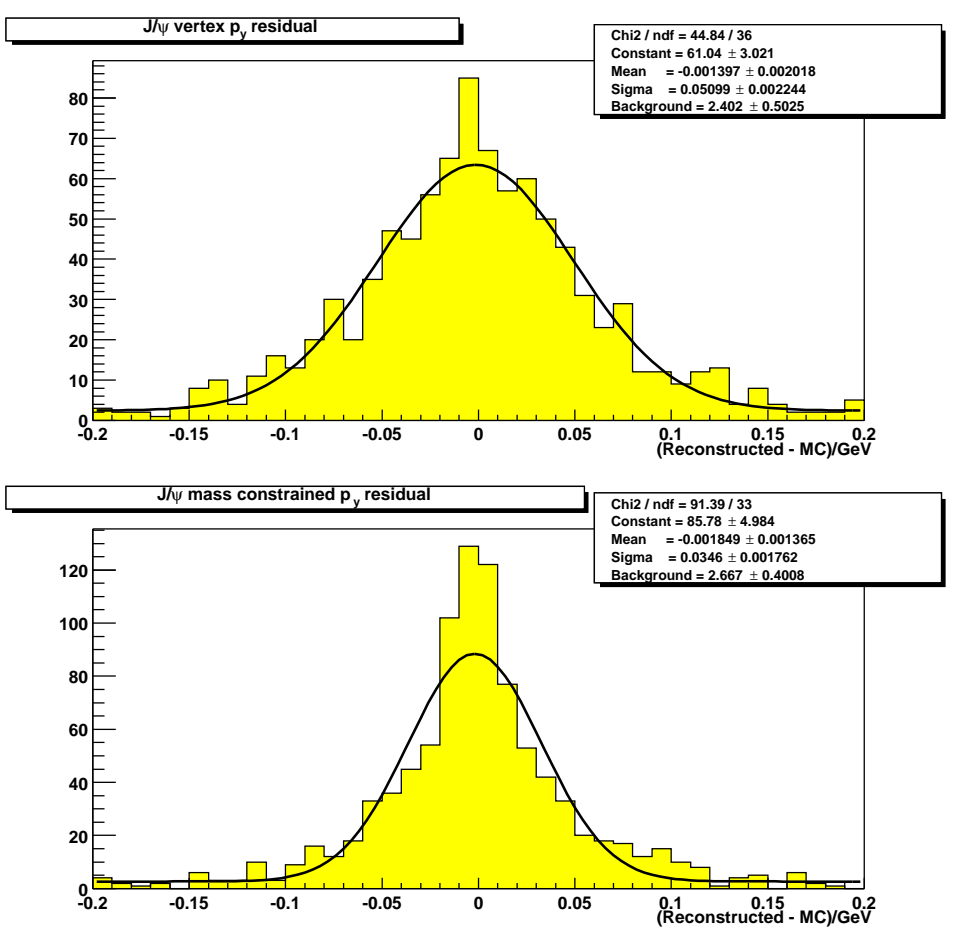

Figure 6.2: $y$-momentum residuals $\left(p_{\text {reconstructed }}-p_{\text {generated }}\right)$ for reconstructed Monte Carlo $J / \psi$ 


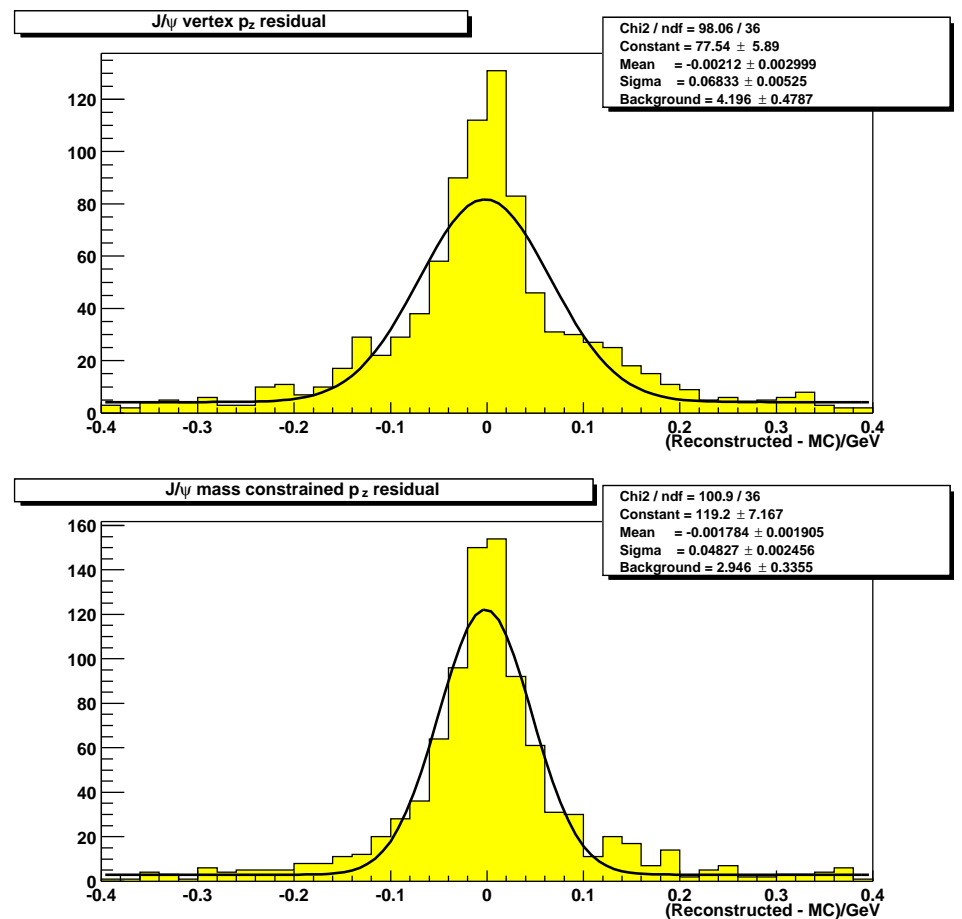

Figure 6.3: $z$-momentum residuals $\left(p_{\text {reconstructed }}-p_{\text {generated }}\right)$ for reconstructed Monte Carlo $J / \psi$

Figure 6.4 shows the pull distributions (reconstructed momentum minus Monte Carlo momentum, divided by the error) for the vertex constrained fits only, and figure 6.5 shows the pull distributions of the mass constrained fits. The pull distribution is expected to be Gaussian with $\sigma=1$. The values of fitted sigmas of the pulls for the vertex constrained fits are slightly high (particularly for the $z$-momentum) - this is due to problems with the errors on the original track fit parameters [82]. As the vertex constrained errors are used as input into the mass constrained fit the errors calculated by the fitting procedure will suffer due to this, but figure 6.5 shows that they still look reasonable (except for the $z$-momentum pull, which has too narrow a distribution, suggesting the errors are too large).

Despite this, the mass constraint has significantly improved the resolution of the $J / \psi$ momentum, bringing the $x$ and $y$ resolutions from $\sim 50 \mathrm{MeV}$ down to $35 \mathrm{MeV}$ or better. The $z$ momentum is less dramatic, but the tails of the residual distribution are significantly reduced. 

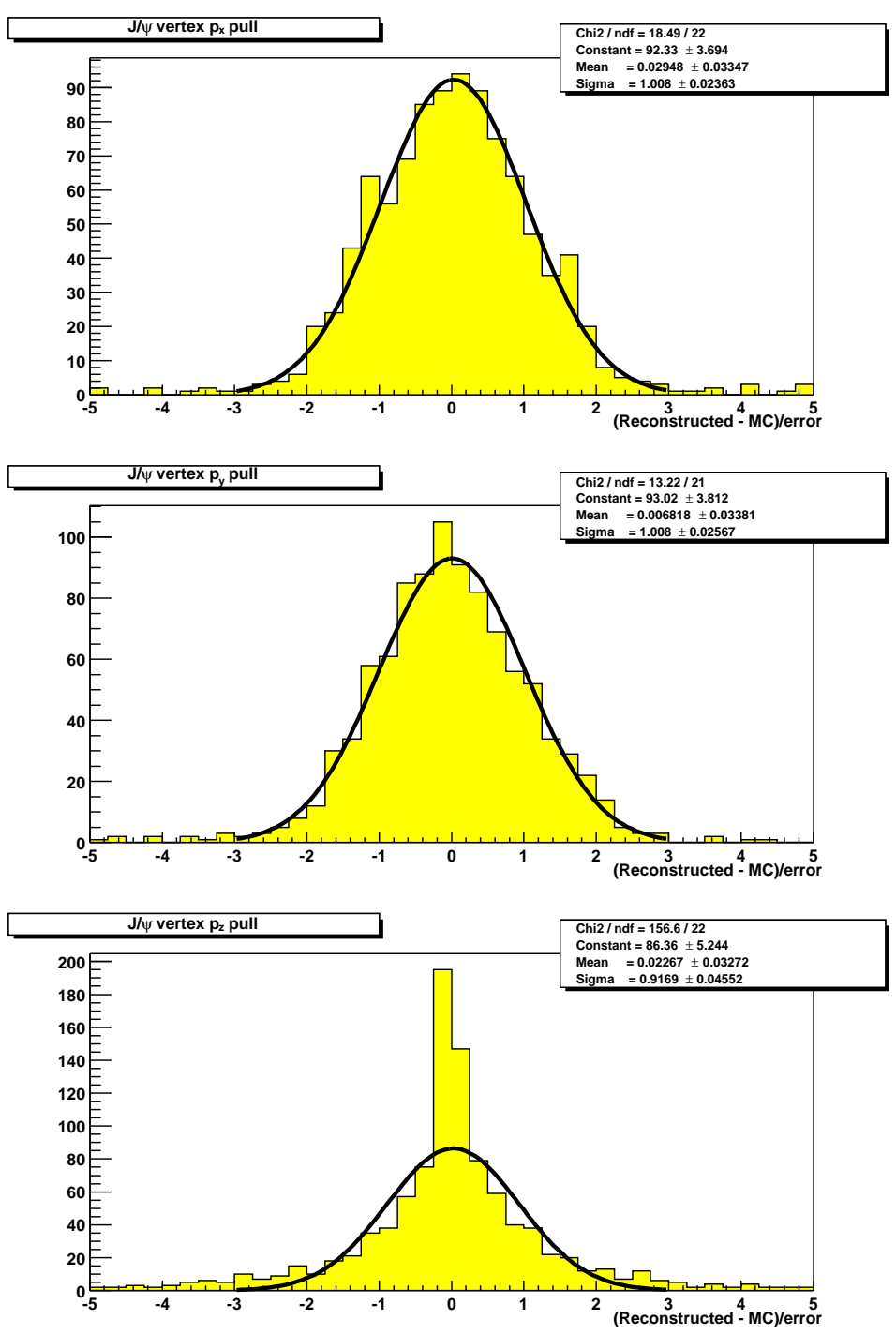

Figure 6.4: Momentum pulls $\left(p_{\text {reconstructed }}-p_{\text {generated }}\right) / \sigma_{p}$ for vertex fitted Monte Carlo $J / \psi$ 

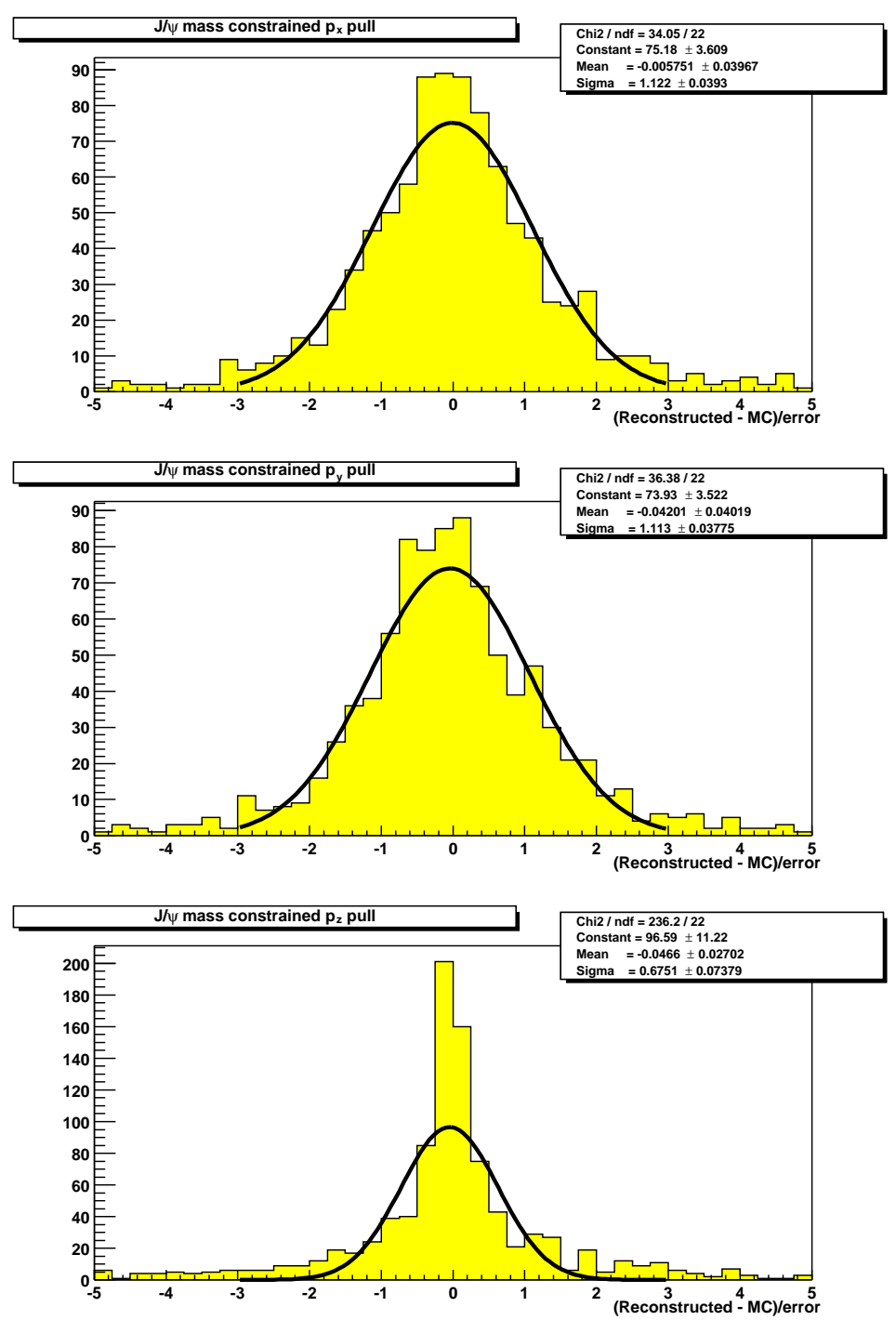

Figure 6.5: Momentum pulls $\left(p_{\text {reconstructed }}-p_{\text {generated }}\right) / \sigma_{p}$ for vertex fitted, mass constrained Monte Carlo $J / \psi$ 
The mass constrained fit is used in the following chapter, where mass constraining the muons coming from the decay of a $J / \psi$ is used to improve the momentum resolution of the $B^{+}$meson in a $B^{+} \rightarrow J / \psi K^{+}$decay. 


\section{Chapter 7}

\section{$B^{+}$lifetime}

\subsection{Introduction}

The measurement of the lifetimes of the different $B$ mesons and baryons is significant for two reasons. Firstly, the experimental measurements of the lifetimes can be compared to the predictions of the heavy quark expansion technique mentioned in section 2.6.2. Secondly, the techniques involved in measuring the lifetime are equally applicable to those required for measuring time dependent $\mathrm{CP}$ violation effects and $B^{0}-\bar{B}^{0}$ mixing, namely the event by event reconstruction of the $B$ decay vertex.

The main focus of this study was to look at measuring the lifetime of the $B^{+}$through the decay ${ }^{1} B^{+} \rightarrow J / \psi K^{+}, J / \psi \rightarrow \mu^{+} \mu^{-}$. This decay has the advantage of being easy to trigger on (using a dimuon trigger), and relatively easy to reconstruct. The reconstruction is done by first forming $J / \psi$ candidates, constraining the muon tracks to a common vertex position, and then adding single tracks (assumed to be $K^{+}$ tracks) to the $J / \psi$ candidates to form a three track vertex. It is also possible to use

${ }^{1}$ References to a charge state almost always imply a reference to the charge-conjugate state as well (the exceptions are certain CP violating processes). Everything about $B^{+} \rightarrow J / \psi K^{+}$is equally applicable to $B^{-} \rightarrow J / \psi K^{-}$. 
other $B^{+}$decay modes such as $J / \psi K^{*+}, \psi(2 S) K^{+}, \psi(2 S) K^{*+}$, with $K^{*+} \rightarrow K_{S} \pi^{+}$ and $\psi(2 S) \rightarrow J / \psi \pi^{+} \pi^{-}$but these are not considered here (for the methods used by the CDF collaboration for these channels see [83]).

\subsection{Monte Carlo data sample}

The data used to simulate the decay was generated using the PYTHIA [84] and QQ [85] Monte Carlo programs. PYTHIA is used to generate the majority of the processes occurring in the collision (including the creation of the $B$ mesons themselves), while QQ is used to simulate the decay of the $B$ mesons and their daughter particles. The events generated are then filtered by keeping only those events which contain the desired particles satisfying the required kinematic cuts. For the $B^{+}$sample, the cuts required the generated $p_{\mathrm{T}}$ of the $B^{+}$to be greater ${ }^{2}$ than $4 \mathrm{GeV}$, and the muons coming from the $J / \psi$ decay were required to have $p_{\mathrm{T}}>2 \mathrm{GeV},|\eta|<1.6$. The pseudorapidity cut kept the muons in the region covered by the fibre track trigger (this is the pseudorapidity region where tracks pass through all eight layers of the fibre tracker).

The output of the particle generation stage is then passed through the simulation of the D $\varnothing$ detector. This has two parts, the program DØgstar ${ }^{3}$, which is a GEANT [86] based simulation of the detector, and døsim, which handles digitisation (and generates the simulated raw data, using some of the components referred to in chapters 4 and 5).

\footnotetext{
${ }^{2}$ All coordinate references are to the standard D $\varnothing$ global coordinate system, which is a right handed coordinate system with the $z$ axis pointing in the direction of travel of the protons, and the $y$ axis pointing vertically upwards. Unless otherwise specified, transverse means transverse to the beam line, therefore in the $x y$ plane.
}

${ }^{3}$ Alleged to stand for 'D $\varnothing$ GEANT simulation of the total apparatus response'. 


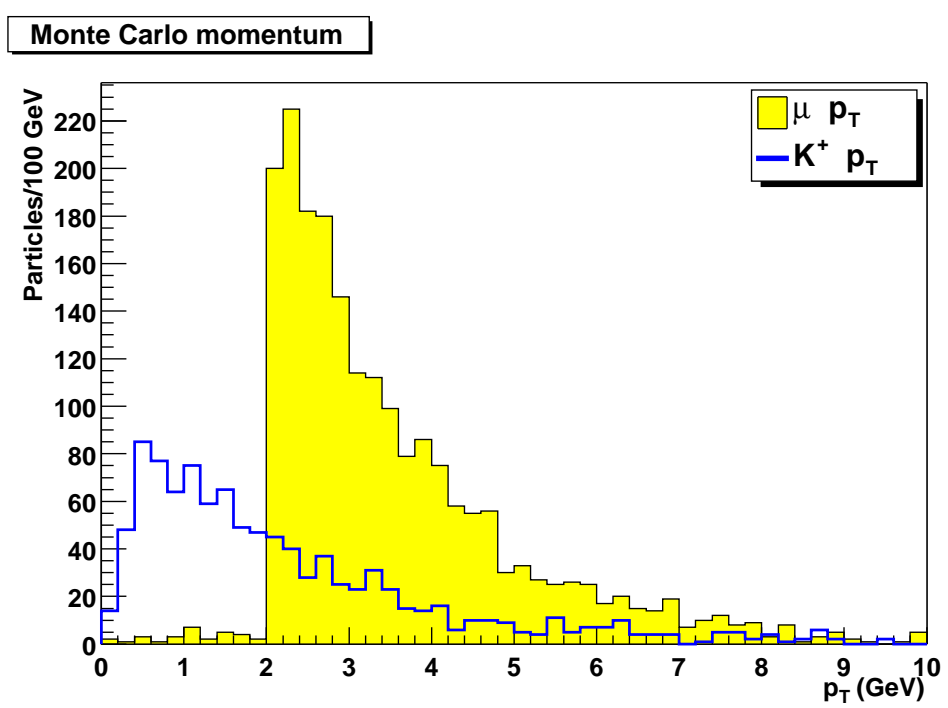

Figure 7.1: The Monte Carlo $p_{\mathrm{T}}$ spectrum of the muons and kaons coming from $B^{+}$decays. There was a generator level cut requring the two muons to each have $p_{\mathrm{T}}>2 \mathrm{GeV}$.

\section{$7.3 \quad J / \psi$ reconstruction}

The $J / \psi$ is reconstructed by combining oppositely signed charged tracks that come (or appear to come) from a common vertex. The $J / \psi$ is forced to decay to muons, but to allow for inefficiencies in the muon identification only one track is required to be matched to a muon track. The muon identification efficiency drops off significantly below $2 \mathrm{GeV}$, as at these energies there is a high probability that the muon will not traverse the full muon system. As figure 7.1 shows, there are a significant number of low $p_{\mathrm{T}}$ muons.

Each pair of opposite charge tracks with at least one track matched to a muon is constrained to come from a common vertex [79]. The $\chi^{2}$ statistic coming from the vertex constraint provides a way of rejecting tracks originating from different points.

Figure 7.2 shows the mass distribution from combining the tracks, vertex constraining them, and applying a cut on the vertex $\chi^{2}$ value by requiring $\chi^{2}<5$ (the two track vertex constraint has one degree of freedom, and the $\chi^{2}$ distribution is shown in figure 7.2(a)). Fitting a Gaussian for the peak plus a straight line background parameterisation gives a $J / \psi$ mass of $3.091 \pm 0.003 \mathrm{GeV}$ and a width of $0.047 \pm 0.003 \mathrm{GeV}$. 
This compares with the mass used in the simulation of $3.096 \mathrm{GeV}$.

When forming $B^{+}$candidates, only some of the $J / \psi$ candidates are used. The criteria for selecting $B^{+}$events are described in section 7.4 and figure 7.3 shows the invariant mass of only those $J / \psi$ candidates used to make $B^{+}$'s. In this case the fitted mass is $3.089 \pm 0.002 \mathrm{GeV}$ and the width is $0.042 \pm 0.002 \mathrm{GeV}$.

\subsubsection{Real $J / \psi$ s from data}

Figure 7.4 shows the $J / \psi$ invariant mass spectrum for muons detected in the muon system using data from the 5.8 million events taken between August and October 2001. During this time the fibre tracker was not operational, so the muon momenta are those measured by the muon tracking alone. Only muon candidates which had hits in all three layers of the muon system were used. The width of the fitted Gaussian distribution is $\sim 0.75 \mathrm{GeV}$.

\section{$7.4 \quad B^{+}$reconstruction}

Once the $J / \psi$ candidates have been found for an event, they are then used to search for the $B^{+}$candidates. The decay channel involved is $B^{+} \rightarrow J / \psi K^{+}$and the $K^{+}$ can be taken to be (on the scale of the $\mathrm{D} \varnothing$ detector) a stable particle, so the $B^{+}$will show up as a three track decay vertex. The procedure is to take each $J / \psi$ candidate, constrain its mass to the Particle Data Group value for the $J / \psi$ invariant mass [12] using the techniques of chapter 6 , then combine it with all the other tracks in the event, in each case performing a vertex constrained fit on all three tracks. The distribution of the $\chi^{2}$ for the vertex fit is shown in figure 7.5. Figure 7.6 shows the results of the candidate selection, with the only additional cut being $\chi^{2}<15$ on the three track vertex fit.

This procedure gives a number of $B^{+}$candidates for each event. This is then reduced to one candidate per event. First $J / \psi$ candidates formed from two muon tracks are 


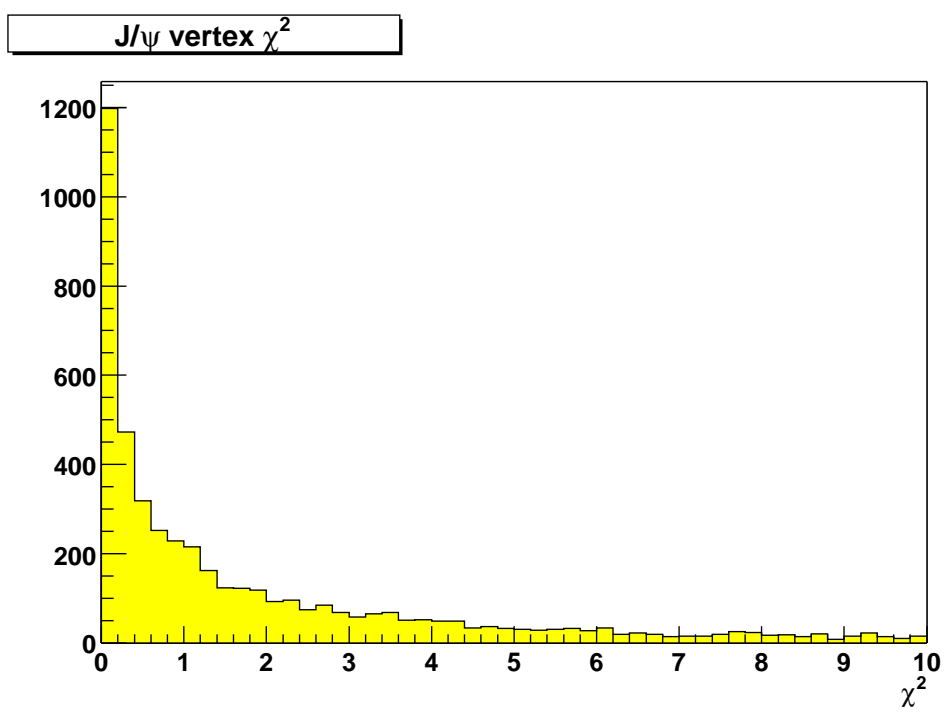

(a) $\chi^{2}$ distribution for the constrained vertex fit for $J / \psi$ candidates. The two track fit has one degree of freedom.

$\mathrm{J} / \psi$ invariant mass

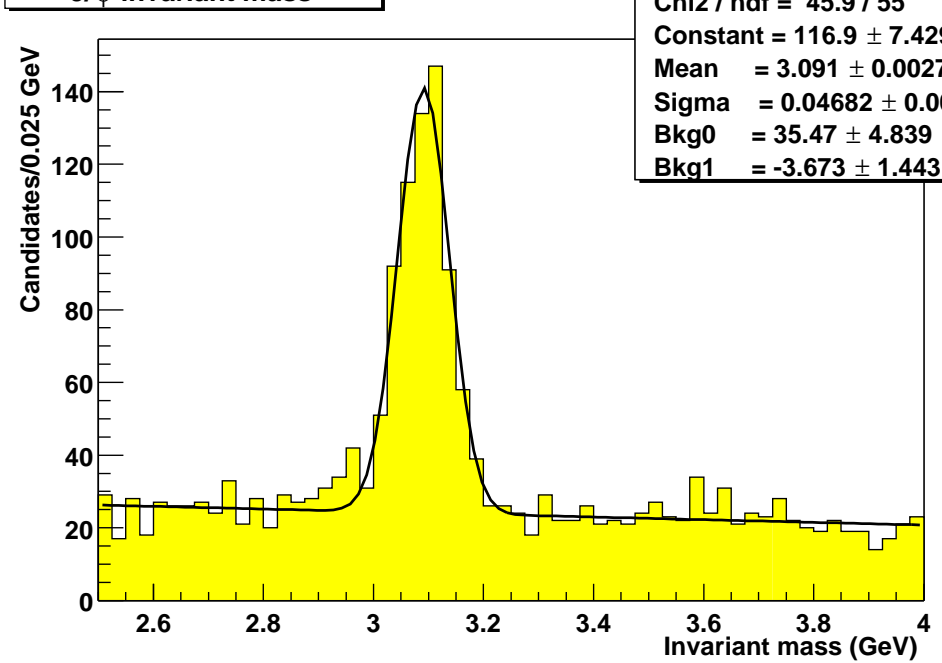

(b) Reconstructed $J / \psi$ mass, with fitted straight line background. A cut of $\chi^{2}<5$ was made on the vertex constrained fit.

Figure 7.2: $J / \psi$ invariant mass 


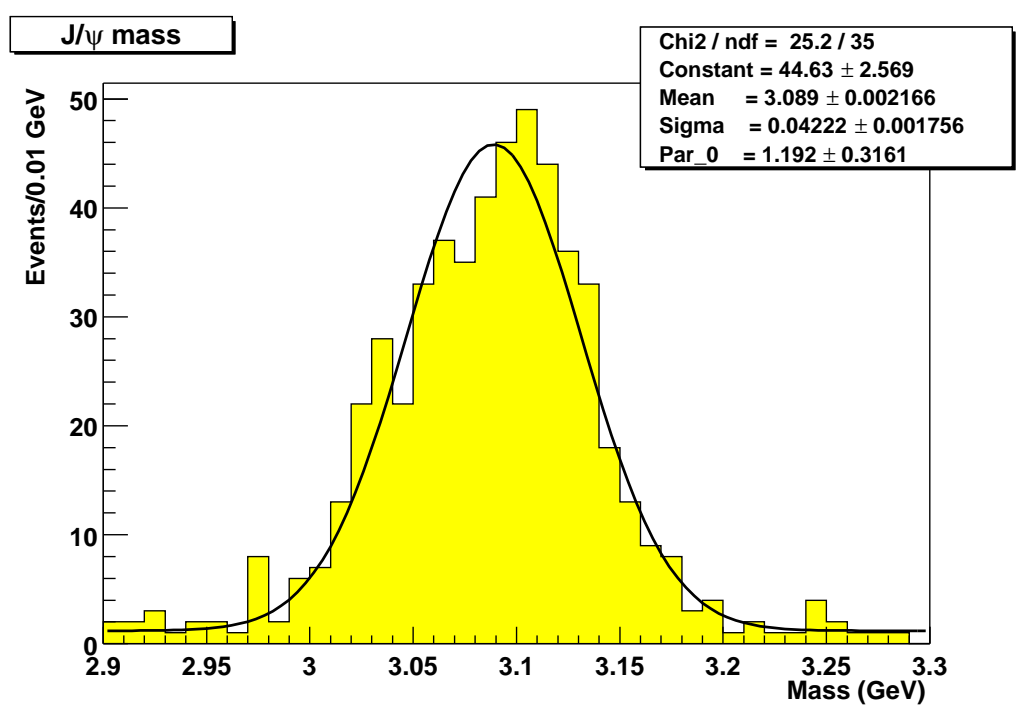

Figure 7.3: Mass of the $J / \psi$ s used to make $B^{+}$'s

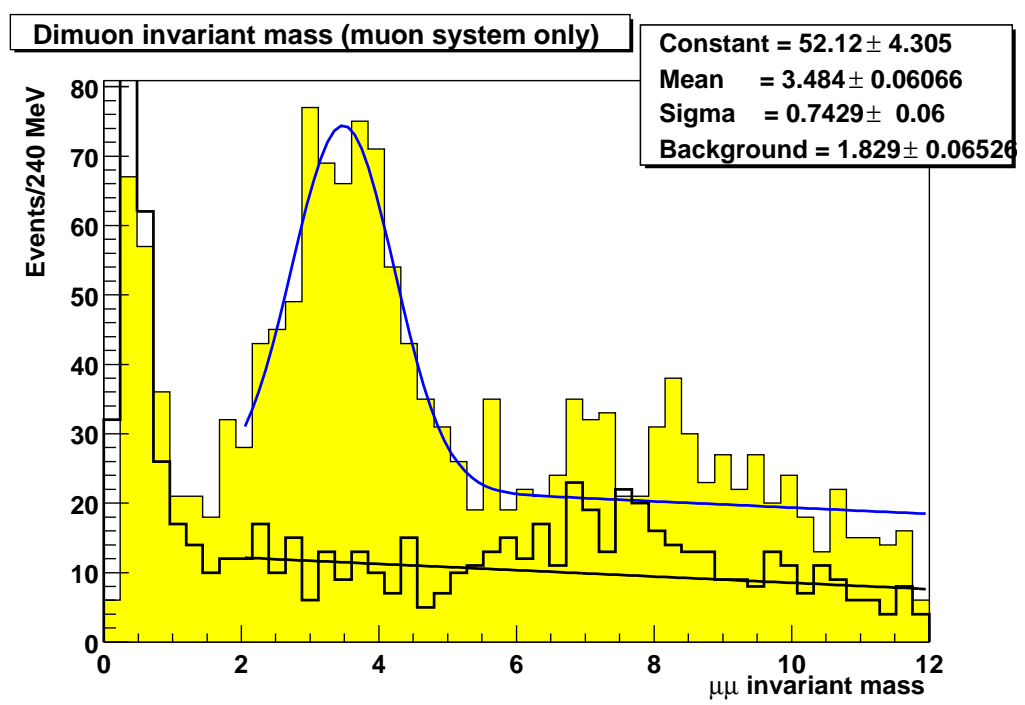

Figure 7.4: $J / \psi$ invariant mass from real data using the muon detectors only. The shaded histogram is the 'opposite charge' dimuon distribution, and the lower histogram is the 'same charge' dimuon distribution. The fit to the 'opposite charge' dimuon distribution is a Gaussian plus a straight line background, the slope of which was obtained by fitting it to the 'same charge' dimuon distribution. 


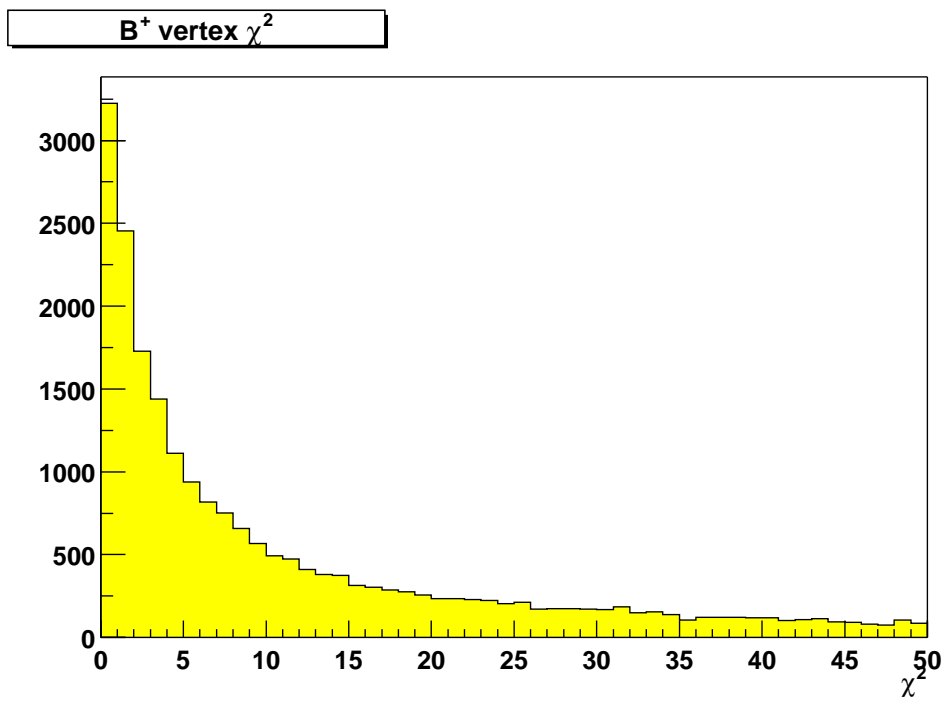

Figure 7.5: $\chi^{2}$ distribution of constrained vertex fit to form $B^{+}$candidates. The three track fit has 3 degrees of freedom. Only the candidate with the largest $\chi^{2}$ probability from each event was used.

Reconstructed $\mathrm{B}^{+}$mass

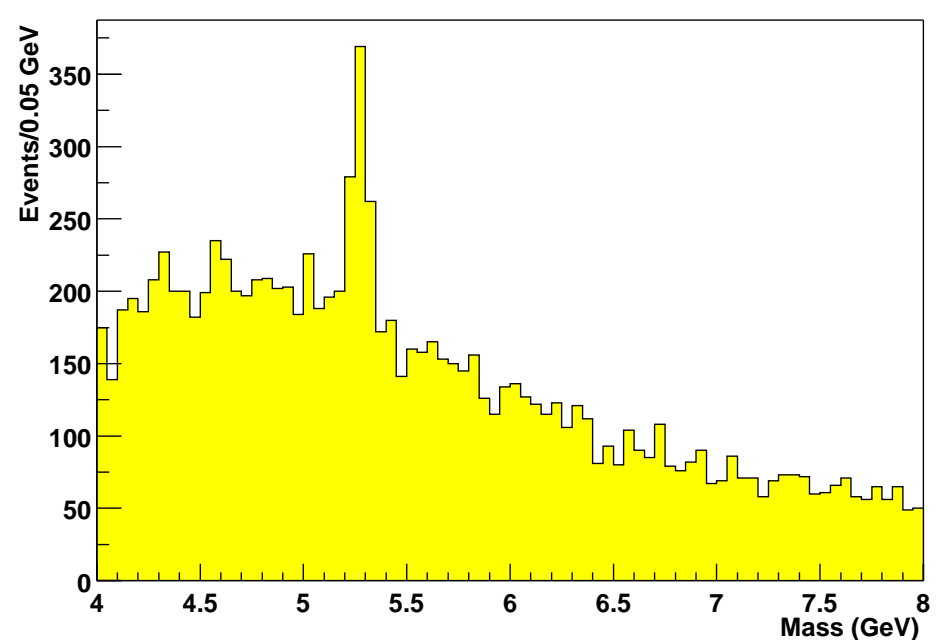

Figure 7.6: Reconstructed invariant mass of all $B^{+}$candidates (Monte Carlo) 


\section{Reconstructed $\mathrm{B}^{+}$mass}

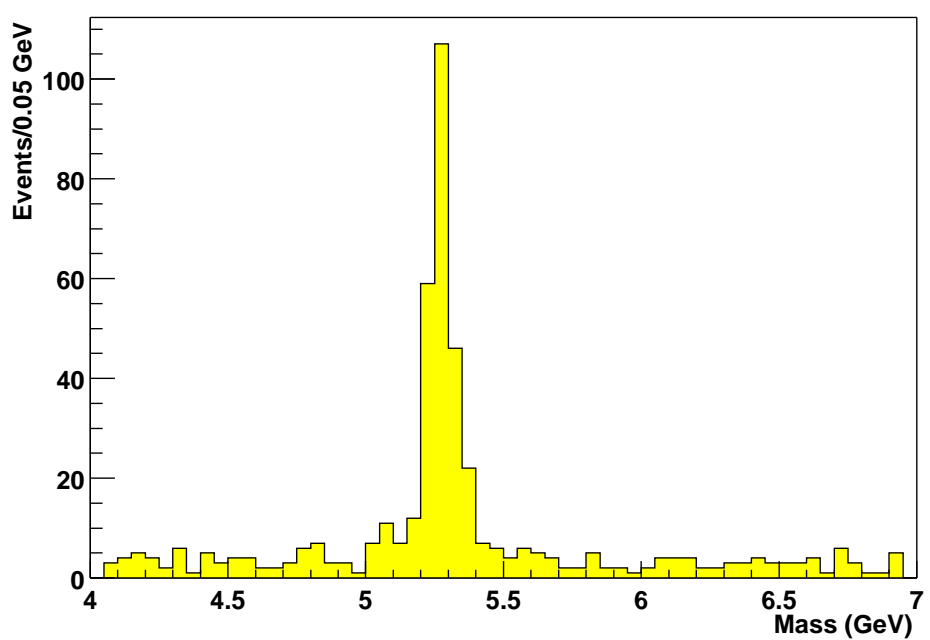

Reconstructed $\mathrm{B}^{+}$mass

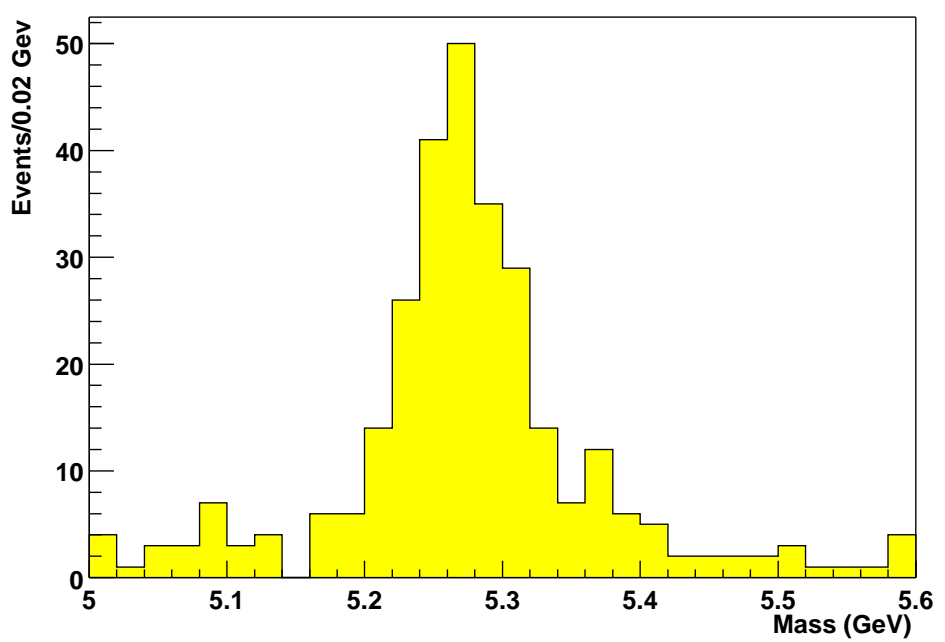

Figure 7.7: Reconstructed $B^{+}$invariant mass using the best candidate from each event. The lower plot shows the peak region in more detail. 
taken in preference to those that have only one. Then the $B^{+}$with the highest $\chi^{2}$ probability for the vertex fit is taken as the 'best' candidate for the event, providing the $\chi^{2}$ probability for the fit is greater than $1 \%$. Figure 7.7 shows the invariant mass distribution of the selected $B^{+}$candidates. Compared with figure 7.6 , almost all the background has been eliminated.

\subsection{Vertexing}

Determining the lifetime of the $B^{+}$mesons requires locating both the secondary vertex at which the $B^{+}$decays, and the primary vertex of the $p \bar{p}$ collision. Finding the $B^{+}$candidates automatically gives the secondary vertex position from the vertex constrained fit, but this still leaves the primary. There are two significant problems in locating the primary vertex in these events:

- Each bunch crossing in the Tevatron has a number of additional interactions on top of the primary interaction (the one that fired the trigger for the event) [1]. These 'minimum-bias' interactions produce tracks which have relatively low transverse momenta. The vertexing algorithm needs to select the primary interaction vertex from among the minimum-bias vertices. Unfortunately $B$ decays produce tracks with relatively low transverse momenta ${ }^{4}$, and the generic vertexing algorithm is not very good at distinguishing $B$ decays from minimum-bias interactions, as it is optimised for high $p_{\mathrm{T}}$ events [87].

- The highest $p_{\mathrm{T}}$ tracks in the event are likely to be the muon tracks from the $J / \psi$ decay, but those tracks are really from the secondary vertex, not the primary. For shorter decay lengths, any vertexing algorithm is likely to associate these tracks with the primary. This may then bias the primary position in the direction of the $B$ decay vertex ${ }^{5}$.

${ }^{4}$ Compared to something like $t \bar{t}$ decays.

${ }^{5}$ There are of course two $B$ mesons in an event, and either or both may bias the vertex position. 
The first point is easily dealt with for these events with fully reconstructed $B^{+}$ mesons. Since the momentum of the $B^{+}$is known it is possible to follow the path of the particle and select the reconstructed vertex it passes closest to. In some cases there may be no vertex near the $B^{+}$path. The likely causes of this are either an incorrectly reconstructed (or totally fake) $B^{+}$or that the primary vertex position has not been reconstructed correctly.

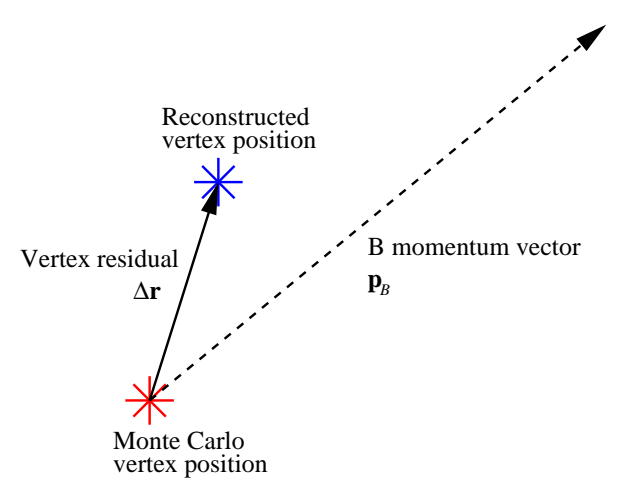

Figure 7.8: Relationship between the actual and reconstructed vertices and the $B^{+}$momentum

The second point is slightly more complicated. To demonstrate the effect, figure 7.9(a) shows the residual primary vertex position projected onto the (Monte Carlo) momentum vector of the $B^{+}$, so (using the terms defined in figure 7.8)

$$
(\text { projected residual })=\Delta r \cdot \hat{\mathbf{p}}_{B}
$$

This clearly shows a bias in the positive direction (towards the $B$ decay vertex) of about $11 \mu \mathrm{m}$. This is not a very large effect (the mean decay length is $\sim 1000 \mu \mathrm{m}$ ), but it will preferentially affect shorter lifetimes. The bias can be reduced by recalculating the primary vertex position, having first removed any of the tracks used to make the $B^{+}$that were also used in the primary vertex fit. The results are shown in figure 7.9(b), in which the bias is reduced to $\sim 3 \mu \mathrm{m}$.

With the recalculation of the primary vertex position done it is then possible to determine the resolution of the vertex position. Figure 7.10 shows the residuals for 


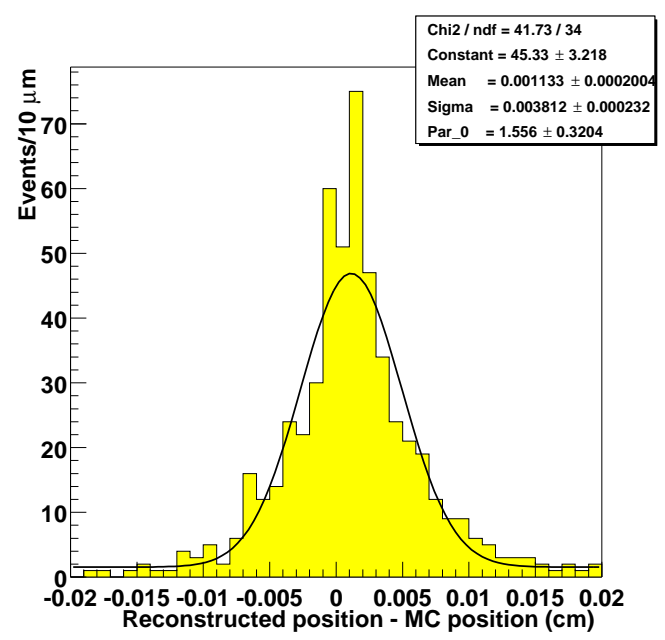

(a) Residual projected onto the Monte Carlo $B^{+}$momentum vector, using the initial primary vertex position.

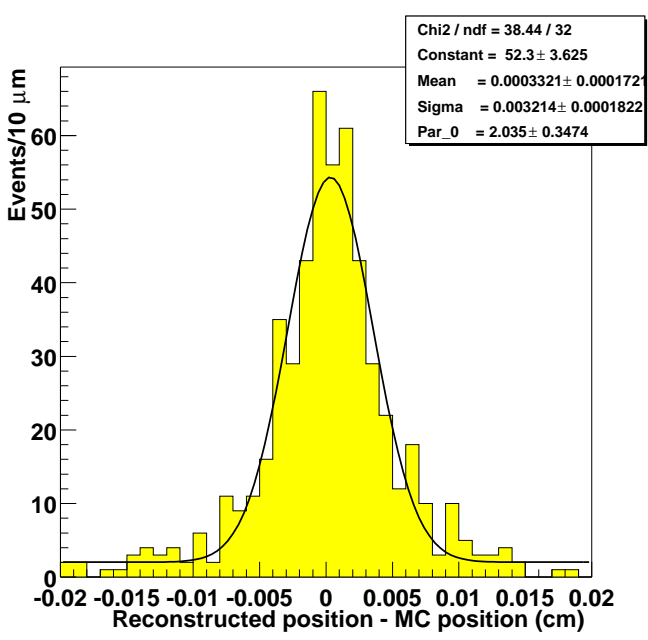

(b) Residual projected onto the Monte Carlo $B^{+}$momentum vector, using the recalculated primary vertex position.

Figure 7.9: Bias on primary vertex position

the $x$ and $z$ coordinates $^{6}$. The resolution of the primary vertex is therefore $25 \mu \mathrm{m}$ in the $x y$ plane, and $40 \mu \mathrm{m}$ in $z$.

Figure 7.11 shows the resolution of the secondary vertex position, which is just the position given by the vertex constrained fit for the $B^{+}$. Here the $x y$ resolution is $23 \mu \mathrm{m}$ and the $z$ resolution is $45 \mu \mathrm{m}$. The reason why the secondary vertex resolution, with only three tracks, is as good as the primary vertex resolution, with a larger number of tracks, is that the secondary vertex contains the highest transverse momentum tracks (the muons), while the primary is left with the low transverse momentum tracks.

\footnotetext{
${ }^{6}$ The $y$ coordinate plot is very similar to the one for $x$ and has been omitted.
} 

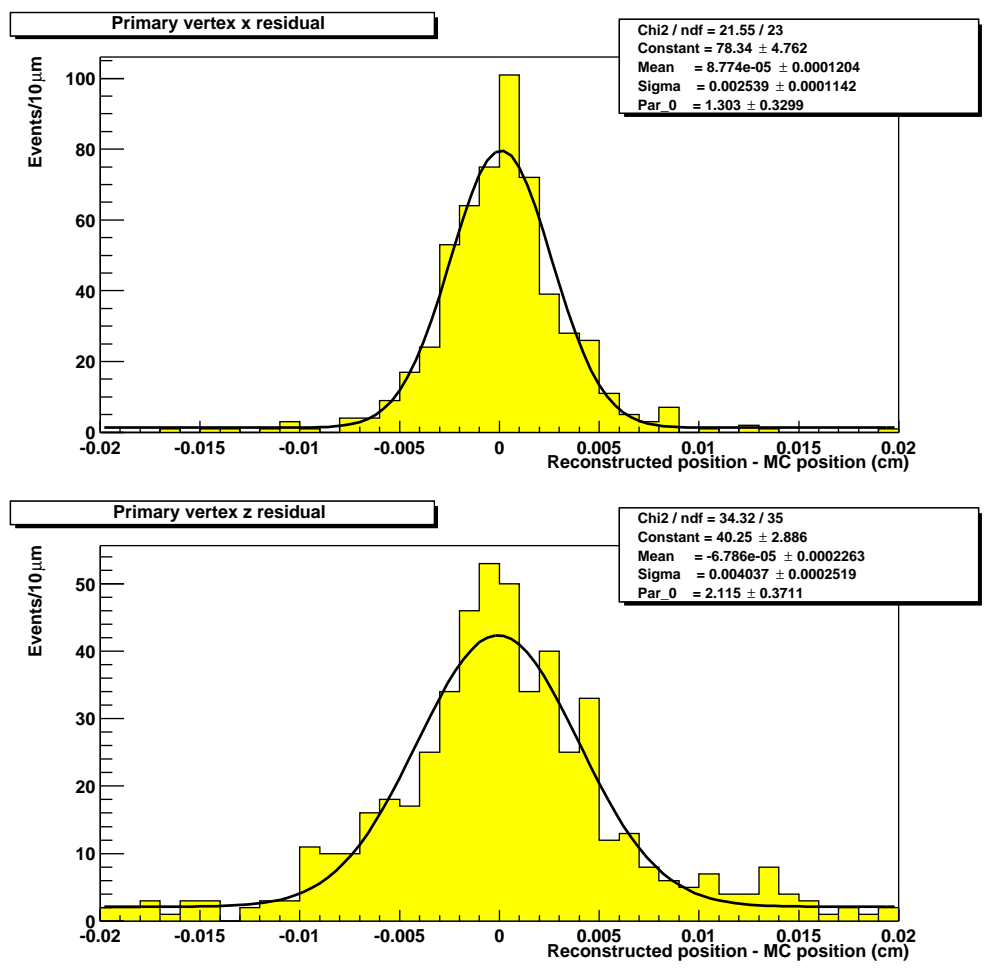

Figure 7.10: Primary vertex resolution

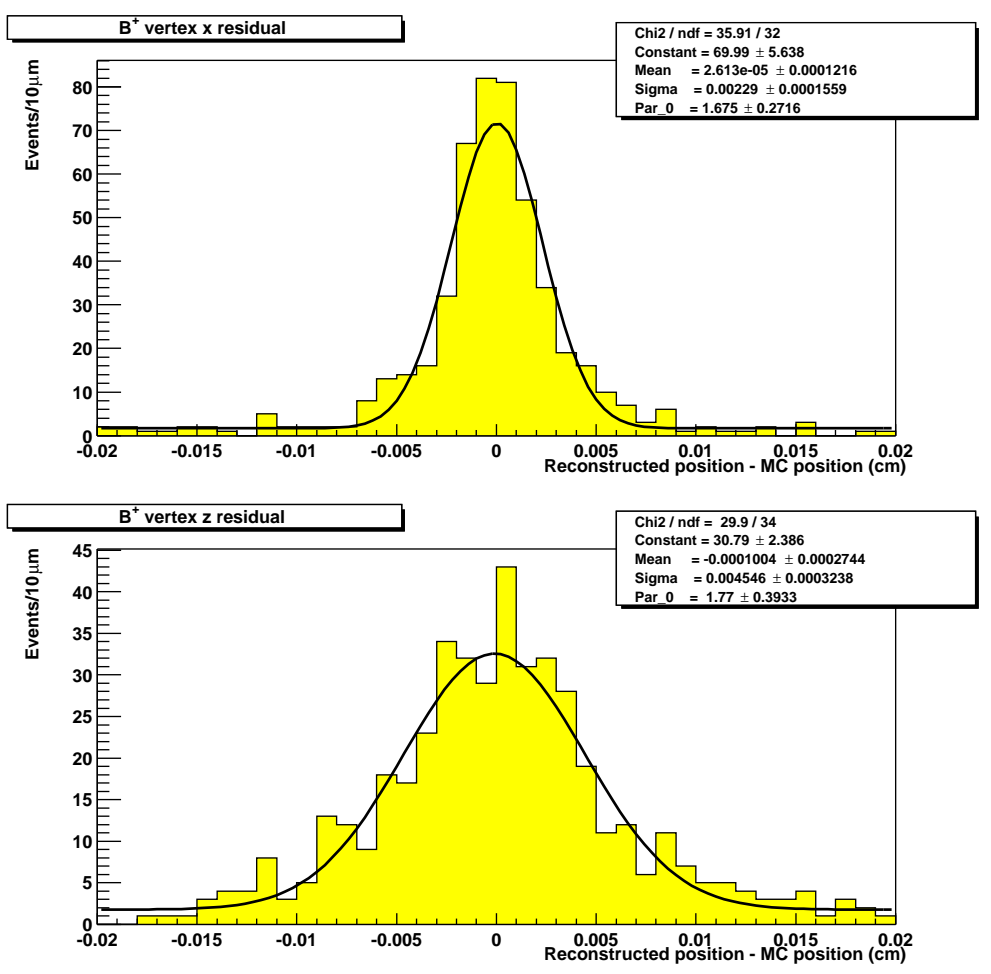

Figure 7.11: Secondary vertex resolution 


\subsection{Lifetime}

The decay length vector for the $B^{+}$is given by the difference between the secondary and primary vertex positions

$$
\mathbf{d}=\mathbf{x}_{\text {secondary }}-\mathbf{x}_{\text {primary }}
$$

The decay length in the detector frame is related to the proper decay length by ${ }^{7}$

$$
\mathbf{d}=\boldsymbol{\beta} \gamma L
$$

where $\boldsymbol{\beta}$ is the velocity of the $B, \gamma$ is the Lorentz boost factor $\gamma=1 / \sqrt{1-\beta^{2}}$, and $L$ is the proper decay length.

The momentum of the $B$ is related to its velocity by

$$
\mathbf{p}=\boldsymbol{\beta} \gamma M_{B}
$$

with $M_{B}$ as the mass of the $B^{+}$. Together with equation (7.3), this gives

$$
\mathbf{d}=\frac{\mathbf{p}}{M_{B}} L
$$

Equation (7.5) requires that the momentum and decay length vectors are proportional to one another. As the $B$ momentum and decay length are separately measured, even for a well reconstructed decay the errors on both quantities mean that they will not be exactly proportional. For an incorrectly reconstructed $B$, the momentum and decay vectors may even point in opposite directions. Some method is needed to combine the two measurements (and their errors) to give a value for the proper lifetime. One way of proceeding is to take the decay length to be the

${ }^{7}$ With the speed of light $c=1$ there is effectively no distinction between the decay length and the decay time. 
projection of the decay length vector on to the $B$ momentum vector, $\mathbf{d} \cdot \hat{\mathbf{p}}$. For a well constructed $B$ this quantity is almost identical to $|\mathbf{d}|$. However, for a badly reconstructed $B$, it can differ significantly, and for the case where the momentum is opposite to the decay vector it becomes negative. For mis-reconstructed $B$ s which really come from prompt (i.e. very short lifetime) decays, where the momentum and decay vectors are more-or-less uncorrelated, the proper lifetime will be distributed symmetrically around zero, which makes it easy to fit a suitable function in order to parametise the background distribution (see section 7.8).

Using this method the estimated proper decay length is given by

$$
L=\frac{\mathbf{d} \cdot \mathbf{p}}{p^{2}} M_{B}
$$

The error on the proper lifetime can be calculated by differentiating equation (7.6):

$$
\sigma(L)^{2}=\frac{M_{B}^{2}}{p^{4}}\left[\mathbf{p}^{T} V_{\mathbf{d}} \mathbf{p}+\left(\mathbf{d}-\frac{2(\mathbf{d} \cdot \mathbf{p})}{p^{2}} \mathbf{p}\right)^{T} V_{\mathbf{p}}\left(\mathbf{d}-\frac{2(\mathbf{d} \cdot \mathbf{p})}{p^{2}} \mathbf{p}\right)\right]+\frac{(\mathbf{d} \cdot \mathbf{p})^{2}}{p^{4}} \sigma\left(M_{B}\right)^{2}
$$

where $V_{\mathbf{d}}$ and $V_{\mathbf{p}}$ are the covariance matrices of $\mathbf{d}$ and $\mathbf{p}$, and $\sigma\left(M_{B}\right)$ is the error on the $B^{+}$mass. The masses of the $B$ mesons are known to an accuracy of $\sim 0.05 \%$ [12], and so this component of the error is negligible.

As well as the full three dimensional proper decay length given by equation (7.6) it is possible to calculate the decay length using only the transverse components. From equation (7.5)

$$
d_{x}=\frac{p_{x}}{M_{B}} L \quad \text { and } \quad d_{y}=\frac{p_{y}}{M_{B}} L
$$

SO

$$
p_{x} d_{x}=\frac{p_{x}^{2}}{M_{B}} L \quad \text { and } \quad p_{y} d_{y}=\frac{p_{y}^{2}}{M_{B}} L
$$


and therefore if $\mathbf{d}_{T}$ is the vector between the primary and secondary vertices in the $x y$ plane only, and $\mathbf{p}_{T}$ the corresponding $B$ momentum (and so $\mathbf{d}_{T} \cdot \mathbf{p}_{T}=p_{x} d_{x}+p_{y} d_{y}$ ), the proper decay length is given by

$$
L=\frac{\mathbf{d}_{T} \cdot \mathbf{p}_{T}}{p_{T}^{2}} M_{B}
$$

\subsection{Background sample}

A real $B^{+}$sample will contain background events from non- $B^{+}$decays. An accurate estimate of the relative proportions of signal and the various background components requires the use of the $D \varnothing$ trigger simulator, which was not available at the time of writing. However, some preliminary studies have been done [88, chapter 4]. The primary source of background is events with real $J / \psi$ particles used to make fake $B^{+}$s. To simulate this, two separate background samples were used $-25,000$ direct $J / \psi$ production $(q \bar{q} \rightarrow J / \psi+X)$ events, and 27,000 inclusive $b \bar{b} \rightarrow J / \psi$ $(q \bar{q} \rightarrow b \bar{b} \rightarrow J / \psi+X)$ events $^{8}$, both created with no generator level $p_{T}$ cuts. As noted in the reference above there is a great degree of uncertainty in the relative proportions of the background components, but these are compatible with the figures given there. The signal plus background $B^{+}$mass distribution is shown in figure 7.12, and the lifetime distribution in figure 7.13. As expected, the direct $J / \psi$ component is largely at short lifetimes.

\subsection{Lifetime fits}

\subsubsection{Two dimensional lifetime distribution}

The distribution of the reconstructed $B^{+}$lifetimes using the two dimensional proper lifetime from equation (7.8) is shown in figure 7.14. The difference between the

\footnotetext{
${ }^{8}$ As an inclusive sample, this will include more $B^{+} \rightarrow J / \psi K^{+}$events as well as background
} 


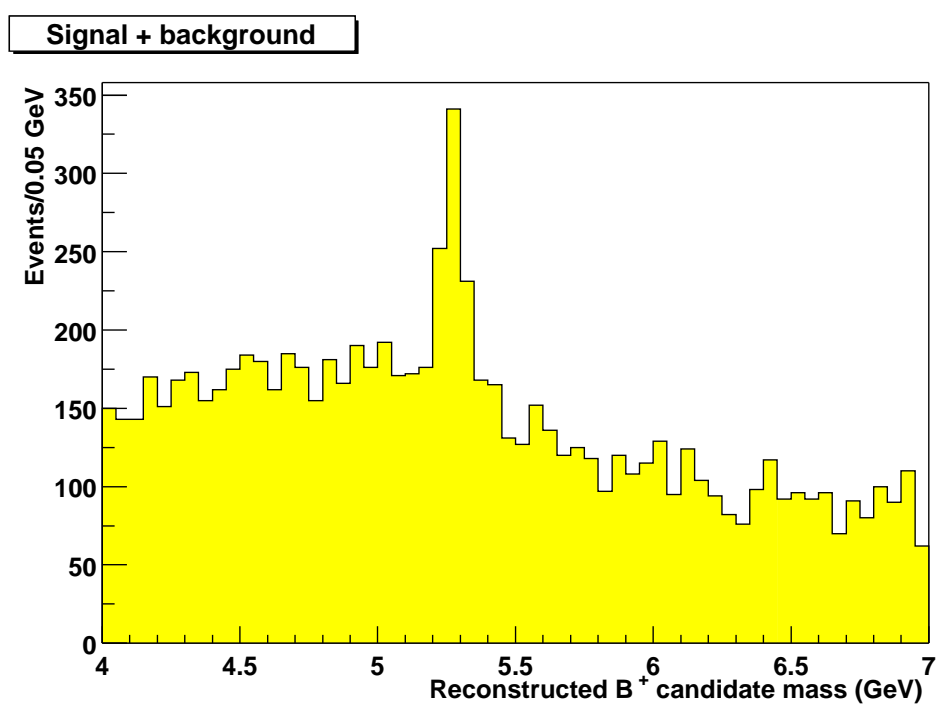

Figure 7.12: Reconstructed $B^{+}$mass from combined signal and background samples

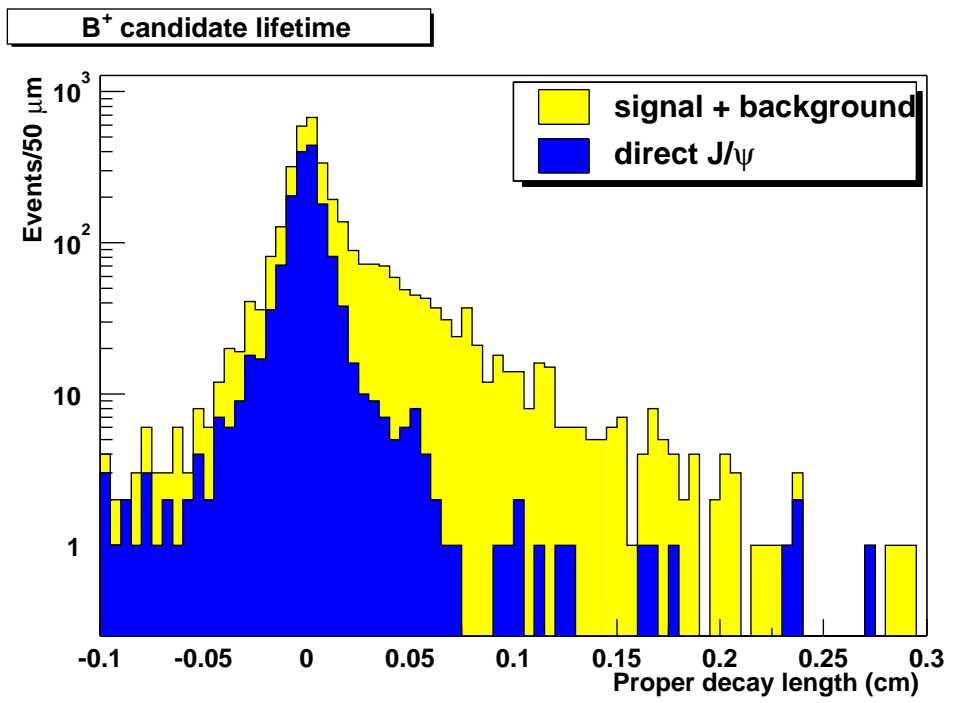

Figure 7.13: Reconstructed $B^{+}$proper decay length (in a $1 \mathrm{GeV}$ window around the $B^{+}$mass) from the combined signal and background, and from direct $J / \psi$ production alone 
reconstructed proper decay length and the Monte Carlo decay length is shown in figure 7.15. This distribution has a more significant tail on the negative side - this is reasonable as fake $B^{+}$particles are more likely to be formed near the primary decay vertex due to the number of tracks there, making the decay length shorter than it should be. Figure 7.16 shows the mean error on the proper decay length plotted against the proper decay length. Finally, figure 7.17 shows the difference between the reconstructed and Monte Carlo proper lifetimes divided by the error on the lifetime. As expected the peak of this distribution is a Gaussian with $\sigma \sim 1$, showing that the calculated error values are reasonable.

\subsubsection{Three dimensional lifetime distribution}

The three dimensional lifetime takes advantage of the extra information available from the $z$ coordinate resolution of the silicon detector. Previous measurements from CDF of the lifetime from fully reconstructed $B$ mesons [83] only used the transverse decay length as CDF's then vertex detector gave no precision z position information. The $90^{\circ}$ stereo angle strips in the new D $\varnothing$ vertex detector give an accurate measurement of the $z$ position of the primary and secondary vertices (from section 7.5 the $z$ vertex resolutions for primary and secondary vertices are $\sim 40 \mu \mathrm{m}$ ), and so it is possible to use the full three dimensional decay length. Figures 7.18, $7.19,7.20$, and 7.21 show similar plots as for the last section, but for the three dimensional proper lifetime.

\subsubsection{Likelihood fit to lifetime}

Having obtained the reconstructed proper lifetime distributions (figures 7.14 and 7.18) the mean lifetime can then be recovered by fitting a suitable function to the data. The method chosen was to perform an unbinned extended maximum likelihood fit. A brief description of the maximum likelihood method, along with various probability density functions, is given in Appendix A. 


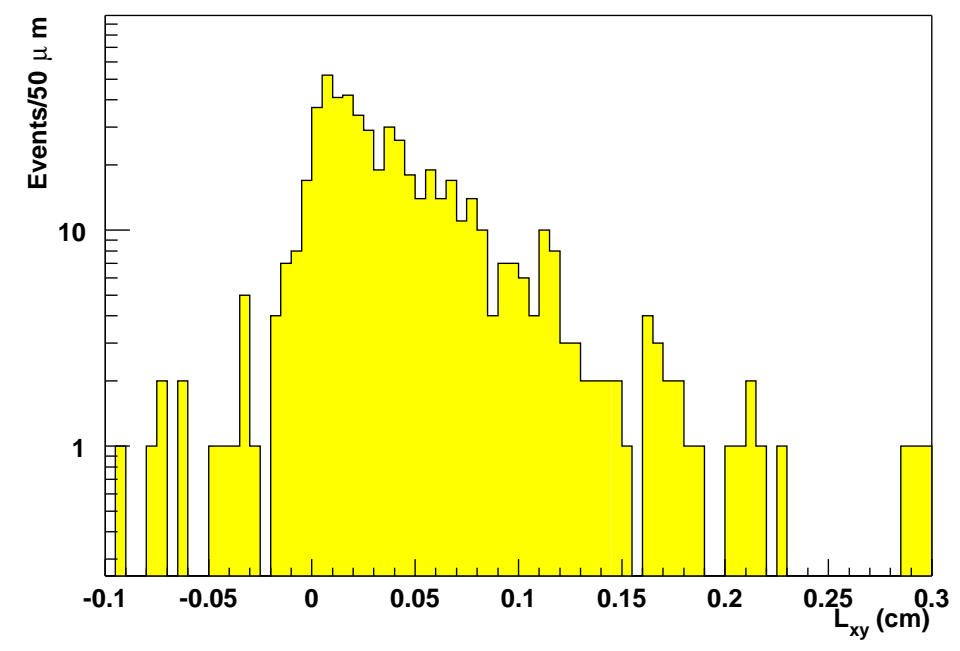

Figure 7.14: $2 \mathrm{D}$ proper decay length for $B^{+}\left(B^{+} \rightarrow J / \psi K^{+}\right.$events only without background included)
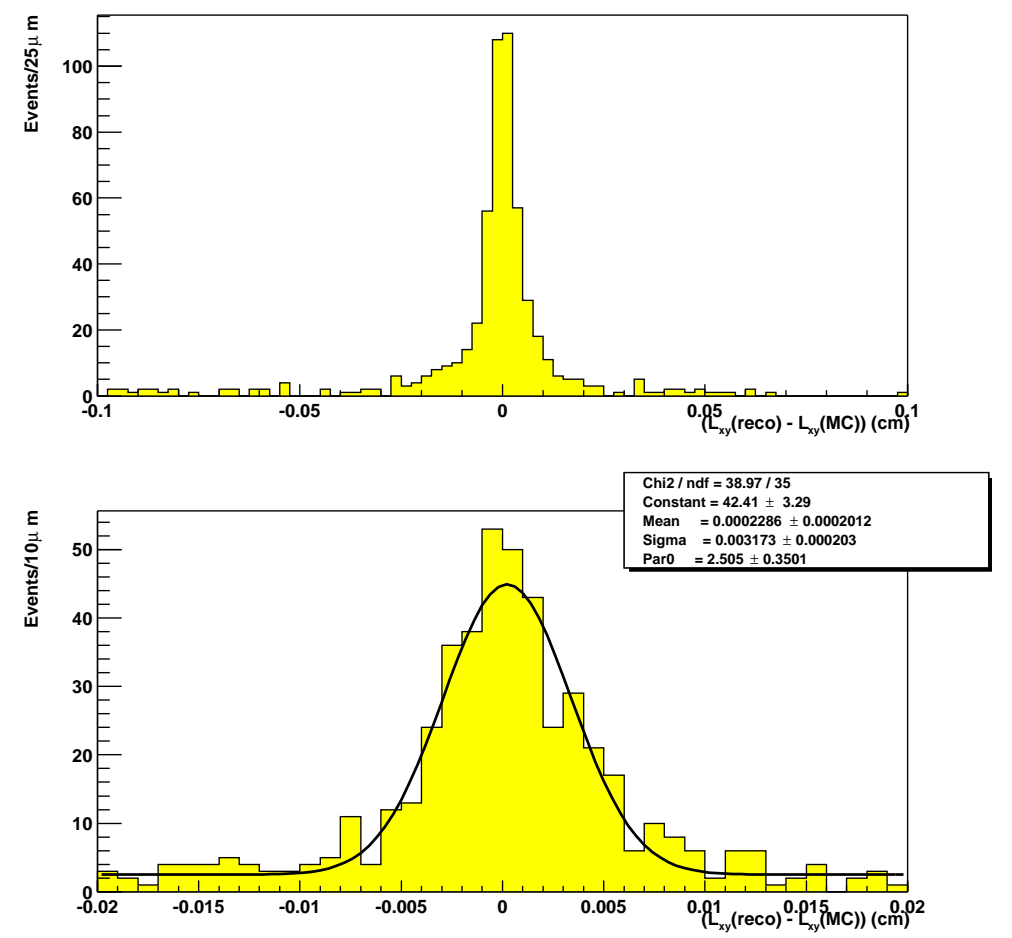

Figure 7.15: Difference between reconstructed $B^{+} 2 \mathrm{D}$ proper decay length and Monte Carlo proper decay length. The lower plot shows the peak region in more detail. 


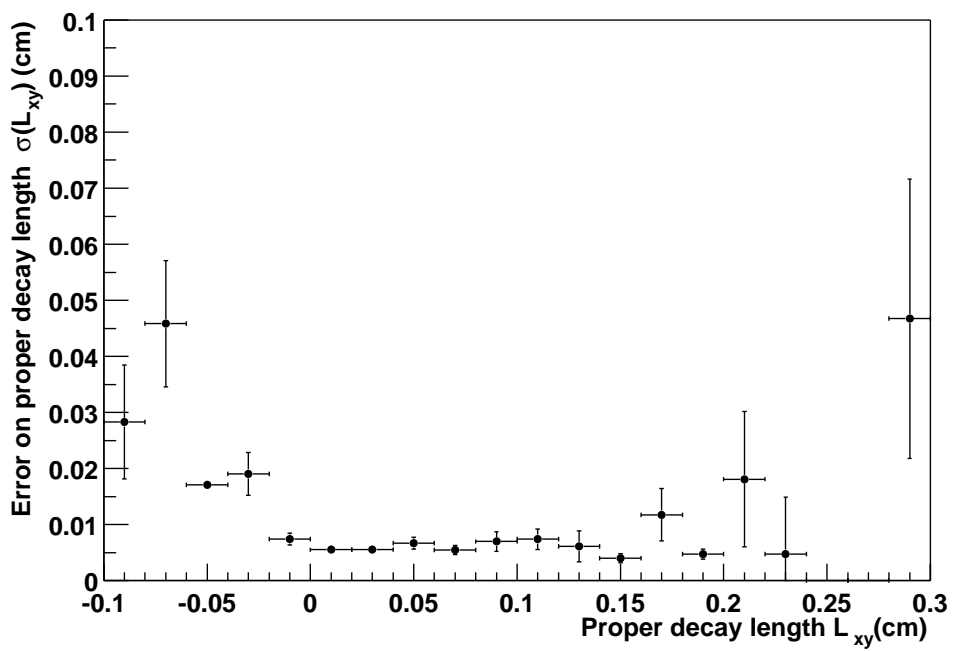

Figure 7.16: The errors on the 2D proper decay length

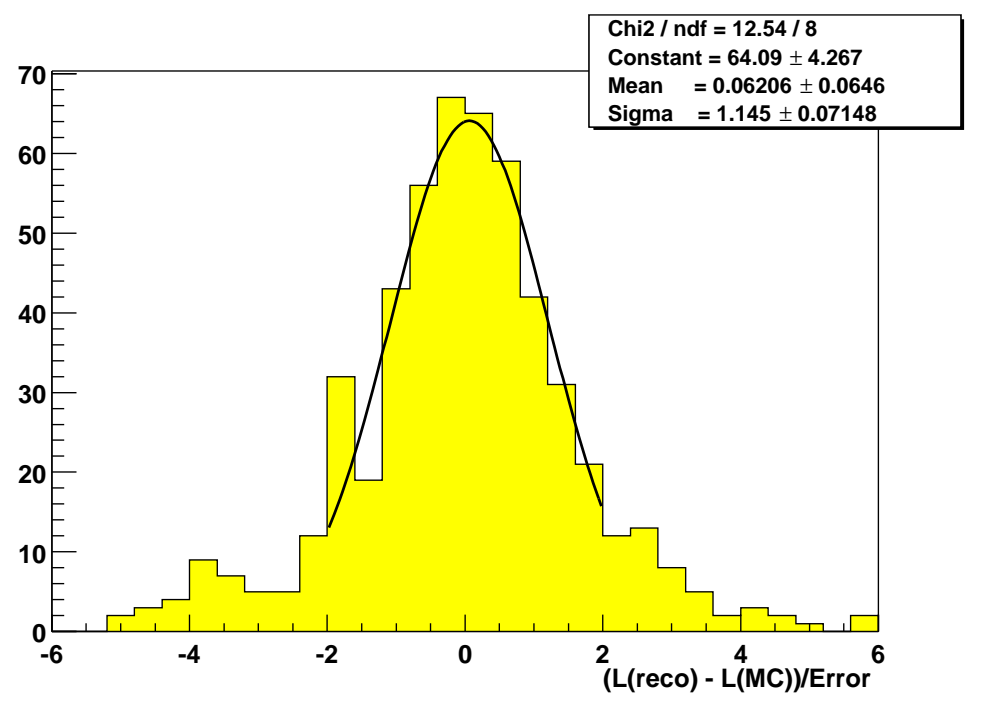

Figure 7.17: Difference between reconstructed $B^{+} 2 \mathrm{D}$ proper decay length and Monte Carlo proper decay length, divided by the error on the reconstructed length. 


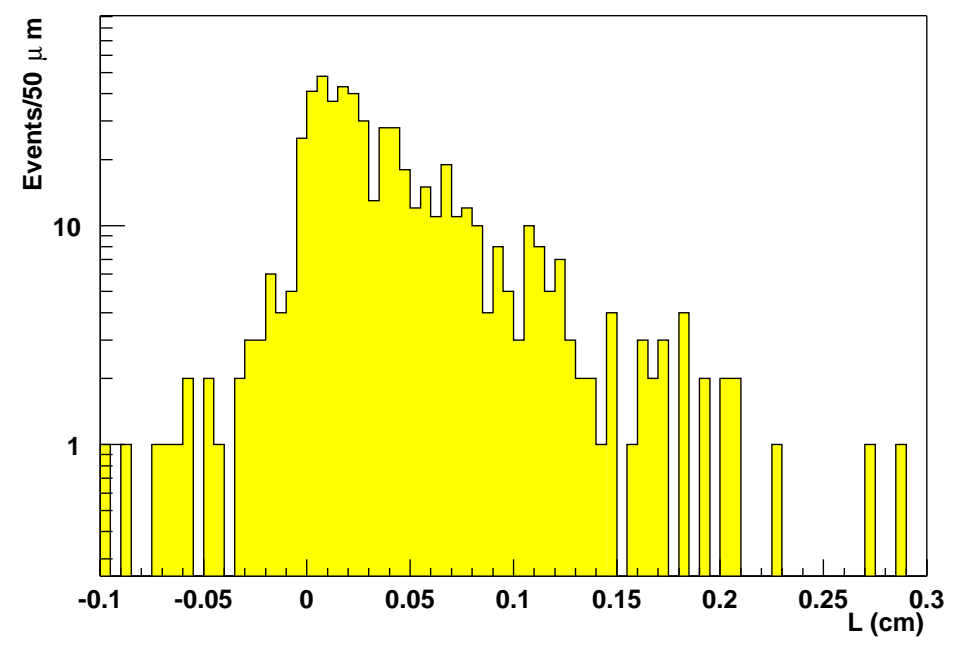

Figure 7.18: 3D proper decay length for $B^{+}\left(B^{+} \rightarrow J / \psi K^{+}\right.$events only $)$
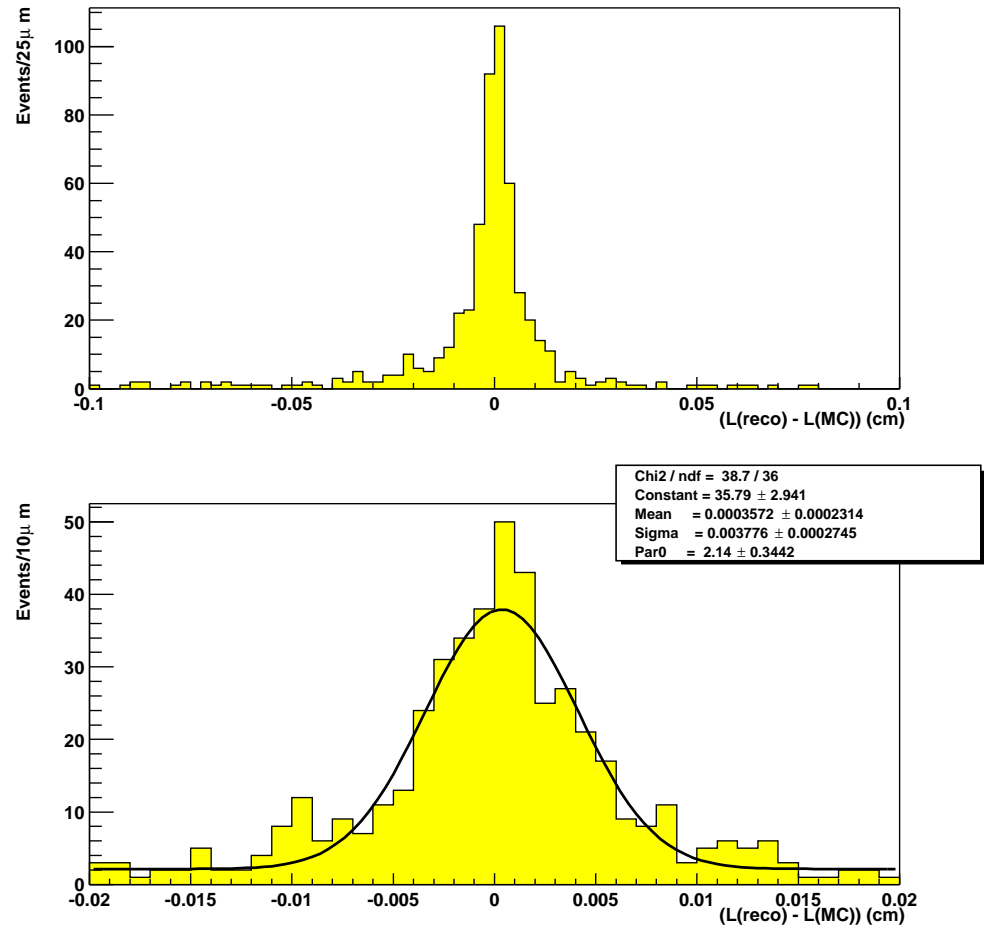

Figure 7.19: Difference between reconstructed $B^{+} 3 \mathrm{D}$ proper decay length and Monte Carlo proper decay length. The lower plot shows the peak region in more detail. 


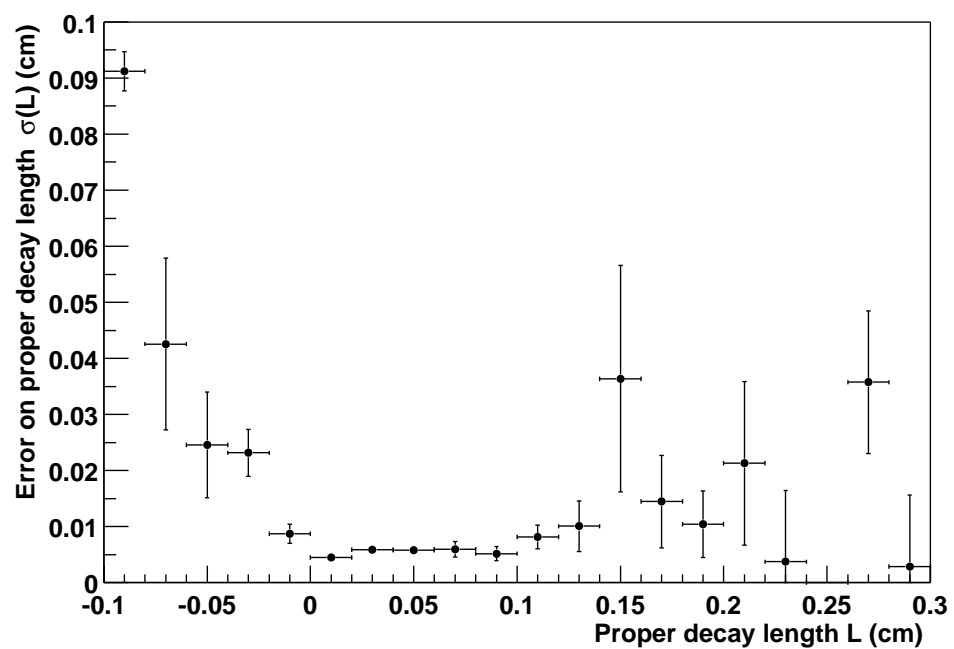

Figure 7.20: The errors on the 3D proper decay length

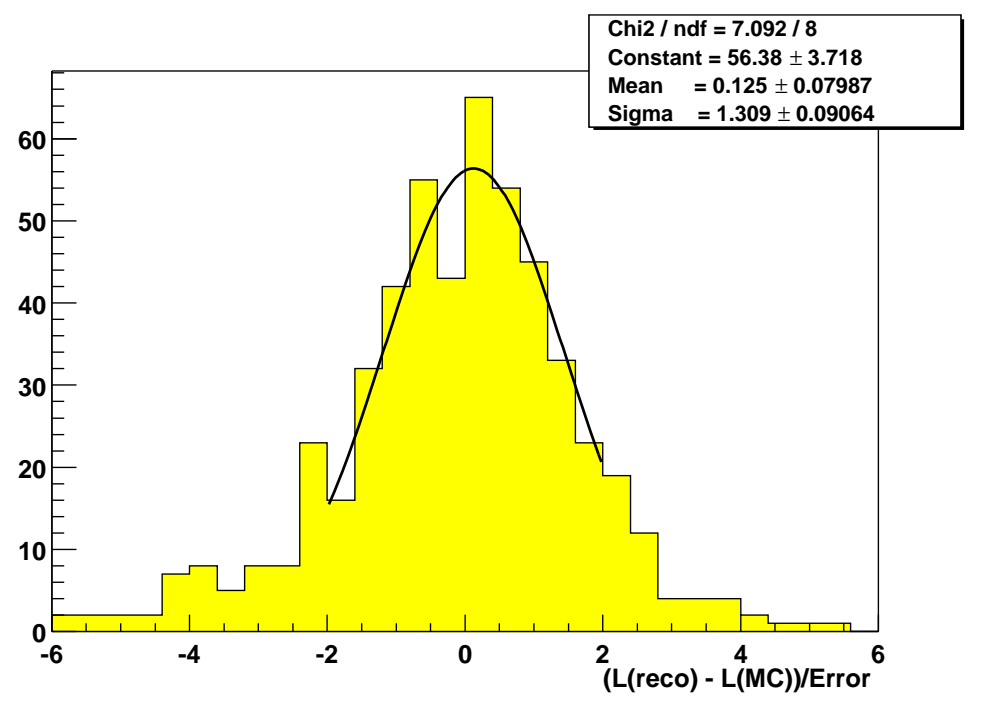

Figure 7.21: Difference between reconstructed $B^{+}$proper decay length and Monte Carlo 3D proper decay length, divided by the error on the reconstructed length. 
The probability density function for the lifetime of a particle is an exponential decay as given by equation (A.8). However, the effect of the measurement error on the lifetime is to make an exponential decay, convoluted with a Gaussian resolution function, as given by equation (A.10), more suitable.

The background is assumed to be a combination of a Gaussian peak with nonGaussian tails represented by positive and negative exponential functions. Because some of the positive exponential tail represents the decay of actual longlived particles (for example, a $B^{+}$reconstructed with an incorrect $K^{+}$track, and so having the wrong proper lifetime as the momentum will be wrong), the fraction of the background in each tail can be different.

The parameters that were used in the fitting are summarised in table 7.1. The likelihood function for each event using these parameters is

$$
\begin{aligned}
\mathcal{L}_{i}= & N_{\mathrm{sig}} \mathcal{F}\left(L_{i}, \sigma_{i} \mid \lambda\right) \\
+ & N_{\mathrm{bkg}} \mathcal{L}_{\mathrm{bkg}}
\end{aligned}
$$

where the likelihood of the background distribution is given by

$$
\begin{aligned}
\mathcal{L}_{\mathrm{bkg}}= & \left(1-f_{+}-f_{-}\right) \mathcal{G}\left(L_{i}, \sigma_{i}\right) \\
& + \begin{cases}\frac{f_{+}}{\lambda_{+}} e^{-L_{i} / \lambda_{+}} & \text {if } L_{i} \geq 0, \\
\frac{f_{-}}{\lambda_{-}} e^{L_{i} / \lambda_{-}} & \text {if } L_{i}<0 .\end{cases}
\end{aligned}
$$

\begin{tabular}{|c|l|}
\hline Parameter & Meaning \\
\hline$\lambda$ & $B$ lifetime \\
$N_{\text {sig }}$ & Number of signal events \\
$f_{+}$ & Fraction of background events in positive tail \\
$\lambda_{+}$ & Lifetime of positive tail \\
$f_{-}$ & Fraction of background events in negative tail \\
$\lambda_{-}$ & Lifetime of negative tail \\
$N_{\text {bkg }}$ & Number of background events in signal sample \\
$N_{\text {sideband }}$ & Number of events in sideband sample \\
\hline
\end{tabular}

Table 7.1: Parameters used in proper lifetime fits 
where $\mathcal{F}$ and $\mathcal{G}$ are respectively the probability density functions for an exponential decay convoluted with a Gaussian resolution function, and for a Gaussian distribution, as defined in section A.3. The likelihood fit is then given by maximising the function

$$
\sum \log \mathcal{L}_{i}-N_{\text {sig }}-N_{\mathrm{bkg}}
$$

where the sum is over all the calculated proper lifetimes and errors $\left(L_{i}, \sigma_{i}\right)$.

The form of the background can be found by fitting the background part of the likelihood function to the sidebands of the reconstructed $B^{+}$sample. The extended likelihood function ${ }^{9}$ for this is given by

$$
\sum \log \left(N_{\text {sideband }} \mathcal{L}_{\text {bkg }}\right)-N_{\text {sideband }}
$$

The fit was performed by simultaneously fitting the background likelihood function (7.12) to the sideband data sample, defined as events with a reconstructed mass difference from the PDG $B^{+}$mass of $0.2<\Delta M<0.3$, and the full likelihood function (7.11) to the signal region $\Delta M<0.2$.

The results of performing this fit on the transverse proper decay length are shown in table 7.2 and are plotted in figure 7.22. Similarly, the results for the three dimensional lifetime are shown in table 7.3 and figure 7.23 .

The mean $B^{+}$lifetime used in the Monte Carlo simulation was $460 \mu \mathrm{m}$ (1.53 ps). For both the two dimensional and three dimensional cases the fitted mean lifetime (parameter $\lambda$ in the fit) is reasonably close $(\sim 1 \sigma)$ to the simulated value. The errors in both cases are very similar - the three dimensional decay length is scaled by a smaller factor than the two dimensional decay length to obtain the proper length, but this is offset by the larger errors on the $z$ component of the length. However,

\footnotetext{
${ }^{9}$ There is no major advantage in using the extended likelihood for this part of the fit, as it only contains one component. However, it is easier to implement the two parts (signal \& sidebands) in an equivalent manner.
} 


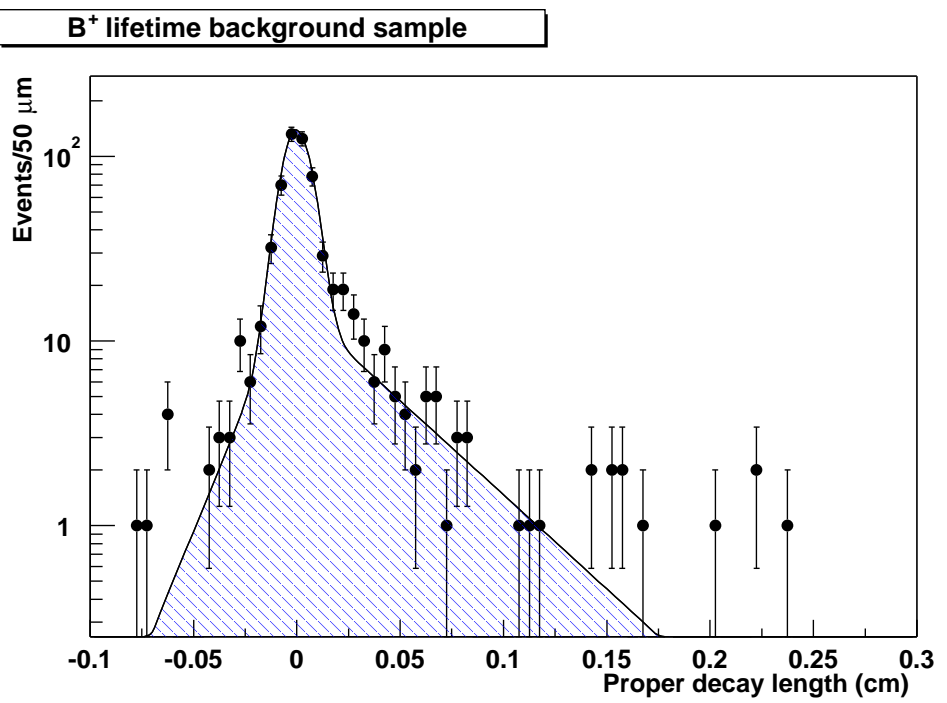

(a) Fit to transverse proper decay length sideband

\section{$\mathrm{B}^{+}$lifetime}

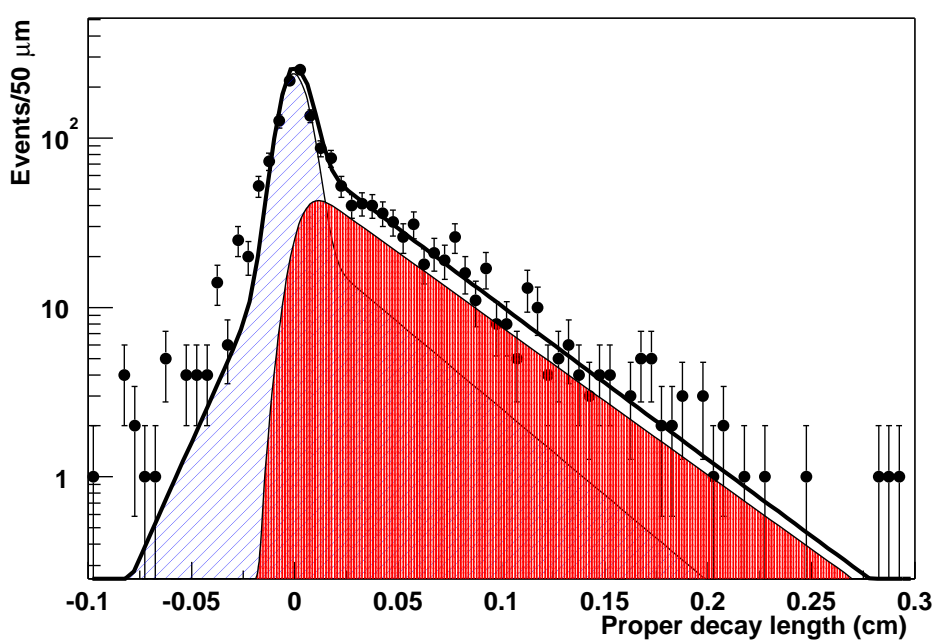

(b) Fit to transverse proper decay length. The hatched area is the background contribution, the shaded area is the $B^{+}$lifetime component, and the solid line is the combined distribution.

Figure 7.22: Background and signal fits to the transverse proper decay length. In both cases the fit function is plotted with a fixed value of the resolution, but in the fitting procedure the error on each separate data point is used. 
$\mathrm{B}^{+}$lifetime background sample

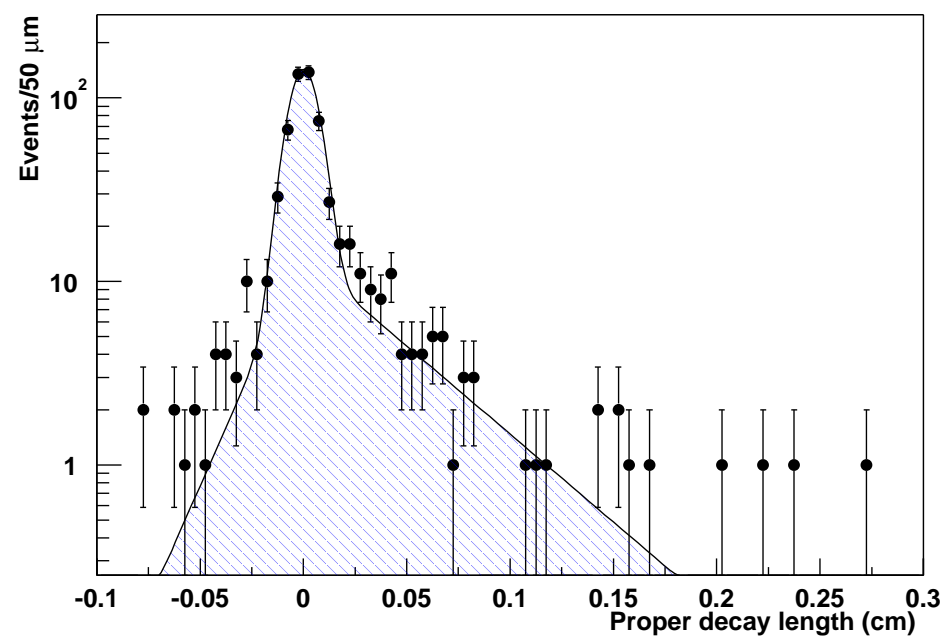

(a) Fit to three dimensional proper decay length sideband

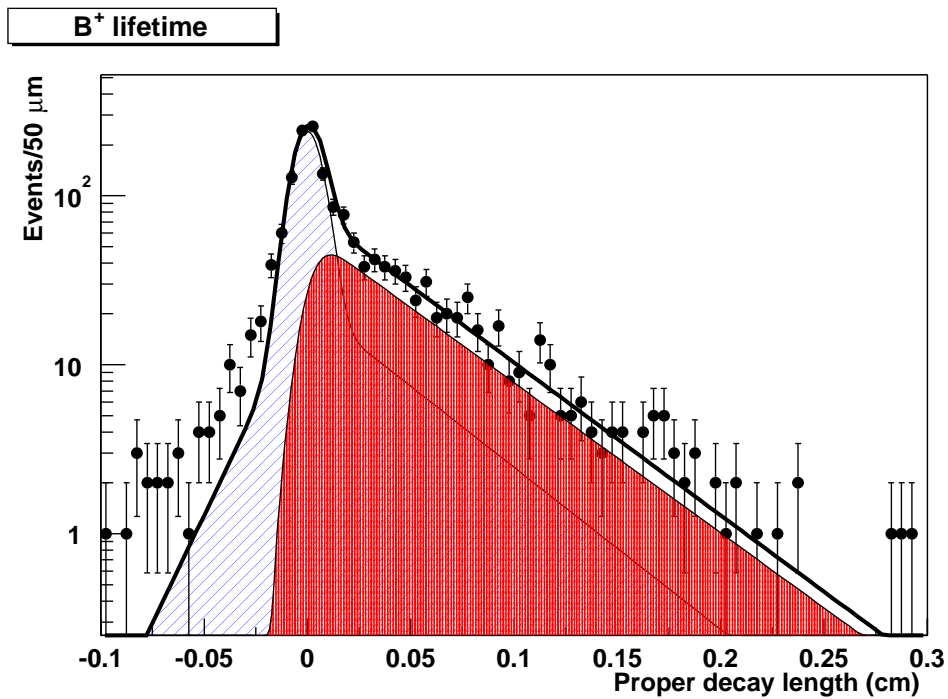

(b) Fit to three dimensional proper decay length. The hatched area is the background contribution, the shaded area is the $B^{+}$ lifetime component, and the solid line is the combined distribution.

Figure 7.23: Background and signal fits to the three dimensional proper decay length. In both cases the fit function is plotted with a fixed value of the resolution, but in the fitting procedure the error on each separate data point is used. 


\begin{tabular}{|c|c|}
\hline Parameter & Fit value \\
\hline$\lambda$ & $496.0 \pm 32.3 \mu \mathrm{m}$ \\
$N_{\text {sig }}$ & $566 \pm 43$ \\
$f_{+}$ & $0.21 \pm 0.02$ \\
$\lambda_{+}$ & $426.2 \pm 42.9 \mu \mathrm{m}$ \\
$f_{-}$ & $0.11 \pm 0.02$ \\
$\lambda_{-}$ & $159.5 \pm 18.4 \mu \mathrm{m}$ \\
$N_{\text {bkg }}$ & $1077 \pm 45$ \\
$N_{\text {sideband }}$ & $627 \pm 25$ \\
\hline
\end{tabular}

Table 7.2: Results of likelihood fit to transverse proper decay length

\begin{tabular}{|c|c|}
\hline Parameter & Fit value \\
\hline$\lambda$ & $488.9 \pm 31.0 \mu \mathrm{m}$ \\
$N_{\text {sig }}$ & $584 \pm 42$ \\
$f_{+}$ & $0.19 \pm 0.02$ \\
$\lambda_{+}$ & $453 \pm 46.7 \mu \mathrm{m}$ \\
$f_{-}$ & $0.08 \pm 0.01$ \\
$\lambda_{-}$ & $170.6 \pm 25.3 \mu \mathrm{m}$ \\
$N_{\text {bkg }}$ & $1052 \pm 46$ \\
$N_{\text {sideband }}$ & $627 \pm 24$ \\
\hline
\end{tabular}

Table 7.3: Results of likelihood fit to three dimensional proper decay length 
even if it does not improve the fit parameters, using the $z$ component can help to reject badly or incorrectly reconstructed $B$ mesons where the problems are not visible using just the $x y$ plane information.

As stated in section 7.7 there is some uncertainty over the correct ratio of signal to background, and the relative distributions of the different background components. However, the lifetime fit is not particularly susceptible to the amount of background present (doing the reconstruction and fit without any background yields a very similar error on the lifetime), so the lifetime resolution should not be greatly affected by differences in the makeup of the background components.

An error value of $31 \mu \mathrm{m}$ corresponds to an error of 0.10 ps on the lifetime (compare with the world average error of 0.027 ps given on page 35). However, the results are limited by the size of the Monte Carlo data sample. Based on the numbers given in references [83] and [88, chapter 8] DØ can expect to obtain of the order of 12000 events in the $B^{+} \rightarrow J / \psi K^{+}$decay channel with $2 \mathrm{fb}^{-1}$ of integrated luminosity, which would reduce the purely statistical error to $\sim 0.02$ ps. The expected number of reconstructed $B^{+}$in all channels is $\sim 40000$, which would reduce the error to $\sim 0.02$ ps. These statistical errors can be expected to be comparable in size to the likely systematic errors for this measurement. 


\section{Chapter 8}

\section{Conclusions}

\subsection{B physics and CP violation measurements at $\mathrm{D} \emptyset$}

The complete and fully commissioned $\mathrm{D} \varnothing$ detector will be used for a large number of $B$ physics and $\mathrm{CP}$ violation measurements. The highest profile of these is the measurement of the CKM angle $\beta$ from the decay $B^{0} \rightarrow J / \psi K_{S}$, but some of the other planned measurements are described in the following sections. Many $B$ physics channels involve fully reconstructing $B$ mesons, so the measurement of the $B^{+}$and $B_{d}^{0}$ lifetimes as described in chapter 7 will be an early target in order to develop the skills and techniques needed to carry out the ore complicated analyses.

\subsection{Measuring $\sin 2 \beta$}

As described in section 2.5.3, CP violation appears in $B^{0} / \bar{B}^{0}$ decays to the (approximate) CP eigenstate $J / \psi K_{S}$. Decays via $B^{0} \rightarrow J / \psi K_{S}$ interfere with those via 
$B^{0} \rightarrow \bar{B}^{0} \rightarrow J / \psi K_{S}$ to give a time dependent decay asymmetry

$$
\mathcal{A}_{\mathrm{CP}}(t)=\frac{\bar{B}^{0}(t)-B^{0}(t)}{\bar{B}^{0}(t)+B^{0}(t)}=\sin 2 \beta \sin \Delta m_{d} t
$$

where $B^{0}(t)\left(\bar{B}^{0}(t)\right)$ is the number of decays to $J / \psi K_{S}$ at proper time $t$ given that the meson was originally produced as a $B^{0}\left(\bar{B}^{0}\right)$ at time $t=0$. The difference in the CP phase between the two paths is responsible for the $\sin 2 \beta$ factor, and the $B \bar{B}$ flavour oscillation appears as the time dependence on the mass difference $\Delta m_{d}$ between the two $B^{0}$ mass states.

There are two major components to measuring $\mathcal{A}_{\mathrm{CP}}(t)$ - fully reconstructing the $J / \psi K_{S}$ final state, and tagging the initial flavour of the $B^{0}$.

\subsubsection{Flavour tagging}

Flavour tagging involves determining the flavour of a $B$ meson, either at its production, or at its decay. There are a number of different methods to do this. Since the dominant $b$ quark production mechanism creates $b \bar{b}$ pairs the flavours of a pair of $B$ mesons can be assumed to be opposite at the time of their production. This means that it is possible to determine the flavour of a $B$ meson of interest either directly ('same side tagging') or by determining the flavour of the other $B$ meson ('opposite side tagging').

At DØ, there are two methods of opposite side tagging. One method, 'lepton tagging', uses a lepton coming from a semileptonic decay of the other $B$ hadron. The charge of this lepton depends on the flavour of the $b$ quark - an $\ell^{-}$comes from a $b \rightarrow c \ell^{-} \bar{\nu} X$ decay, while an $\ell^{+}$comes from a $\bar{b}$ quark. The second method, "jet charge tagging', makes use of the hadron jet coming from the $b$ or $\bar{b}$ quark. The momentum weighted sum of the charges of the particles in the jet should be the same sign as the charge of the $b$ quark responsible for the jet ${ }^{1}$. There is a single

\footnotetext{
${ }^{1}$ There is another method of opposite side tagging - using the charge of a kaon from the decay
} 
method of same side tagging, which is to exploit the correlation between the flavour of the $B$ meson of interest and the charge of particles produced in association with it. These correlations are due to $b$ quark hadronisation and to $B^{* *} \rightarrow B$ decays.

The power of a tagging method is described by its 'effective tagging efficiency' $\varepsilon \mathcal{D}^{2}$, where $\varepsilon$ is the efficiency of the tag, and $\mathcal{D}$ is called the 'dilution'. The dilution is defined as

$$
\mathcal{D}=\frac{N_{R}-N_{W}}{N_{R}+N_{W}}
$$

where $N_{R}$ is the number of correctly tagged events and $N_{W}$ the number of incorrectly tagged events ${ }^{2}$. The dilution is related to the probability $P_{R}$ that a tag is correct and the probability $P_{W}=1-P_{R}$ that it is incorrect

$$
\mathcal{D}=2 P_{R}-1=1-2 P_{W}
$$

The value of $\varepsilon \mathcal{D}^{2}$ gives a guide to the statistical power of a tagging method. For example, for a measurement of the asymmetry between $B^{0}$ and $\bar{B}^{0}$ decays, the measured asymmetry is

$$
\mathcal{A}_{\text {meas }}=\frac{N_{B^{0}}-N_{\bar{B}^{0}}}{N_{B^{0}}+N_{\bar{B}^{0}}}
$$

where $N_{B^{0}}$ is the number of events tagged as $B^{0}$, and $N_{\bar{B}^{0}}$ is the number tagged as $\bar{B}^{0}$. The true asymmetry is

$$
\mathcal{A}_{\text {true }}=\frac{N_{B^{0}}^{T}-N_{\bar{B}^{0}}^{T}}{N_{B^{0}}^{T}+N_{\bar{B}^{0}}^{T}}
$$

chain $b \rightarrow c \rightarrow s$. A $K^{-}$signals a $b$, and a $K^{+}$a $\bar{b}$. Without some form of particle identification to distinguish kaons from pions this method is not possible at $\mathrm{D} \varnothing$.

${ }^{2}$ Calling $\mathcal{D}$ the 'dilution' is slightly misleading. A perfect tagging method has $\mathcal{D}=1$, while one right $50 \%$ of the time (useless, in other words) has $\mathcal{D}=0$. A large dilution is a desirable property for a tagging algorithm. 
where $N_{B^{0}}^{T}$ and $N_{\bar{B}^{0}}^{T}$ are the true numbers of each flavour. The efficiency is

$$
\varepsilon=\frac{N_{B^{0}}+N_{\bar{B}^{0}}}{N_{B^{0}}^{T}+N_{\bar{B}^{0}}^{T}}
$$

and the measured number of events is related to the true number by

$$
N_{B^{0}}=\varepsilon\left(P_{R} N_{B^{0}}^{T}+P_{W} N_{\bar{B}^{0}}^{T}\right) \quad N_{\bar{B}^{0}}=\varepsilon\left(P_{R} N_{\bar{B}^{0}}^{T}+P_{W} N_{B^{0}}^{T}\right)
$$

The measured and true asymmetries are therefore related by

$$
\mathcal{A}_{\text {meas }}=\frac{\varepsilon\left(2 P_{R}-1\right)\left(N_{B^{0}}^{T}-N_{\bar{B}^{0}}^{T}\right)}{\varepsilon\left(N_{B^{0}}^{T}+N_{\bar{B}^{0}}^{T}\right)}=\mathcal{D} \mathcal{A}_{\text {true }}
$$

so the effect of $\mathcal{D}$ is to dilute the true asymmetry to the measured value. The statistical error on the true asymmetry is given by

$$
\sigma^{2}\left(\mathcal{A}_{\text {true }}\right)=\frac{1}{\mathcal{D}^{2}} \sigma^{2}\left(\mathcal{A}_{\text {meas }}\right) \sim \frac{1}{\varepsilon \mathcal{D}^{2} N}
$$

where $N$ is the total number of events in the sample, and so $\varepsilon N$ is the number of tagged events. $\varepsilon \mathcal{D}^{2} N$ is therefore the effective statistics of the data sample - the equivalent number of perfectly tagged events.

The tagging dilution can be measured from the data itself. There are two ways to do this. The first is combined with measuring $B_{d}^{0} \bar{B}_{d}^{0}$ mixing — the $B^{0} \bar{B}^{0}$ asymmetry is reduced by a factor of $\mathcal{D}$ (as in equation (8.8)), so a fit to the sinusoidal asymmetry distribution will give $\mathcal{D}$ as its magnitude. Alternatively, the dilution can be measured directly. For opposite side tags this can be done with $B^{+}$decays, such as $B^{+} \rightarrow$ $J / \psi K^{+}$. The sign of the kaon gives the flavour of the $B$ and can be compared to the result of the opposite side tag.

Table 8.1 gives the effective tagging efficiencies achieved by CDF in Run I, and the estimated efficiencies for both CDF and DØ in Run II, extrapolated from the CDF Run I results. The large pseudorapidity range covered by the $\mathrm{D} \varnothing$ muon system and calorimeter are a particular advantage for $\mu$ and $e$ identification, and so for lepton 
tagging, while the forward tracking allows jet charge tagging over a large range.

\begin{tabular}{|c|c|c|c|c|}
\hline Tag & $\begin{array}{l}\text { CDF Run I } \\
\varepsilon \mathcal{D}^{2}(\%)\end{array}$ & $\begin{array}{l}\text { CDF Run II } \\
\text { expected } \\
\varepsilon \mathcal{D}^{2}(\%)\end{array}$ & DØ difference & $\begin{array}{l}\text { DØ Run II } \\
\text { expected } \\
\varepsilon \mathcal{D}^{2}(\%)\end{array}$ \\
\hline Same side & $1.8 \pm 0.4 \pm 0.3$ & 2.0 & same & 2.0 \\
\hline Lepton & $0.9 \pm 0.1 \pm 0.1$ & 1.7 & $\mu, e$ ID coverage & 3.1 \\
\hline Jet charge & $0.8 \pm 0.1 \pm 0.1$ & 3.0 & Forward tracking & 4.7 \\
\hline Kaon & - & 2.4 & no $K$ ID & - \\
\hline Combined & 3.5 & 9.1 & & 9.8 \\
\hline
\end{tabular}

Table 8.1: Measured effective tagging efficiency for CDF Run I, and the predicted (extrapolated from the measured values) efficiencies for CDF and DØ in Run II [8, 89]

\section{$8.2 .2 \sin 2 \beta$}

Measuring $\sin 2 \beta$ involves reconstructing $B^{0}$ mesons through the decay channel $B^{0} \rightarrow J / \psi K_{S} . J / \psi$ candidates are formed in the manner described in section 7.3 and $K_{S}$ are formed from combining $\pi^{+}$and $\pi^{-}$tracks to a common vertex (see figure 8.1). The $J / \psi$ and $K_{S}$ candidates are then combined together to form $B^{0}$ candidates. In addition a flavour tag must be made for each event using one of the algorithms in the previous section. Using this tagged sample, it is then possible to form a bin-by-bin asymmetry (compare with equation (8.1))

$$
\mathcal{A}(c t)=\frac{N^{-}(c t)-N^{+}(c t)}{N^{-}(c t)+N^{+}(c t)}
$$

where $c t$ is the proper decay length, and $N^{\mp}(c t)$ is the number of $\bar{B}^{0}\left(B^{0}\right)$ in each proper time bin. The value of $\sin 2 \beta$ can then be extracted by fitting the function $\mathcal{D} \sin 2 \beta \sin \Delta m_{d} t$ where $\mathcal{D}$ is the tagging dilution (which is a combination of the dilutions of the different tagging methods, and must be separately determined). $\Delta m_{d}$ is the mixing parameter - although this can be allowed to float in the fit it 


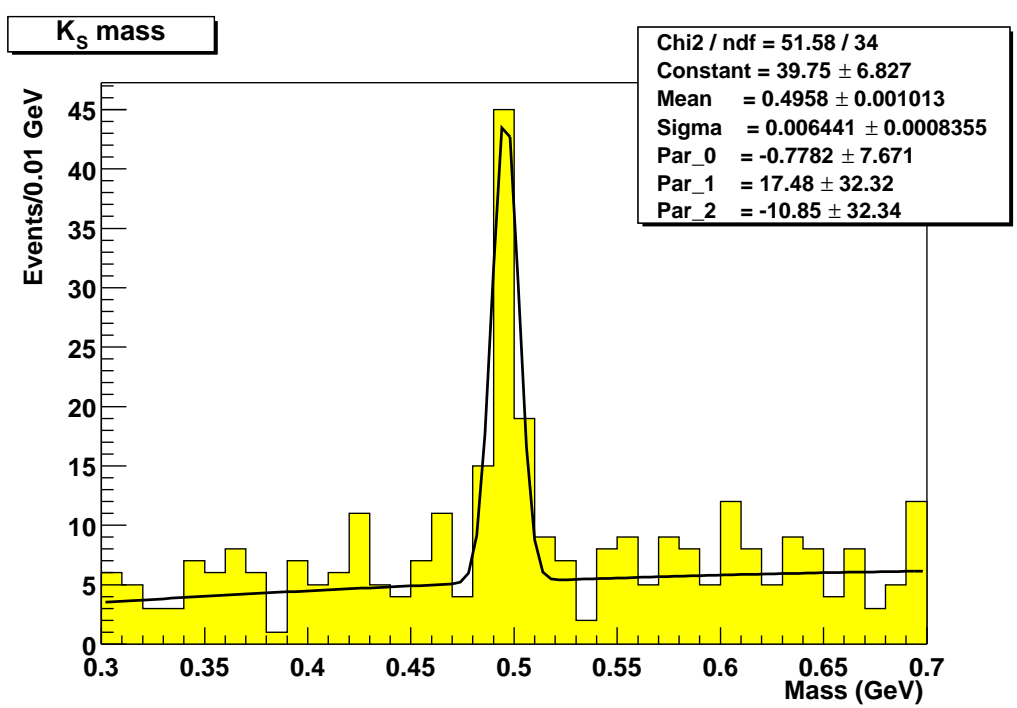

Figure 8.1: Reconstructed $K_{S}$ invariant mass from 1000 simulated $B^{0}$ decays. The $K_{S}$ were formed by vertex constraining pairs of tracks (assumed to be $\pi^{+} \pi^{-}$) which had an invariant mass close to the $K_{S}$ mass. To reduce the combinatoric background the impact parameter significance of each of the tracks was required to be greater than 3 .

is already well measured and alternatively can be fixed to its best value.

The predicted error on the measurement is given by the equation [90]

$$
\sigma(\sin 2 \beta) \sim e^{x_{d}^{2} \Gamma^{2} \sigma_{t}^{2}} \sqrt{\frac{1+4 x_{d}^{2}}{2 x_{d}^{2}}} \frac{1}{\varepsilon \mathcal{D}^{2} N} \sqrt{1+\frac{B}{S}}
$$

where $\Gamma$ and $x_{d}=\Delta m_{d} / \Gamma$ are the $B_{d}^{0}$ decay width and mixing parameter, $\sigma_{t}$ is the proper time measurement resolution, $S / B$ is the signal to background ratio, and $N$ is the total number of events.

Previous studies [90] suggest that $\mathrm{D} \varnothing$ will be able to measure $\sin 2 \beta$ with an accuracy of about 0.04 in two years running using the dimuon mode, and to a similar level in the dielectron mode. 


\subsection{Other $B$ and CP measurements at DØ}

Apart from the measurement of $\sin 2 \beta$, there are a number of other $B$ physics and $\mathrm{CP}$ violation measurements that will be made at $\mathrm{D} \varnothing$.

\subsection{1 $\quad B_{s}$ mixing}

$B_{s}^{0} \bar{B}_{s}^{0}$ mesons oscillate in the same way as $B_{d}^{0} \bar{B}_{d}^{0}$ mesons (or as $K^{0} \bar{K}^{0}$ mesons) do. The current upper limit on the mass difference between the oscillating states (expressed as the mixing frequency) is $\Delta m_{s}>13.1 \mathrm{ps}^{-1}$ [12]. The value of $\Delta m_{s}$ is interesting because the ratio $\Delta m_{d} / \Delta m_{s}$ gives a theorectically clean measurement of $\left|V_{t d}\right|^{2} /\left|V_{t s}\right|^{2}$ [91], and so helps constrain the CKM parameters $\rho$ and $\eta$. This measurement, using $B_{s}$ mesons, is not possible at the $B$ factory detectors because the $\Upsilon(4 S)$ resonance is below the threshold to produce $B_{s}$ pairs.

Measuring $\Delta m_{s}$ is difficult because the high oscillation frequency requires very good momentum and decay length resolution to resolve the oscillations as a function of proper time. It is also necessary to tag both the initial and final $B_{s}$ flavour. Below $\Delta m_{s} \sim 18 \mathrm{ps}^{-1}$ semileptonic decays of the $B_{s}$ can be used. The final state lepton provides a trigger and also gives the decay flavour tag. If $\Delta m_{s}$ is larger then it becomes necessary to use decays such as $B_{s} \rightarrow D_{s} \pi$ and $B_{s} \rightarrow D_{s} \pi \pi \pi$, with $D_{s} \rightarrow \phi \pi$ or $K^{*} K$. These fully hadronic decays do not provide a useable trigger as there is not enough Level 1 trigger bandwidth to trigger only on two low $p_{T}$ tracks to catch these events, and so the trigger signal must come from the semileptonic decay of the other $B$ in the event.

The expected reach of $\mathrm{D} \varnothing$ in two years running is to be able to measure $\Delta m_{s}$ values up to $\sim 22 \mathrm{ps}^{-1}[90]$. 


\subsection{2 $B_{d}^{0} \rightarrow \pi^{+} \pi^{-}, B_{s}^{0} \rightarrow K^{+} K^{-}$}

The decay $B_{d}^{0} \rightarrow \pi^{+} \pi^{-}$was once seen as a key mode to measure the CKM angle $\alpha$, but was later found to suffer significant contamination from penguin Feynman diagrams which make extracting the value of $\alpha$ much more difficult. However, it is possible to use a combination of the $\mathrm{CP}$ asymmetries from $B_{d}^{0} \rightarrow \pi^{+} \pi^{-}$and $B_{s}^{0} \rightarrow K^{+} K^{-}$to extract both angles $\alpha$ and $\gamma[32]$.

Measuring these asymmetries at $\mathrm{D} \varnothing$ is difficult. The first problem is that triggering on these hadronic final states is difficult - again it is necessary to trigger off a semileptonic decay from the other $B$. The lepton does at least provide a good initial state flavour tag for the event. Once an event has been triggered on, the second problem is with the reconstruction. Lacking any $K / \pi$ particle identification, D $\varnothing$ cannot separate $B_{d}^{0} \rightarrow \pi^{+} \pi^{-}, B_{s}^{0} \rightarrow K^{+} K^{-}, B_{d}^{0} \rightarrow K \pi$ and $B_{s}^{0} \rightarrow K \pi$ and they will all reconstruct to the same mass range. However, the $K \pi$ modes show no CP asymmetry, while the $B_{d}^{0} \rightarrow \pi^{+} \pi^{-}$and $B_{s}^{0} \rightarrow K^{+} K^{-}$have different CP asymmetries, so it may be possible to separate the $\mathrm{CP}$ violating effect out and determine the asymmetries to an estimated $20 \%$ level [90].

\subsection{3 $\quad B_{s} \rightarrow J / \psi \phi$}

The decay $B_{s} \rightarrow J / \psi \phi$ has only a very small $\mathrm{CP}$ violating effect in the Standard Model, so it is a good place to look for non-Standard Model effects. Additionally, it is possible to measure the lifetime difference between the $\mathrm{CP}=+1$ and $\mathrm{CP}=-1$ eigenstates of the $B_{s}$. This difference is predicted to be up to $20 \%$ of the mean $B_{s}$ lifetime. The final state of the decay will be a mixture of the two CP eigenstates, and the two states can be separated by the different angular distributions of the decay products $J / \psi \rightarrow \ell^{+} \ell^{-}$and $\phi \rightarrow \pi^{+} \pi^{-}$. A fit to the decay lengths and the angular distributions can then separate the lifetimes of the two CP components and so determine their difference. 


\subsubsection{Other $B$ measurements}

The planned $b$ physics programme at D $\varnothing$ includes many other measurements. These include the $b \bar{b}$ cross-section, rare (non $b \rightarrow c$ ) decays, and the spectroscopy and lifetime measurements of the $B_{c}$ and $\Lambda_{b}[90]$.

\subsection{Summary}

The D $\varnothing$ detector is about to begin full physics data taking for Run II at the Tevatron. The upgraded detector requires completely new reconstruction and trigger software to make use of its new capabilities. An important first stage of the software is to take the raw data from the sub detectors and turn it into a useable form for the later stages. It is particularly important that the trigger version of this does its work as quickly and efficiently as possible, as it is limited in the time available to process events. The silicon and fibre tracker software components described in this thesis are fully able to achieve this.

DØ has a full programme for $B$ physics. The first steps once data is available is to develop and understand the techniques required to carry out the analyses. The measurement of the $B^{+}$and $B^{0}$ lifetimes provides a opportunity to develop the methods needed to fully reconstruct $B$ mesons and their proper lifetimes, such as mass constrained fitting. This is an important component of the measurement of the CKM angle $\beta$ from the decay $B^{0} \rightarrow J / \psi K_{S}$, as well as many other measurements.

DØ provides an important $B$ physics capability, which is both competitive with, and complementary to, other existing $B$ physics facilities. The forthcoming results will either significantly constrain the Standard Model picture of CP violation, or demonstrate that it is incorrect and so show the existence of new physics. 


\section{Appendix A}

\section{Likelihood fitting}

\section{A.1 The method of maximum likelihood}

The method of maximum likelihood $[92,93]$ is a technique for estimating the parameters of a probability density function given a sample of data that is believed to come from that distribution. If the probability density function $f(\mathbf{x} \mid \boldsymbol{\theta})$ has a set of parameters $\boldsymbol{\theta}$, and the measurement of the random variables $\mathbf{x}$ has been repeated $n$ times (giving a set of data $\mathbf{x}_{1}, \mathbf{x}_{2}, \ldots \mathbf{x}_{n}$ ), then the likelihood function $\mathcal{L}$ is given by the product of the likelihood of each individual set of parameters $\mathbf{x}_{i}$

$$
\mathcal{L}=\prod_{i=1}^{n} \mathcal{L}_{i}=\prod_{i=1}^{n} f\left(\mathbf{x}_{i} \mid \boldsymbol{\theta}\right)
$$

The likelihood function is the joint probability density for the $n$ measurements of the variables $\mathbf{x}$ for a given $\boldsymbol{\theta}$.

The maximum likelihood estimators for the parameters $\boldsymbol{\theta}$ are those which maximise the likelihood function. (These are the parameters which make the given data points $\mathbf{x}$ most likely.) It is usually more computationally convenient to consider 
the logarithm of the likelihood - this is called the log-likelihood function and has maxima at the same points as the likelihood function

$$
\log \mathcal{L}=\sum_{i=1}^{n} \log f(\mathbf{x} \mid \boldsymbol{\theta})
$$

The probability density function $f(\mathbf{x})$ must be normalised correctly - in particular when the data covers a restricted range then the total probability must integrate to 1 over that range rather than the full range of the function. This becomes important when considering a probability density function consisting of the sum of other probability density functions.

With a simple likelihood function it may be possible to find its maximum by algebra, but with more complicated functions it is usually necessary to use a numerical software package such as MINUIT [94] to find the maximum.

\section{A.2 Extended maximum likelihood}

In the normal log likelihood, the probability density function is normalised to 1 . The extended maximum likelihood method uses a different distribution function where its integral is the number of events in the data, which is considered to be an additional random variable. The number of observations (such as the number of events found) is often a Poisson distributed variable with mean $\nu$. The extended likelihood function is

$$
\mathcal{L}(\mathbf{x} \mid \nu, \boldsymbol{\theta})=\frac{e^{-\nu}}{n !} \nu^{n} \prod_{i=1}^{n} f(\mathbf{x} \mid \boldsymbol{\theta})
$$

This is useful when the overall probability density function is the superposition of 
several components

$$
f(\mathbf{x} \mid \boldsymbol{\theta})=\sum_{j=1}^{m} \eta_{j} f_{j}(\mathbf{x})
$$

where there are $m$ components and $\eta_{i}$ is the fractional contribution of each, and so

$$
\sum_{j=1}^{m} \eta_{j}=1
$$

This can be handled by using the extended maximum likelihood. Taking the logarithm of equation (A.3) gives

$$
\log \mathcal{L}(\mathbf{x} \mid \nu, \boldsymbol{\theta})=-\nu+\sum_{i=1}^{n} \log \left(\sum_{j=1}^{m} \nu \eta_{j} f_{j}(\mathbf{x} \mid \boldsymbol{\theta})\right)
$$

By defining $\mu_{i}=\eta_{i} \nu$ as the expected number of events of type $i$ the log likelihood becomes

$$
\log \mathcal{L}(\mathbf{x} \mid \boldsymbol{\mu}, \boldsymbol{\theta})=-\sum_{j=1}^{m} \mu_{j}+\sum_{i=1}^{n} \log \left(\sum_{j=1}^{m} \mu_{j} f_{j}(\mathbf{x} \mid \boldsymbol{\theta})\right)
$$

\section{A.3 Probability density functions}

\section{Exponential decay}

The probability density function for an exponential decay with mean lifetime $\lambda$ is

$$
\mathcal{E}(x \mid \lambda)=\frac{1}{\lambda} e^{-\frac{x}{\lambda}}
$$




\section{Gaussian distribution}

The probability density function for a Gaussian distribution with mean $\mu$ and standard deviation $\sigma$ is

$$
\mathcal{G}(x \mid \mu, \sigma)=\frac{1}{\sqrt{2 \pi \sigma^{2}}} e^{-\frac{(x-\mu)^{2}}{2 \sigma^{2}}}
$$

\section{Exponential decay with Gaussian resolution function}

This is the probability density function of an exponential decay function smeared with a Gaussian resolution function at each point.

$$
\begin{aligned}
\mathcal{F}(x \mid \lambda, \sigma) & =\frac{1}{\lambda \sqrt{2 \pi \sigma^{2}}} \int_{0}^{\infty} e^{-u / \lambda} e^{-(u-x)^{2} / 2 \sigma^{2}} d u \\
& =\frac{1}{\lambda \sqrt{2 \pi \sigma^{2}}} e^{-x / \lambda+\sigma^{2} / 2 \lambda^{2}} \int_{0}^{\infty} e^{-\left(u-\left(x-\sigma^{2} / \lambda\right)\right)^{2} / 2 \sigma^{2}} d u \\
& =\frac{1}{2 \lambda} e^{-x / \lambda+\sigma^{2} / 2 \lambda^{2}}\left[1+\operatorname{erf}\left(\left(\frac{x}{\sigma}-\frac{\sigma}{\lambda}\right) / \sqrt{2}\right)\right]
\end{aligned}
$$

where erf $x$ is the error function

$$
\operatorname{erf}(x)=\frac{2}{\sqrt{\pi}} \int_{0}^{x} e^{-y^{2}} d y
$$

Normalising this function over a restricted range requires the indefinite integral, which can be obtained by integrating by parts

$$
\begin{aligned}
\int \mathcal{F}(x \mid \lambda, \sigma) d x= & -\frac{1}{2} e^{-x / \lambda+\sigma^{2} / 2 \lambda^{2}}\left[1+\operatorname{erf}\left(\left(\frac{x}{\sigma}-\frac{\sigma}{\lambda}\right) / \sqrt{2}\right)\right] \\
& +\frac{1}{2} \int e^{-x / \lambda+\frac{1}{2} \sigma^{2} / \lambda^{2}} \frac{2}{\sqrt{\pi}} \frac{1}{\sqrt{2 \sigma^{2}}} e^{-\frac{1}{2}(x / \sigma-\sigma / \lambda)^{2}} d x \\
= & -\lambda \mathcal{F}(\lambda \mid x, \sigma)+\frac{1}{2} \frac{2}{\sqrt{\pi}} \frac{1}{\sqrt{2 \sigma^{2}}} \int e^{-x^{2} / 2 \sigma^{2}} d x \\
= & \frac{1}{2}\left(1+\operatorname{erf}\left(\frac{x}{\sqrt{2 \sigma^{2}}}\right)\right)-\lambda \mathcal{F}(x \mid \lambda, \sigma)
\end{aligned}
$$




\section{References}

[1] Fermilab Beams Division, Run II Handbook, 2001.

[2] DØ Collaboration, S. Abachi et al., "The DØ detector", Nucl. Instrum. Meth. A338 (1994) 185-253.

[3] DØ Collaboration, "The DØ upgrade: The detector and its physics". Fermilab Pub-96/357-E, July, 1996.

[4] DØ Collaboration, S. Abachi et al., "The DØ upgrade", Nucl. Instrum. Meth. A408 (1998) 103-109.

[5] J. Ellison, "The D $\varnothing$ detector upgrade and physics program", arXiv:hep-ex/0101048.

[6] C. S. Wu, E. Ambler, R. W. Hayward, D. D. Hoppes, and R. P. Hudson, "Experimental test of parity conservation in beta decay", Phys. Rev. 105 (1957) 1413-1414.

[7] J. H. Christenson, J. W. Cronin, V. L. Fitch, and R. Turlay, "Evidence for the $2 \pi$ decay of the $K_{2}^{0}$ meson", Phys. Rev. Lett. 13 (1964) 138-140.

[8] CDF Collaboration, T. Affolder et al., "A measurement of $\sin (2 \beta)$ from $B \rightarrow J / \psi K_{\mathrm{S}}^{0}$ with the CDF detector", Phys. Rev. D61 (2000) 072005, arXiv:hep-ex/9909003. 
[9] BABAR Collaboration, B. Aubert et al., "Measurement of CP violating asymmetries in $B^{0}$ decays to CP eigenstates", Phys. Rev. Lett. 86 (2001) 2515-2522, arXiv:hep-ex/0102030.

[10] Belle Collaboration, A. Abashian et al., "Measurement of the CP violation parameter $\sin 2 \phi_{1}$ in $B_{d}^{0}$ meson decays", Phys. Rev. Lett. 86 (2001) 2509-2514, arXiv:hep-ex/0102018.

[11] M. N. Achasov et al., "Upper limit on the $K_{S} \rightarrow 3 \pi^{0}$ decay", Phys. Lett. B459 (1999) 674-678, arXiv:hep-ex/9907004.

[12] Particle Data Group Collaboration, D. E. Groom et al., "Review of particle physics", Eur. Phys. J. C15 (2000) 1. 2001 partial update http://pdg. Ibl.gov/.

[13] G. C. Branco, L. Lavoura, and J. P. Silva, CP Violation. Clarendon Press, Oxford, 1999.

[14] Y. Nir, "CP violation in and beyond the Standard Model", arXiv:hep-ph/9911321. Lectures given at 27th SLAC Summer Institute on Particle Physics: CP Violation in and Beyond the Standard Model (SSI 99), Stanford, California, 7-16 Jul 1999.

[15] Y. Nir, "CP violation: A new era", arXiv:hep-ph/0109090. Lectures given at 55th Scottish Universities Summer School in Physics on Heavy Flavor Physics, St. Andrews, Scotland, 7-23 Aug 2001.

[16] The LEP Collaborations: ALEPH, DELPHI, L3, and OPAL, "Electroweak parameters of the $Z^{0}$ resonance and the Standard Model", Phys. Lett. B276 (1992) 247-253.

[17] DØ Collaboration, S. Abachi et al., "Search for a fourth generation charge -1/3 quark via flavor changing neutral current decay", Phys. Rev. Lett. 78 (1997) 3818-3823, arXiv:hep-ex/9611021. 
[18] CDF Collaboration, T. Affolder et al., "Search for a fourth-generation quark more massive than the $Z^{0}$ boson in $p$ anti- $p$ collisions at $\sqrt{s}=1.8 \mathrm{TeV}$ ", Phys. Rev. Lett. 84 (2000) 835-840, arXiv:hep-ex/9909027.

[19] M. Kobayashi and T. Maskawa, "CP violation in the renormalizable theory of weak interaction", Prog. Theor. Phys. 49 (1973) 652-657.

[20] L. Wolfenstein, "Parametrization of the Kobayashi-Maskawa matrix", Phys. Rev. Lett. 51 (1983) 1945.

[21] R. Aleksan, B. Kayser, and D. London, "Determining the quark mixing matrix from CP violating asymmetries", Phys. Rev. Lett. 73 (1994) 18-20, arXiv: hep-ph/9403341.

[22] Y. Grossman, Y. Nir, and R. Rattazzi, "CP violation beyond the Standard Model", Adv. Ser. Direct. High Energy Phys. 15 (1998) 755-794, arXiv:hep-ph/9701231.

[23] M. L. Mangano, "Heavy-quark production in hadronic collisions", in Proceedings of the International School of Physics, "Enrico Fermi": Heavy Flavor Physics - A Probe Of Nature's Grand Design, Varenna, 199\%, pp. 95-137. 1998. arXiv:hep-ph/9711337.

[24] M. Paulini, "B lifetimes, mixing and CP violation at CDF", Int. J. Mod. Phys. A14 (1999) 2791, arXiv:hep-ex/9903002.

[25] D $\varnothing$ Collaboration, B. Abbott et al., "The $b \bar{b}$ production cross section and angular correlations in $p \bar{p}$ collisions at $\sqrt{s}=1.8 \mathrm{TeV} "$, Phys. Lett. B487 (2000) 264-272, arXiv:hep-ex/9905024.

[26] M. L. Mangano, P. Nason, and G. Ridolfi, "Heavy quark correlations in hadron collisions at next-to-leading order", Nucl. Phys. B373 (1992) 295-345.

[27] I. I. Bigi, "Lifetimes of heavy-flavour hadrons: Whence and whither?", Nuovo Cim. 109A (1996) 713-726, arXiv:hep-ph/9507364. 
[28] M. Neubert and C. T. Sachrajda, "Spectator effects in inclusive decays of beauty hadrons", Nucl. Phys. B483 (1997) 339-370, arXiv:hep-ph/9603202.

[29] Y. Nir, "CP violation in $B$ decays", arXiv:hep-ph/9904271. Lectures given at NATO Advanced Study Institute and Cargese Summer School on Flavor and Gauge Hierarchies, Cargese, France, 20 Jul - 1 Aug 1998 and 1st Particle Physics Winter School: B and CP Within and Beyond the Standard Model, Seoul, Korea, 22-26 Feb 1999.

[30] BABAR Collaboration, B. Aubert et al., "Search for direct CP violation in quasi-two-body charmless $B$ decays", arXiv:hep-ex/0109006.

[31] Belle Collaboration, K. Abe et al., "Search for direct CP violation in $B \rightarrow K \pi$ decays", Phys. Rev. D64 (2001) 071101, arXiv:hep-ex/0106095.

[32] R. Fleischer, "New strategies to extract $\beta$ and $\gamma$ from $B_{d} \rightarrow \pi^{+} \pi^{-}$and $B_{s} \rightarrow K^{+} K^{-"}$, Phys. Lett. B459 (1999) 306-320, arXiv:hep-ph/9903456.

[33] TASSO Collaboration, W. Braunschweig et al., "A study of jets from $b$ quarks produced in $e^{+} e^{-}$annihilations at $\sqrt{s}=35 \mathrm{GeV}$ to $46 \mathrm{GeV}$ ", Z. Phys. C42 (1989) 17.

[34] OPAL Collaboration, K. Ackerstaff et al., "Investigation of CP violation in $B^{0} \rightarrow J / \psi K_{\mathrm{S}}^{0}$ decays at LEP", Eur. Phys. J. C5 (1998) 379-388, arXiv:hep-ex/9801022.

[35] Belle Collaboration, K. Abe et al., "Observation of large CP violation in the neutral B meson system", Phys. Rev. Lett. 87 (2001) 091802, arXiv:hep-ex/0107061.

[36] BaBAR Collaboration, B. Aubert et al., "Observation of CP violation in the $B^{0}$ meson system", Phys. Rev. Lett. 87 (2001) 091801, arXiv:hep-ex/0107013. 
[37] A. Hocker, H. Lacker, S. Laplace, and F. Le Diberder, "A new approach to a global fit of the CKM matrix", Eur. Phys. J. C21 (2001) 225-259, arXiv:hep-ph/0104062.

[38] DØ Collaboration, "Run IIb trigger conceptual design report", October, 2001.

[39] M. T. P. Roco, "The silicon microstrip tracker for the DØ upgrade", Nucl. Phys. Proc. Suppl. 78 (1999) 275-280.

[40] "DØ silicon tracker technical design report". DØ Note 2169, July, 1994.

[41] M. R. Wayne, "Visible light photon counters and the DØ scintillating fiber tracker", Nucl. Instrum. Meth. A387 (1997) 278-281.

[42] G. C. Blazey, "The DØ Run II trigger". FERMILAB-CONF-97-395-E, Talk given at 10th IEEE Real-Time Computer Applications in Nuclear, Particle and Plasma Physics (RT 97), Beaune, France, 22-26 Sep 1997.

[43] A. Boehnlein, G. Brooijmans, D. Claes, and M. Souza, "Description of the DØ L3 Trigger software components". DØ Note 3630, April, 1999.

[44] I. Blackler and G. Davies. Private communication, November, 2001.

[45] J. Kowalkowski, D. Chakraborty, H. Greenlee, Q. Li, and G. Watts, "Framework users guide", February, 1999.

[46] DØ Collaboration, "DØ Run 2B silicon detector upgrade technical design report", October, 2001.

[47] "Beginner's guide to the SVXIIe". FERMILAB-TM-1892, October, 1996.

[48] A. Juste, "The readout system for the silicon microstrip tracker of the upgraded DØ detector", Nucl. Instrum. Meth. A461 (2001) 155-157.

[49] D. Cohen, "On holy wars and a plea for peace", Internet Engineering Note 137, USC/Information Sciences Institute, April, 1980. (The phrases big-endian and little-endian originally came from [50]). 
[50] J. Swift, Travels into Several Remote Nations of the World by Lemuel Gulliver. Benjamin Motte, London, 1726.

[51] "VME Readout Buffer". Fermilab-ESE-SVX-950719, February, 2001.

[52] M. Utes, "SVX sequencer board". DØ Engineering Note Number 3823.110-EN-480, August, 2000.

[53] J. E. McDonald, "Gray code and binary conversion". DØ Note 3652, May, 1999.

[54] Source code and documentation available from http://www-d0.fnal.gov/d0dist/dist/packages/l3base/devel/.

[55] Source code available from http://www-d0.fnal.gov/d0dist/dist/packages/unpack_reco/devel/.

[56] F. Blekman, "Numbering schemes for the DØ SMT". DØ Note 3899, August, 2001 .

[57] Source code available from http://www-d0.fnal.gov/d0dist/dist/packages/smtraw/devel/.

[58] Source code available from http://www-d0.fnal.gov/d0dist/dist/packages/l3f smtunptool/devel/.

[59] D. Bauer. Private communication, July, 2001.

[60] A. Juste. Private communication, October, 2001.

[61] D. Bauer and R. Illingworth. DØ Note in preparation.

[62] D. Bauer and R. Illingworth, "L3 trigger: Clustering in the SMT". DØ Note 3822, January, 2001.

[63] M. Narain, "DØ SMT software numbering scheme". http://www-d0.fnal. gov/〜meena/d0_private/silicon/coords/smt-numb/sld001.htm. 
[64] E. Barberis, E. Shabalina, S. Kulik, A. Solomin, and T. Trippe, "Summary of modifications to the SMT software readout/geometry". DØ Note 3780, October, 2000.

[65] Y. Gershtein et al., "SMT barrel assembly". DØ Note 3849, March, 2001.

[66] E. Barberis. Private communication, April, 2000.

[67] M. T. P. Roco, "Analysis of the SMT beam tests data". DØ Note 3405, February, 1998.

[68] D. Bauer. Private communication, March, 2001.

[69] E. Barberis and Y. Kulik. Private communication, April, 2001.

[70] CTT Group, "CTT TDR: Technical design report for the upgrade L1/L2 Tracking Trigger, including Central Fiber Tracker, Central Preshower Detector, Forward Preshower Detector, Forward Proton Detector". DØ Note 3551, January, 1999.

[71] DØ Collaboration, D. Adams et al., "VLPC characterization for the DØ upgrade", in Proceedings of the Eighth Meeting of the Division of Particles and Fields of the American Physical Society (DPF'94), pp. 1873-1876. 1994. arXiv:hep-ex/9410011.

[72] J. T. Anderson, "D-Zero Central Fiber Tracker 8-MCM Analog Front End board". CFT Note A1000107, January, 2000.

[73] B. Hoeneisen and P. Rubinov, "Replace the SIFT, MCM, or AFE? Design of a 'new SIFT' circuit”. DØ Note 3773, August, 2000.

[74] B. Hoeneisen and P. Rubinov, "Design of the new MCM". DØ Note 3898, August, 2001.

[75] J. T. Anderson, "SVX and Virtual SVX operation in the Analog Front End board". CFT Note A1010913B, September, 2001. 
[76] Imperial College DØ Group, "L3TCFTTrack - a Level-3 CFT Tracking Tool". DØ Note 3779, September, 2000.

[77] R. Beuselinck. Private communication, September, 2001.

[78] R. Frühwirth, M. Regler, R. K. Bock, H. Grote, and D. Notz, Data Analysis Techniques for High-Energy Physics, ch. 3.4. Cambridge University Press, Cambridge, 2000.

[79] R. Luchsinger and C. Grab, "Vertex reconstruction by means of the method of Kalman filter", Comput. Phys. Commun. 76 (1993) 263-280.

[80] P. Avery, "Applied fitting theory I: General least squares theory". CLEO Note CBX 91-72, October, 1991.

[81] P. Avery, "Applied fitting theory VI: Formulas for kinematic fitting". CLEO Note CBX 98-37, April, 1998.

[82] S. Choi. Private communication, March, 2001.

[83] CDF Collaboration, F. Abe et al., "Measurement of $B$ hadron lifetimes using $J / \psi$ final states at CDF", Phys. Rev. D57 (1998) 5382-5401.

[84] T. Sjöstrand, L. Lönnblad, and S. Mrenna, "PYTHIA 6.2: Physics and manual", Comput. Phys. Commun. 135 (2001) 238, arXiv:hep-ph/0108264.

[85] "QQ - The CLEO event generator". http://www. lns. cornell.edu/public/CLEO/soft/QQ (Unpublished).

[86] Application Software Group, "Detector description and simulation tool", CERN Program Library Long Writeup W5013, CERN, March, 1995.

[87] M. Narain. Private communication, February, 2001.

[88] K. Anikeev et al., "B Physics at the Tevatron: RunII and Beyond", 2002. FERMILAB-Pub-01/197. 
[89] R. Jesik, "Prospects for DØ beauty physics measurements in Run II". Talk given at IV International Conference on Hyperons, Charm and Beuty Hadrons, Valencia, Spain, June, 2000. (Published as [90]).

[90] R. Jesik, "Prospects for DØ beauty physics measurements in Run II", Nucl. Phys. Proc. Suppl. 93 (2001) 313-316.

[91] P. Ball et al., "B decays at the LHC", in Standard Model physics (and more) at the LHC, G. Altareli and M. L. Mangano, eds., CERN. 2000. arXiv:hep-ph/0003238.

[92] G. Cowan, Statistical Data Analysis. Oxford Science Publications, Oxford, 1998.

[93] R. Barlow, A Guide to the Use of Statistical Methods in the Physical Sciences. John Wiley \& Sons, Chichester, 1989.

[94] F. James, "MINUIT - Function minimization and error analysis", CERN Program Library Long Writeup D506, CERN, August, 1998. 